The undersigned hereby recommend to

The Faculty of Graduate Studies and Research

acceptance of the thesis,

\title{
Performance Optimization of the ORION Peer-to-Peer File Sharing Protocol for Mobile Ad Hoc Networks Using Unicast Messaging
}

submitted by

Ahmed Abada,

In partial fulfillment of the requirements for the degree of Master of Applied Science in Electrical Engineering

Prof. Changcheng Huang, Carleton University, Supervisor

Chairman, Department of System and Computer Engineering

Carleton University

August 2006 


$\begin{array}{ll}\begin{array}{l}\text { Library and } \\ \text { Archives Canada }\end{array} & \begin{array}{l}\text { Bibliothèque et } \\ \text { Archives Canada }\end{array} \\ \begin{array}{l}\text { Published Heritage } \\ \text { Branch }\end{array} & \begin{array}{l}\text { Direction du } \\ \text { Patrimoine de l'édition }\end{array} \\ \begin{array}{l}\text { 395 Wellington Street } \\ \text { Ottawa ON K1A ON4 }\end{array} & \begin{array}{l}\text { 395, rue Wellington } \\ \text { Ottawa ON K1A ON4 } \\ \text { Canada }\end{array}\end{array}$

Your file Votre référence ISBN: 978-0-494-18309-0 Our file Notre référence ISBN: 978-0-494-18309-0

NOTICE:

The author has granted a nonexclusive license allowing Library and Archives Canada to reproduce, publish, archive, preserve, conserve, communicate to the public by telecommunication or on the Internet, loan, distribute and sell theses worldwide, for commercial or noncommercial purposes, in microform, paper, electronic and/or any other formats.

The author retains copyright ownership and moral rights in this thesis. Neither the thesis nor substantial extracts from it may be printed or otherwise reproduced without the author's permission.
AVIS:

L'auteur a accordé une licence non exclusive permettant à la Bibliothèque et Archives Canada de reproduire, publier, archiver, sauvegarder, conserver, transmettre au public par télécommunication ou par l'Internet, prêter, distribuer et vendre des thèses partout dans le monde, à des fins commerciales ou autres, sur support microforme, papier, électronique et/ou autres formats.

L'auteur conserve la propriété du droit d'auteur et des droits moraux qui protège cette thèse. $\mathrm{Ni}$ la thèse ni des extraits substantiels de celle-ci ne doivent être imprimés ou autrement reproduits sans son autorisation.
In compliance with the Canadian

Privacy Act some supporting forms may have been removed from this thesis.

While these forms may be included in the document page count, their removal does not represent any loss of content from the thesis.
Conformément à la loi canadienne sur la protection de la vie privée, quelques formulaires secondaires ont été enlevés de cette thèse.

Bien que ces formulaires aient inclus dans la pagination, il n'y aura aucun contenu manquant.

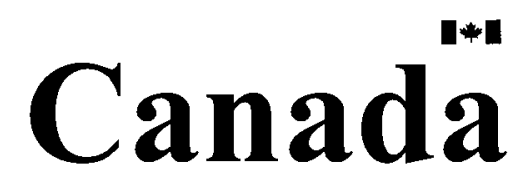




\title{
Performance Optimization of the ORION Peer-to-Peer File Sharing Protocol for Mobile Ad Hoc Networks Using Unicast Messaging
}

By

\author{
Ahmed Abada \\ B. Applied Science, University of Ottawa, 2002 \\ A thesis submitted to \\ The Faculty of Graduate Studies and Research \\ in partial fulfillment of \\ the requirements for the degree of
}

\section{Master of Applied Science in Electrical Engineering}

Ottawa-Carleton Institute for Electrical and Computer Engineering

Department of Systems and Computer Engineering

Carleton University

Ottawa, Ontario

CCopyright

2006, Ahmed Abada 


\begin{abstract}
Peer-to-Peer (P2P) file sharing protocols and Mobile Ad Hoc networks (MANETS) are both very important areas of research nowadays because of the constantly escalating demands from consumers to go mobile and the need to share contents between users. Because earlier P2P file sharing protocols designed for wireline networks did not perform adequately in MANETs [1], two new P2P file sharing protocols (MPP and ORION) were especially designed for the MANET environment. Both of MPP and ORION use either aggressive or reactive flooding techniques for their path selection and recovery. The aggressive techniques guarantees using the best path available in the network for file transfer but they require frequent network-wide flooding to find a new path each time a path failure happens, on the other hand, the reactive techniques do not require frequent flooding of the network but they run into the problem of not choosing the best available path because no mechanism exists to reorder the available paths after node movements. In this thesis we present a new extension to the ORION protocol $(\mathrm{ORION}+)$ that uses a new technique (Reactive flooding with unicast messaging) for its path selection and recovery. ORION+ avoids the need for frequent flooding associated with the aggressive technique by introducing a new unicast messaging mechanism to the reactive flooding technique by which it can probe its set of available paths for their response time and use the best one for the file transfer. Our new technique is based on unicast messaging which proves to be scalable and economic in terms of its bandwidth usage as per the results of our simulations.
\end{abstract}




\section{ACKNOWLEDGEMENT}

I would like to express my sincere thanks to my supervisor Professor Changcheng Huang for his support, patience and guidance throughout this project.

I would also like to express a very special thanks to my parents, and to my wife and daughter without their support and encouragement this work would not have been possible. 


\section{Table of Contents}

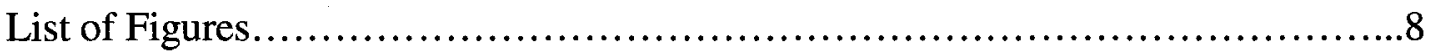

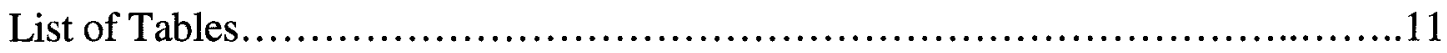

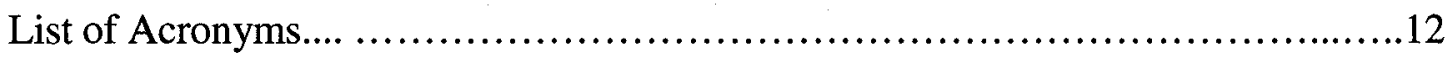

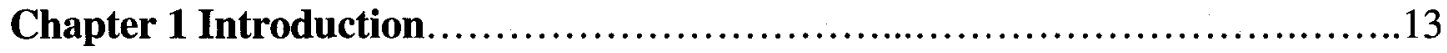

Chapter 2 Mobile Ad hoc Networks (MANETs)... .............................16

2.1 Characteristics of MANETs. ........................................ 16

2.2 Routing in MANETs... .............................................

2.2.1 Reactive (On-Demand) Routing .............................18

2.2.1.1 AODV. .............................................. 19

2.2.1.2 DSR .............................................20

2.2.2 Proactive (Table-driven) Routing Protocols.. ...................23

2.2.2.1 OLSR ...........................................23

2.2.2.2 DSDV.........................................25

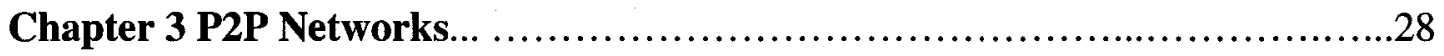

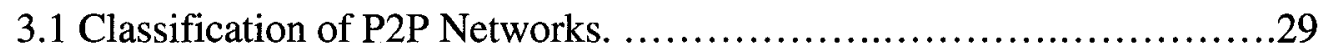

3.1.1 Centralized Networks............................................30

3.1.2 Decentralized (Pure P2P) Networks.. ..........................32

3.1.3 BitTorrent P2P System........................................

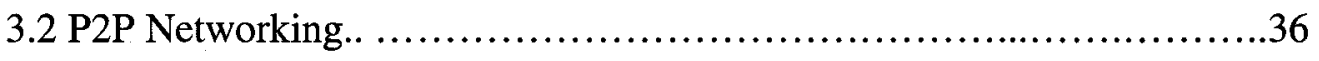

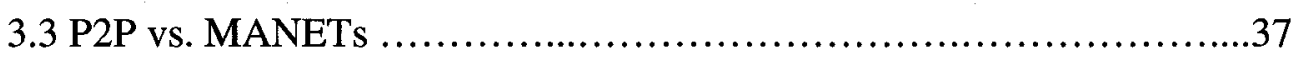

3.3.1 Differences between P2P and MANETs.......................38

3.3.2 Similarities between P2P and MANETs.......................39 
3.4 P2P over MANETs.... .........................................40

Chapter 4 File Sharing in P2P over MANETs............................41

4.1 The MPP P2P File Sharing Protocol.............................42

4.1.1 The MPP application layer protocol.......................43

4.1.2 The EDSR network layer protocol.......................44

4.1.3 The MPCP interlayer communication protocol..............45

4.1.4 Protocol Operation...................................45

4.1.5 Recovery from link failures.........................47

4.1.5.1 Static Downloading.........................47

4.1.5.2 Dynamic Downloading........................47

4.2 The ORION P2P File Sharing Protocol. .............................48

4.2.1 The ORION File Search Phase..............................49

4.2.2 The ORION File Transfer phase... .........................53

4.2.3 Route maintenance during the File Transfer phase...........54

4.2.4 Packet Scheduling and Loss recovery.........................56

4.3 Current Path Selection and Recovery Mechanisms Used in MPP

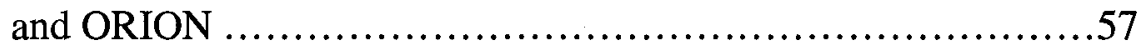

4.3.1 Aggressive Path Selection and Recovery..................58

4.3.2 Reactive Path Selection and recovery...................58

4.4 ORION vs. MPP................................................59

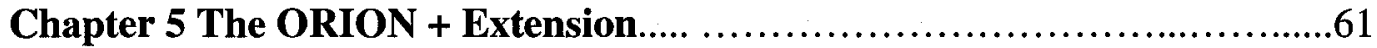

5.1 Possible Improvements for the ORION protocol..................63

5.1.1 BitTorrent alike downloading........................63 
5.2 The ORION+ Approach.........................................64

5.3 The ORION+ Implementation details............................69

5.4 Advantages and Disadvantages of ORION+ .....................74

5.4.1 Advantages..........................................74

5.4 .2 Disadvantages....................................75

5.4.2.1 The existence of only one source node in the network..................................... 75

5.4.2.2 No change in the quality of the available paths.......................................76

Chapter 6 Simulation and Results... ................................... 77

6.1 Simulation Model.. ......................................77

6.2 Simulation Setup.. .........................................79

6.2.1 Transfer time.. ........................................ 80

6.2.2 Successful file transfer.. ...........................90

6.2.3 Transmitted data volume..............................100

Chapter 7 Conclusion and Future Work................................ 110

References........................................................ 112 


\section{List of Figures}

Figure 2.1 Propagation of Route Request (RREQ) Packet.......................... 20

Figure 2.2 Path of Route reply (RREP) packet.............................. 20

Figure 2.3 Building route record during route discovery $\ldots \ldots \ldots \ldots \ldots \ldots \ldots \ldots \ldots \ldots \ldots . \ldots \ldots 22$

Figure 2.4 Propagation of route reply with route record........................... 22

Figure 2.5 Only MPRs of node A (B, D and F) need to retransmit to reach all

2 -hop neighbors................................................... 25

Figure 3.1 Physical topology Vs. Logical topology in P2P overlay networks............. 28

Figure 3.2 Centralized P2P network configuration................................ 30

Figure 3.3 The Napster scenario for providing a distributed file system for music

Files............................................................. 31

Figure 3.4 Gnutella decentralized P2P approach................................. 33

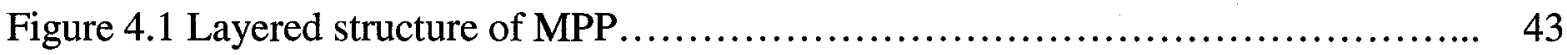

Figure 4.2 Message sequence chart for data search and download in MPP.............. 47

Figure 4.3 Node A floods a QUERY message for files 1, 2, 3 and $4 \ldots \ldots \ldots \ldots \ldots \ldots \ldots \ldots . .51$

Figure 4.4 Node B sends RESPONSE message with identifiers of local files............ 52

Figure 4.5 Node $\mathrm{C}$ sends RESPONSE message back to node B...................... 53

Figure 4.6 Node D sends RESPONSE message back to node B...................... 54

Figure 5.1 Tradeoff between different path selection and recovery mechanisms......... 65

Figure 5.2 File routing tables after node A initiated a file search for file " $\mathrm{X}$ " $\ldots \ldots \ldots \ldots . . .67$

Figure 5.3 Network after change in link quality............................... 69

Figure 5.4 File routing tables are not changed after change in the link quality........... 70 
Figure 5.5 Propagation of the LINK_QUALITY_REQ message to all available

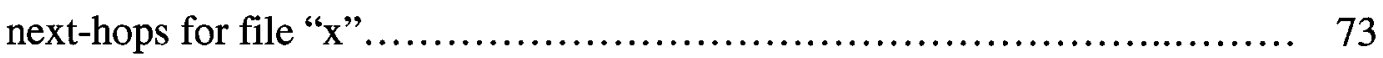

Figure 5.6 LINK_QUALITY_REPLY is sent in reply to LINK_QUALITY_REQ........ 74

Figure 5.7 File routing tables after node A initiated a file search for file " $\mathrm{X}$ "............ 76

Figure 5.8 No possible improvement if the requested file is only available once ......... 77

Figure 6.1 Transfer Delay vs. Node Mobility for 50 nodes and $2 \mathrm{~KB}$ file size........... 81

Figure 6.2 Transfer Delay vs. Node Mobility for 50 nodes and $50 \mathrm{~KB}$ file size.......... 81

Figure 6.3 Transfer Delay vs. Node Mobility for 50 nodes and $500 \mathrm{~KB}$ file size......... 82

Figure 6.4 Transfer Delay vs. Node Mobility for 50 nodes and $1 \mathrm{MB}$ file size........... 82

Figure 6.5 Transfer Delay vs. Node Mobility for 50 nodes and 3 MB file size........... 83

Figure 6.6 Transfer Delay vs. Node Mobility for 50 nodes and $10 \mathrm{MB}$ file size......... 83

Figure 6.7 Transfer Delay vs. Node Mobility for 50 nodes and 50 MB file size......... 84

Figure 6.8 Transfer Delay vs. Node Mobility for 50 nodes and $100 \mathrm{MB}$ file size......... 84

Figure 6.9 Transfer Delay vs. Node Mobility for 100 nodes and 2 KB file size.......... 85

Figure 6.10 Transfer Delay vs. Node Mobility for 100 nodes and $50 \mathrm{~KB}$ file size........ 85

Figure 6.11 Transfer Delay vs. Node Mobility for 100 nodes and $500 \mathrm{~KB}$ file size...... 86

Figure 6.12 Transfer Delay vs. Node Mobility for 100 nodes and 1 MB file size......... 86

Figure 6.13 Transfer Delay vs. Node Mobility for 100 nodes and 3 MB file size......... 87

Figure 6.14 Transfer Delay vs. Node Mobility for 100 nodes and 10 MB file size....... 87

Figure 6.15 Transfer Delay vs. Node Mobility for 100 nodes and 50 MB file size....... 88

Figure 6.16 Transfer Delay vs. Node Mobility for 100 nodes and 100 MB file size....... 98

Figure 6.17 Successful Transfer vs. Node Mobility for 50 nodes and 2 KB file size...... 90

Figure 6.18 Successful Transfer vs. Node Mobility for 50 nodes and $50 \mathrm{~KB}$ file size..... 90 
Figure 6.19 Successful Transfer vs. Node Mobility for 50 nodes and $500 \mathrm{~KB}$ file size....

Figure 6.20 Successful Transfer vs. Node Mobility for 50 nodes and 1 MB file size..... 91

Figure 6.21 Successful Transfer vs. Node Mobility for 50 nodes and 3 MB file size..... 92

Figure 6.22 Successful Transfer vs. Node Mobility for 50 nodes and $10 \mathrm{MB}$ file size.... 92

Figure 6.23 Successful Transfer vs. Node Mobility for 50 nodes and $50 \mathrm{MB}$ file size.... 93

Figure 6.24 Successful Transfer vs. Node Mobility for 50 nodes and $100 \mathrm{MB}$ file size... 93

Figure 6.25 Successful Transfer vs. Node Mobility for 100 nodes and $2 \mathrm{~KB}$ file size...... 94

Figure 6.26 Successful Transfer vs. Node Mobility for 100 nodes and 50 KB file size... 94

Figure 6.27 Successful Transfer vs. Node Mobility for 100 nodes and $500 \mathrm{~KB}$ file size... 95

Figure 6.28 Successful Transfer vs. Node Mobility for 100 nodes and 1 MB file size..... 95

Figure 6.29 Successful Transfer vs. Node Mobility for 100 nodes and 3 MB file size.... 96

Figure 6.30 Successful Transfer vs. Node Mobility for 100 nodes and 10 MB file size... 96

Figure 6.31 Successful Transfer vs. Node Mobility for 100 nodes and 50 MB file size... 97

Figure 6.32 Successful Transfer vs. Node Mobility for 100 nodes and 100 MB file size.. $\quad 97$

Figure 6.33 Data volume vs. Node Mobility for 50 nodes and $2 \mathrm{~KB}$ file size............ 99

Figure 6.34 Data volume vs. Node Mobility for 50 nodes and $50 \mathrm{~KB}$ file size......... 100

Figure 6.35 Data volume vs. Node Mobility for 50 nodes and $500 \mathrm{~KB}$ file size......... 100

Figure 6.36 Data volume vs. Node Mobility for 50 nodes and $1 \mathrm{MB}$ file size............ 101

Figure 6.37 Data volume vs. Node Mobility for 50 nodes and $3 \mathrm{MB}$ file size............ 101

Figure 6.38 Data volume vs. Node Mobility for 50 nodes and $10 \mathrm{MB}$ file size.......... 102

Figure 6.39 Data volume vs. Node Mobility for 50 nodes and 50 MB file size.......... 102

Figure 6.40 Data volume vs. Node Mobility for 50 nodes and 100 MB file size.......... 103

Figure 6.41 Data volume vs. Node Mobility for 100 nodes and $2 \mathrm{~KB}$ file size.......... 103 
Figure 6.42 Data volume vs. Node Mobility for 100 nodes and $50 \mathrm{~KB}$ file size......... 104

Figure 6.43 Data volume vs. Node Mobility for 100 nodes and $500 \mathrm{~KB}$ file size......... 104

Figure 6.44 Data volume vs. Node Mobility for 100 nodes and $1 \mathrm{MB}$ file size........... 105

Figure 6.45 Data volume vs. Node Mobility for 100 nodes and $3 \mathrm{MB}$ file size.......... 105

Figure 6.46 Data volume vs. Node Mobility for 100 nodes and $10 \mathrm{MB}$ file size......... 106

Figure 6.47 Data volume vs. Node Mobility for 100 nodes and 50 MB file size......... 106

Figure 6.48 Data volume vs. Node Mobility for 100 nodes and $100 \mathrm{MB}$ file size........ 107

\section{List of Tables}

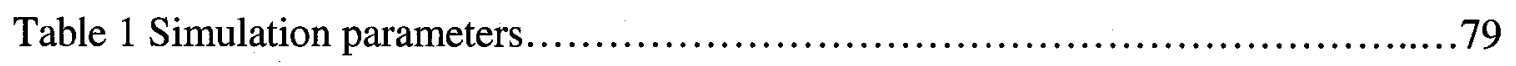




\section{List of Acronyms}

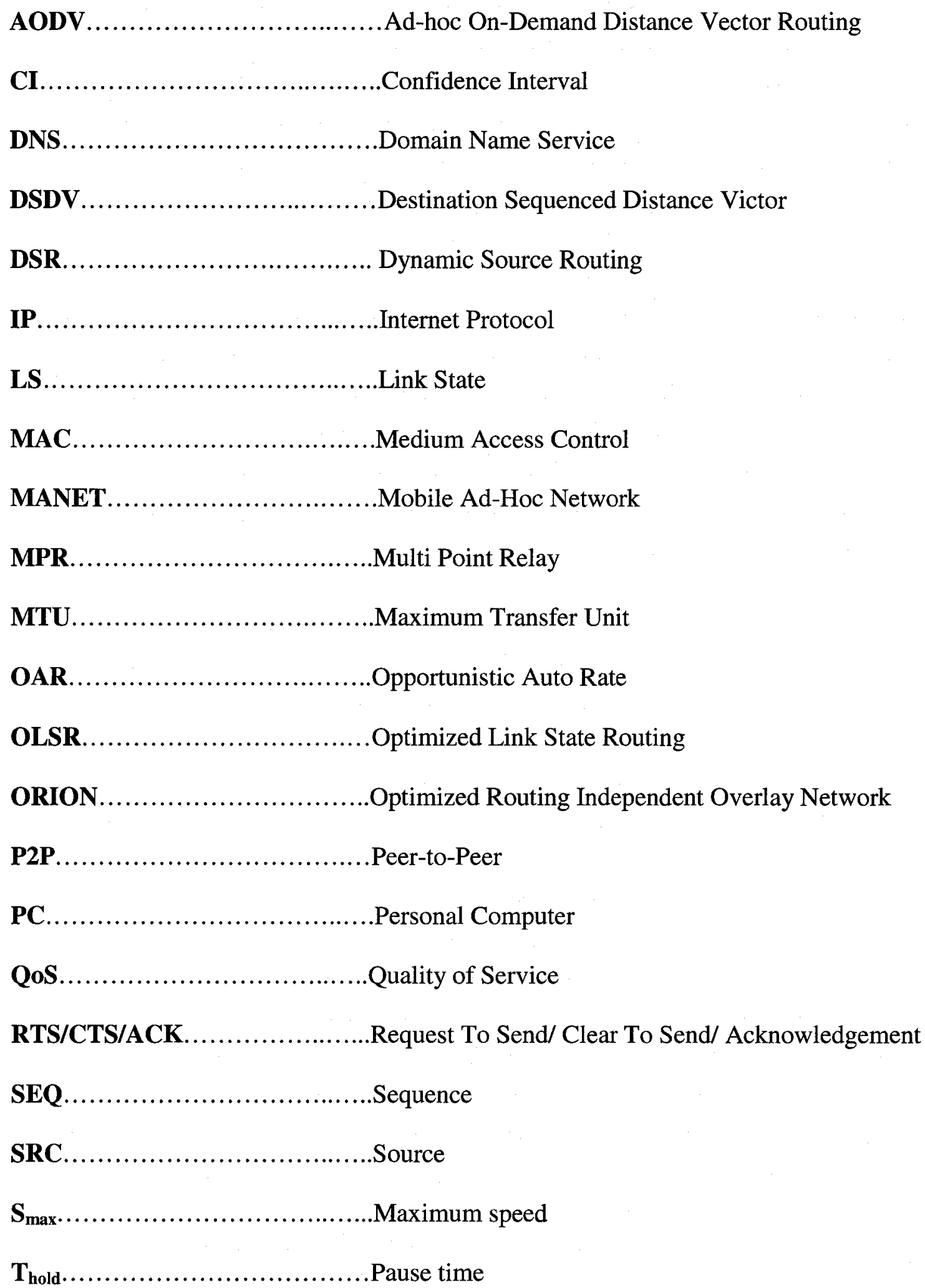




\section{Chapter 1 Introduction}

Mobile hosts and wireless networking hardware are becoming widely available, and extensive work has been done recently in integrating these elements into traditional networks such as the Internet. Often times, however, mobile users will want to communicate in situations in which no fixed wired infrastructure such as this is available, either because it may not be economically practical or physically possible to provide the necessary infrastructure or because the expediency of the situation does not permit its installation. For example, a class of students may need to interact during a lecture, friends or business associates may run into each other in an airport terminal and wish to share files, or a group of emergency rescue workers may need to be quickly deployed after an earthquake or flood. In such situations, a collection of mobile hosts with wireless network interfaces may form a temporary network without the aid of any established infrastructure or centralized administration. This type of wireless network is known as an ad hoc network [2].

A Mobile Ad Hoc Network (MANET) is a temporary self-organizing network of wireless mobile nodes without the support of any existing infrastructure that may be readily available on the conventional networks. They are usually used to provide quick connectivity between cooperating nodes in certain situations like battlefields, conferences and disaster relief environments. As the hardware development of today's mobile nodes is rapidly moving forward, they are becoming more powerful and are able to run applications that were originally designed for the desktop computers. One of the most important types of desktop applications today is the Peer-to-Peer File-Sharing type of 
applications. Like most of the popular desktop applications, Peer-to-Peer File-Sharing applications need to be adjusted to run on mobile nodes. Many Peer-to-Peer File-Sharing protocols already exist, but they were mainly designed for the conventional wireline networks and they lack any support for MANETs or wireless connectivity. Recently, some good work has been done in this field to adapt the Peer-to-Peer File-Sharing applications for running on mobile nodes. Two main protocols were developed in this area; they are the ORION protocol [1] and the MPP protocol [3] [9].

The ORION file-sharing protocol consists of two phases, the "File Search" phase and the "File Transfer" phase. During the "File Search" phase, a node searching for a file floods a QUERY message that contains some information about the file it is looking for throughout the P2P overlay network. A neighboring node that have the required file will respond to the searching node with a REPLY message containing the routing information that tells the searching node how to get to it. After receiving at least one REPLY message from responding nodes, the searching node can begin the "File Transfer" phase by sending a FILE_REQ message to the first node that responded with a REPLY message. After receiving a FILE_REQ message, the node that have the requested file can begin sending it to the requesting node.

During the "Query" phase, routes extracted from the REPLY messages are ordered on a first-come first-serve basis without considering the link quality of each route. While it is generally safe to assume that nodes with better link quality will be faster to send back a REPLY message and therefore be at the top of the list, however the link 
quality of each route may change over the course of the "File Transfer" phase because of node movement or a drop in the battery level resulting in weaker links being used while better ones are not even tested. The current ORION protocol allows the requesting node to switch to an alternative route only if the current route being used is down and not if there is a better route that can provide the same file.

In this thesis, we introduce an extension to the ORION protocol called ORION+. The main purpose of this extension is to test periodically all available routes acquired during the file search phase of the ORION protocol and select the best route among them to reduce the download time and provide a better overall user experience. We also present our simulation results that prove such improvement on the protocol can be achieved with a very low cost. 


\section{Chapter 2 Mobile Ad hoc Networks (MANETs)}

Mobile ad hoc networks are usually autonomous systems of mobile hosts (also serving as routers) connected by wireless links. In MANETS, mobile nodes communicate with each other using multi-hop wireless links. There is no stationary infrastructure; for instance, there are no base stations. Each node in the network also acts as a router, forwarding data packets for other nodes. A central challenge in the design of ad hoc networks is the development of dynamic routing protocols that can efficiently find routes between two communicating nodes $[4][5][6]$.

\subsection{Characteristics of MANETs}

\section{- Mobile}

The nodes are free to move around resulting in a dynamic network topology.

\section{- Wireless}

MANET uses shared wireless medium (radio, 802.11, infrared, etc.) to transmit and receive data.

\section{- Self-organizing, distributed, and infrastructure-less}

They are self-organizing in nature. There is no centralized control, which implies that network management will have to be distributed across various nodes. 
- Multi-hop

A message from source node to destination node goes through multiple nodes because of limited transmission radius. Every node acts as a router and forwards packets from other nodes to facilitate multi-hop routing.

\section{- Scarce resources}

The wireless links have limited bandwidth and they are also error prone. In addition, the mobile nodes have limited battery and processing power. So, energy is a scare resource.

\section{- Temporary and rapidly deployable}

These networks are temporary in nature. There is no base station. Whenever the nodes are within their transmission radius, they form an ad hoc network. Hence, they are rapidly deployable.

- Neighborhood awareness

Host connections in MANETs are based on geographical distance.

\subsection{Routing in MANETs}

Regarding MANETs, we distinguish two basic routing categories, i.e., proactive and reactive routing approaches. Proactive protocols periodically maintain the routing information so that any node can use existing routes to reach destinations at any time. 
This is the rule in fixed networks, but in mobile ad hoc networks this would require a very frequent update of routing information for all nodes, which implies a lot of overhead. Reactive protocols, on the other hand, obtain the necessary routing information only when a route is needed between two nodes; the route is maintained only when the route is active. This is why reactive protocols are called "on-demand" protocols. Reactive protocols imply lower overhead than proactive ones, but they suffer from route setup delays when a communication flow is to start. To illustrate on the functionality of MANET routing protocols, we next introduce briefly two protocols from each routing category [7] [8] [9] [10].

\subsubsection{Reactive (On-Demand) Routing}

In reactive routing schemes, the route between two nodes in the network is determined on-demand only. They do not need to exchange control data in absence of data traffic. Only in case a reactive routing protocol wants to set up a route to another node, it floods a route request through the network. In case of success, a response message is sent back either from the destination or an intermediate node, which knows the route to the destination by a formerly made route request. Examples of reactive routing schemes are the Ad-hoc On-Demand Distance Vector Routing (AODV) and the Dynamic Source Routing (DSR). 


\subsubsection{AODV}

Route Requests (RREQs) and Route Replies (RREPs) are the two message types defined by AODV. When a route to a new destination is needed, the node uses a broadcast RREQ to find a route to the destination (Figure 2.1). A route can be determined when the request reaches either the destination itself or an intermediate node with a fresh route to the destination. The route is made available by unicasting a RREP back to the source of the RREQ (Figure 2.2). Since each node receiving the request keeps track of a route back to the source of the request, the RREP Reply can be unicast back from the destination to the source, or from any intermediate node that is able to satisfy the request back to the source [5].

A hello message is a local advertisement for the continued presence of the node. Neighbors that are using routes through the broadcasting node will continue to mark the routes as valid. If hello messages from a particular node stop coming, the neighbor can assume that the node has moved away. When that happens, the neighbor will mark the link to the node as broken and may trigger a notification to some of its neighbors telling that the link is broken.

In AODV, each router maintains route table entries with the destination IP address, destination sequence number, hop count, next hop ID and lifetime. This information must be kept even for ephemeral routes, such as those created to temporarily keep track of reverse paths towards nodes originating the RREQs [11]. 


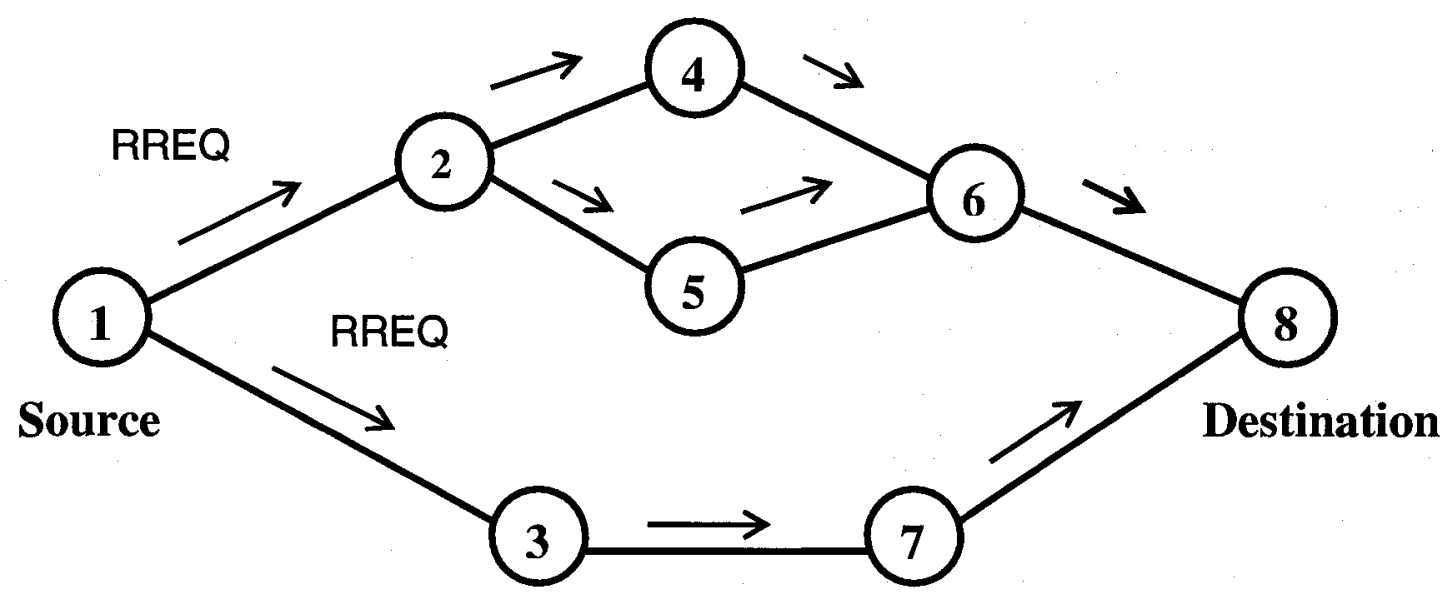

Figure 2.1 Propagation of Route Request (RREQ) Packet

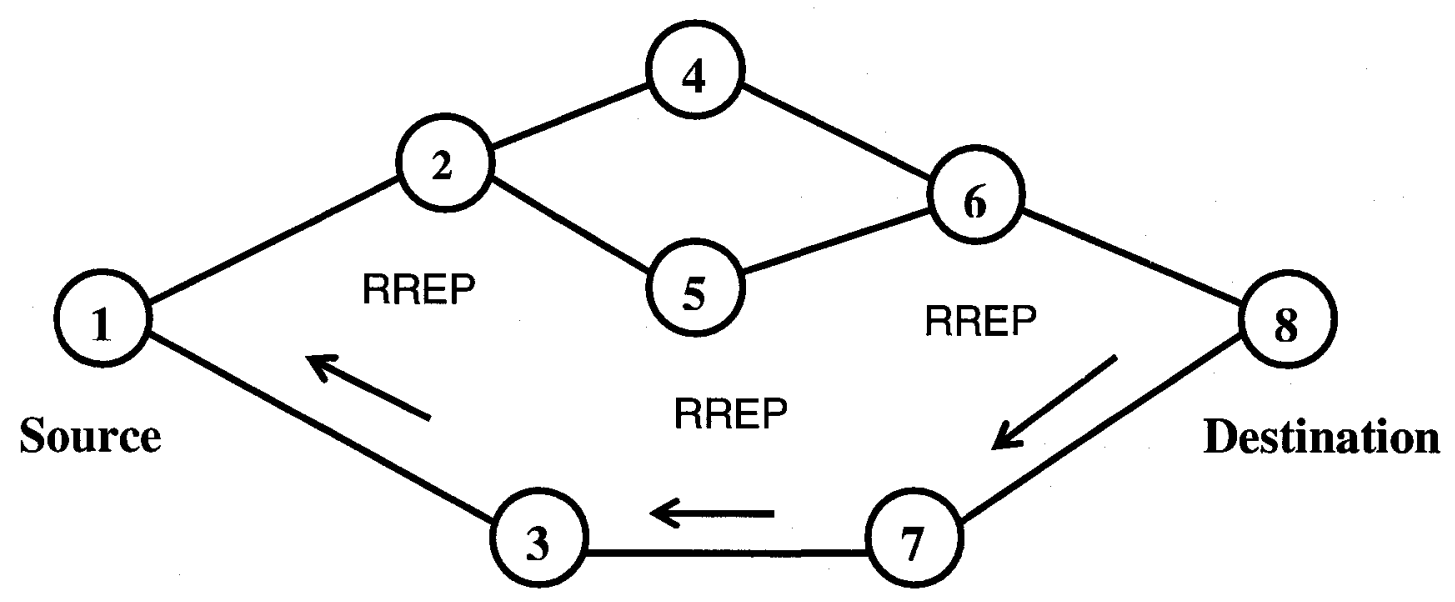

Figure 2.2 Path of Route reply (RREP) packet

\subsubsection{DSR}

DSR is based on the idea of source routing where intermediate nodes are not required to keep routing information to route packets from a source to a destination. Instead, each data packet must carry the complete routing information. Nodes in DSR are required to maintain source-route caches of known routes to other nodes in the network. 
Source-route caches are used to send own data packets or to respond to other nodes route requests.

When a source node wants to send a data packet to a destination node that it does not have a route to, it must initiate a route discovery process. During the route discovery, the source node floods the network with a RREQ message that contains the destination node address, the source node address and a unique identification number (Figure 2.3). Intermediate nodes receiving the RREQ for the first time will either cast a RREP back to the source if they have a cached route to the required destination (Figure 2.4) or they will append their own addresses to the list of nodes traversed so far by the message and rebroadcast it to their own neighbors otherwise.

DSR does not require symmetric links to operate. When the RREQ message reaches the destination or an intermediate node with a cached route to the destination, if symmetric links are supported, the route carried by the RREQ will be just reversed and used as the source route from the destination node to the source node to route the RREP message, on the other hand, if symmetric links are not supported, the destination node will have to do its own route discovery to the source node and piggyback the extracted source route on the new RREQ message.

In DSR, nodes are allowed to cache in source routes learned from overheard RREP packets. When link failure happens at an intermediate node and data cannot be forwarded to the next hop, a RERR packet is unicasted to the source node in order to 
inform it about the link failure. After receiving the RERR, the source node must discard the erroneous route and either use another cached route to the same destination if it have one, or initiate a new route discovery process to get a new route.

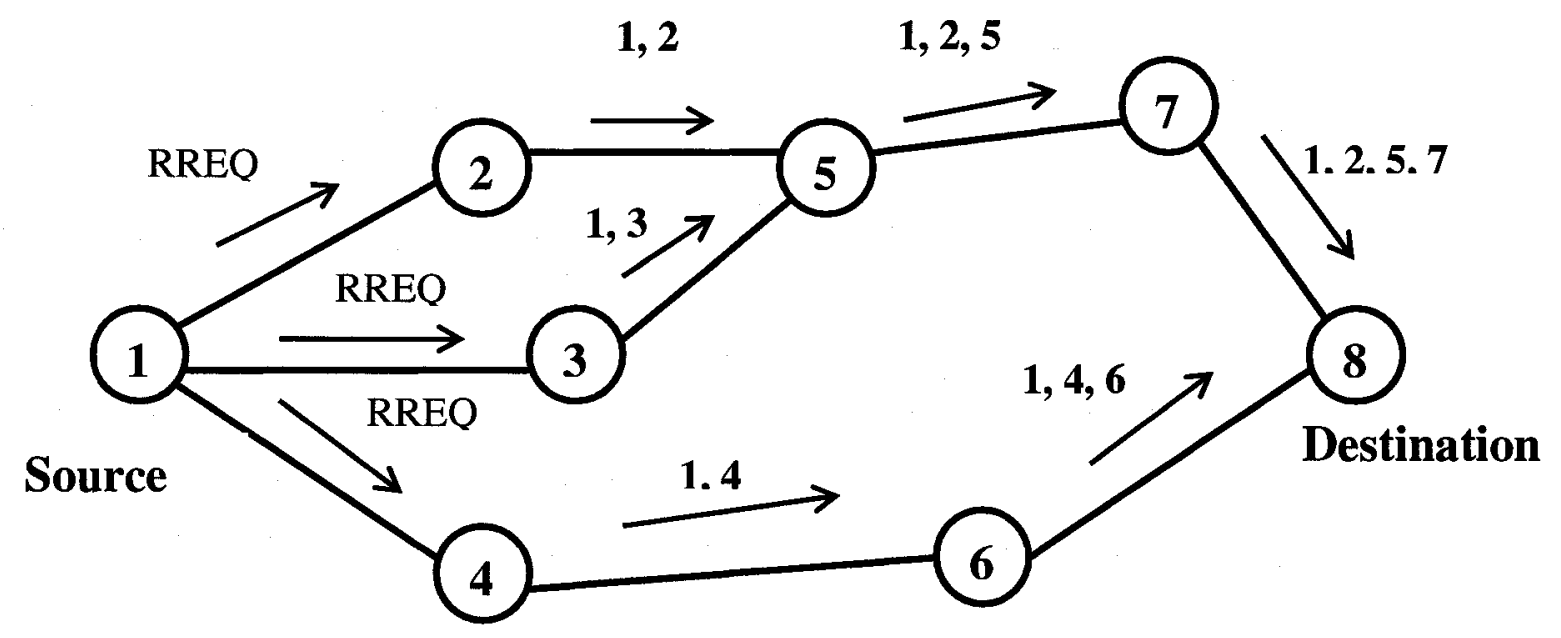

Figure 2.3 Building route record during route discovery

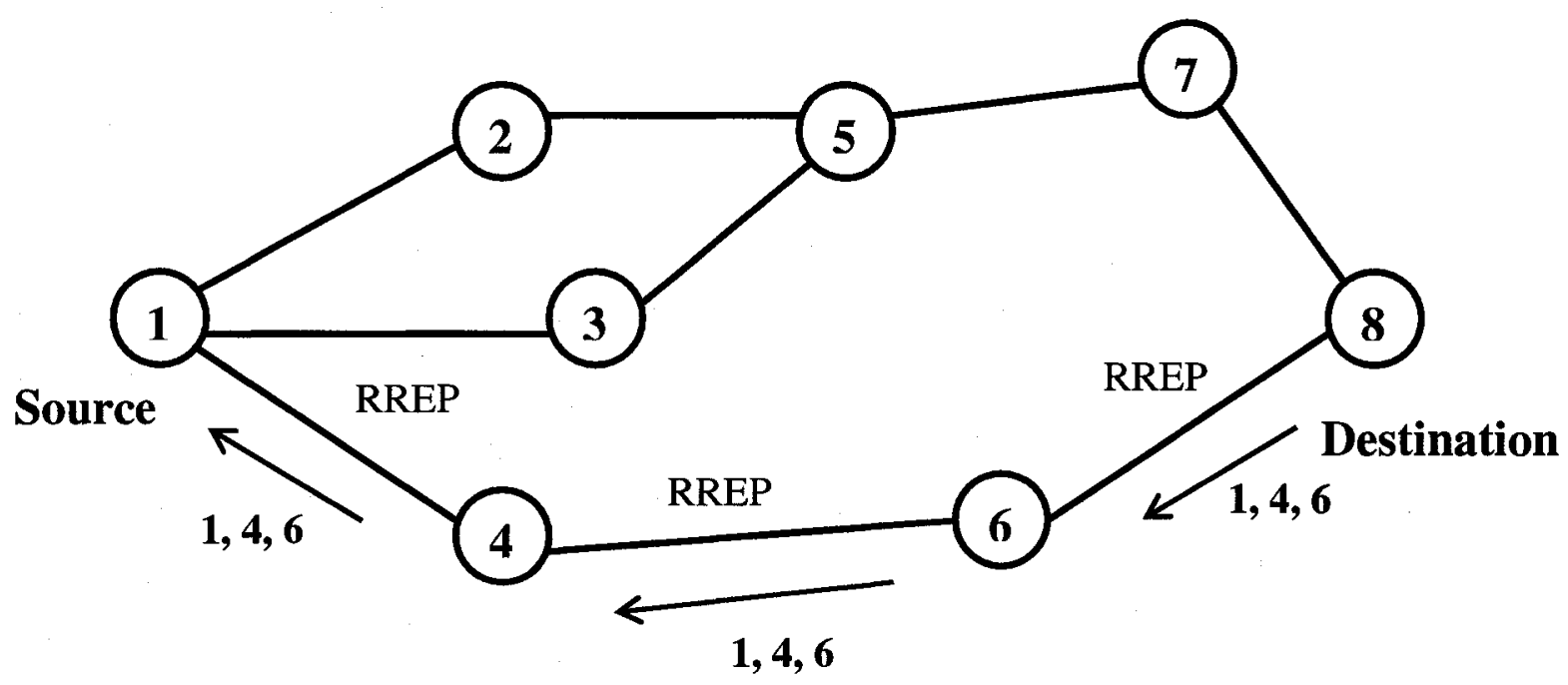

Figure 2.4 Propagation of route reply with route record 


\subsubsection{Proactive Routing Protocols}

The main characteristic of a proactive routing protocol is to maintain consistent, up-to-date routing information among all nodes in the network. For this purpose, each node must maintain one or more tables to store routing information. Thus the route establishment can take place very fast. The disadvantage of this class of routing protocols is the necessity for periodic updates of the network topology. They generate additional traffic, which wastes network bandwidth and battery power.

Proactive routing protocols share a common feature, that is, background routing information exchange regardless of communication requests. The protocols can provide low-latency route access, which is a very desirable property especially for applications using real-time traffic. Many proactive routing protocols have been proposed for efficiency and scalability. Examples of proactive routing protocols are Optimized Link State Routing Protocol (OLSR), and Destination-Sequenced Distance-Vector Routing (DSDV) [3].

\subsubsection{OLSR}

Optimized Link State Routing Protocol (OLSR) is a LS protocol. It periodically exchanges topology information with other nodes in the network. The protocol uses MultiPoint Relays (MPRs) to reduce the number of broadcast packet retransmissions and also the size of the LS update packets, leading to efficient flooding of control messages in the network. 
OLSR nodes periodically broadcast HELLO messages to all of their immediate neighbors to exchange neighborhood information (i.e., list of neighbors) and to compute the MPR set. From neighbor lists, nodes can figure out the nodes that are two hops away and computes the minimum set of one-hop relay points required to reach all two-hop neighbors. Such set is the MPR set. Figure 2.5 illustrates the MPR set of node A (nodes B, D and F), here we can see that node A can reach all its two hop neighbors via its MPR set. Each node informs its neighbors about its MPR set in the HELLO message. Upon receiving such a HELLO, each node records the nodes (called MPR selectors) that select it as one of their MPRs. In routing information dissemination, OLSR differs from pure LS protocols in two aspects. First, by construction, only the MPR nodes of node A need to forward the link state updates issued by A. Second, the link state update of node A is reduced in size since it includes only the neighbors that select node $\mathrm{A}$ as one of their MPR nodes. In this way, partial topology information is propagated, that is, say; node A can be reached only from its MPR selectors. OLSR computes the shortest path to an arbitrary destination using the topology map consisting of all of its neighbors and of the MPRs of all other nodes. OLSR is particularly suited for dense networks. When the network is sparse, every neighbor of a node becomes a multipoint relay. The OLSR then reduces to a pure LS protocol [12]. 


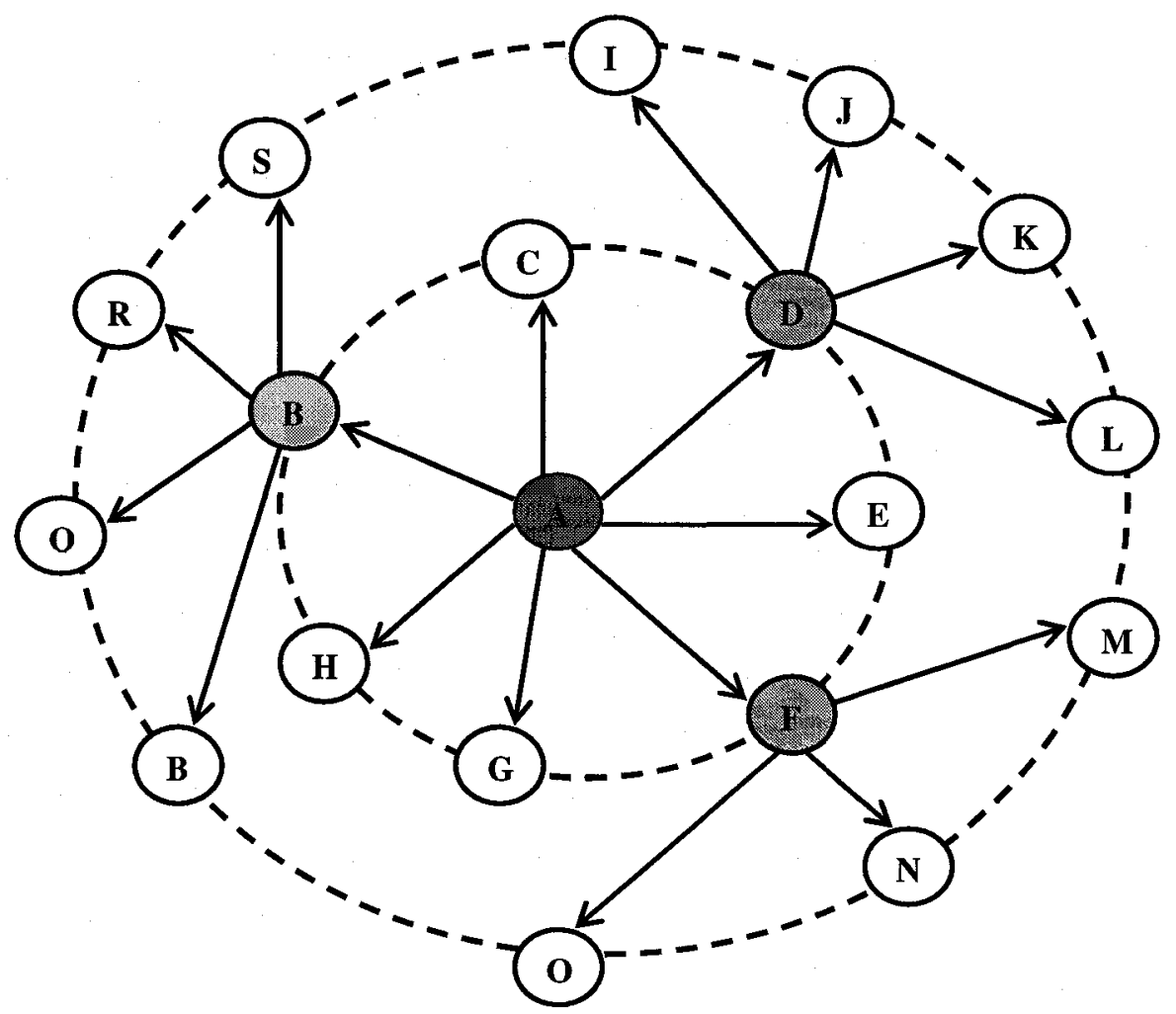

Figure 2.5 Only MPRs of node A (B, D and F) need to retransmit to reach all 2-hop neighbors

\subsubsection{DSDV}

DSDV is a hop-counting distance vector protocol requiring each node to periodically broadcast routing updates. In DSDV, each node maintains a routing table that has an entry for each destination in the network. The attributes for each destination are the next hop ID, hop count metric and a sequence number, which is originated by the destination node. DSDV uses both periodic and triggered routing updates. Triggered routing updates are used in addition to the periodic updates in order to propagate the routing information as quickly as possible whenever there is any topological change. The 
update packets include the destinations accessible from each node and the number of hops required to reach each destination along with the sequence number associated with each route.

Upon receiving a route update packet, each node compares it to the existing information regarding the route. Routes with old sequence numbers are simply discarded. In case of routes with equal sequence numbers, the recently advertised route replaces the old one if it has a better hop count metric. The metric is then incremented by one, since the incoming packet will require one more hop to reach the destination. Newly recorded routes are immediately advertised to the neighbors.

When a link to a next hop is broken, any route through that next hop is immediately assigned an infinite value with a new sequence number. When a node receives an infinite count and has an equal or greater sequence number with a finite metric, a route update broadcast is triggered. Thus, real routes propagated from the newly located destination will quickly replace the routes with infinite value.

DSDV also employs a mechanism to damp out fluctuations in route table updạtes. In an environment where many independent nodes transmit routing information asynchronously fluctuations could arise. For example, a node could receive two routes to the same destination with the same sequence number, but say the one with the worse metric always arrives first. This could lead to continuous outbursts of route updates. DSDV solves this problem by using "settling time" data. Specifically, time duration until 
the route becomes stable (termed settling time) is predicted, and the settling time is allowed before advertising any new route information to the network. In other words, the settling time is used to decide how long to wait before advertising new routes. Delaying the advertisement of unstable routes, fluctuations in the routing tables are prevented and thus the number of route updates is reduced.

The protocol has a number of drawbacks. Optimal values for the parameters like maximum settling time for a particular destination are difficult to determine. This might lead to route fluctuations and spurious advertisements resulting in waste of bandwidth. DSDV uses both periodic and triggered routing updates, which could also cause excessive communication overhead. In addition, in DSDV a node has to wait until it receives the next route update originated by the destination before it can update its routing table entry for that destination. Finally, DSDV does not support multi-path routing [4]. 


\section{Chapter 3 P2P Networks}

Peer-to-peer networks (P2P) have become a very popular medium of content distribution over the Internet in the past few years. In such networks, computer resources and services are exchanged directly between systems. Beginning with the popular Napster phenomenon in the late 1990s, the popularity of P2P has dramatically increased the volume of data transferred between Internet users. Recent studies concluded that the file sharing activity on $\mathrm{P} 2 \mathrm{P}$ networks accounts for up to $60 \%$ of the Internet traffic on any given service provider network. Figure 3.1 shows the difference between the P2P application level network and the actual physical connections provided by intermediate routers.

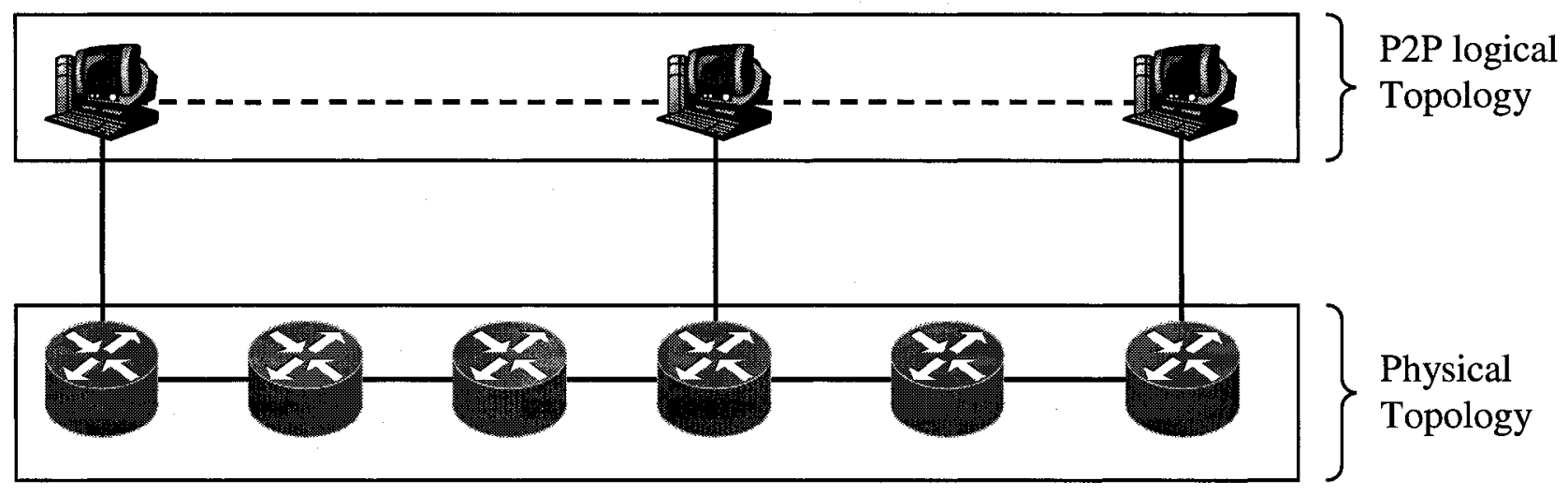

Figure 3.1 Physical topology vs. Logical topology in P2P overlay networks

P2P Networks provide an alternative mechanism to client/server paradigms for sharing resources. Existing P2P systems have been driving a major paradigm shift in the era of distributed computing. Many factors have fostered the recent explosive growth of such networks: low cost attributed to the fact that P2P networks may not require any 
high-performance centralized servers, high availability of large numbers of computing and storage resources and increased network connectivity.

\subsection{Classification of P2P Networks}

The nodes in P2P networks are termed as "servents" (or peers) since they act both as a client and as a server to the network at the same time. Content retrieval in P2P networks usually involves a content search phase and a content downloading phase. First, the initiator node searches for the desired content in the network. Then, after having discovered a servent sharing the requested content, it establishes a direct connection and transfers the resource from the provider.

Peer-to-peer networks can be broadly classified into two categories:

1. Centralized networks such as Napster.

2. Decentralized networks such as Gnutella.

In the former approach, there must be a central server that maintains file indexes to resources shared on the network and facilitates content search. In the decentralized approach, the entities directly discover each other by exchanging broadcast messages or by other mechanisms without involving any central server [13][10]. Another form of P2P networks that do not fall in any of the above categories is the BitTorrent P2P system [14] [15]. BitTorent nodes do not use any central server to search for content as in the centralized networks, neither they communicate randomly with neighboring nodes as in the decentralized networks. Because of the wide spread usage of the BitTorrent system nowadays, we are going to introduce it in section 3.1.3 as a special case of P2P systems. 


\subsubsection{Centralized Networks}

Such networks make use of a centralized indexing server that stores information about sharable files of each node in the P2P network. Figure 3.2 depicts the design of such networks and the steps involved in resource sharing. They are as the following:

Step 1: Each peer sends its location information on connecting to the network

Step 2: When client 1 intends to download a resource; it sends a search request to the central server

Step 3: The central server returns information about the peer (Client 2) sharing the resource

Step 4: Client 1 then connects directly with Client 2 and downloads the file

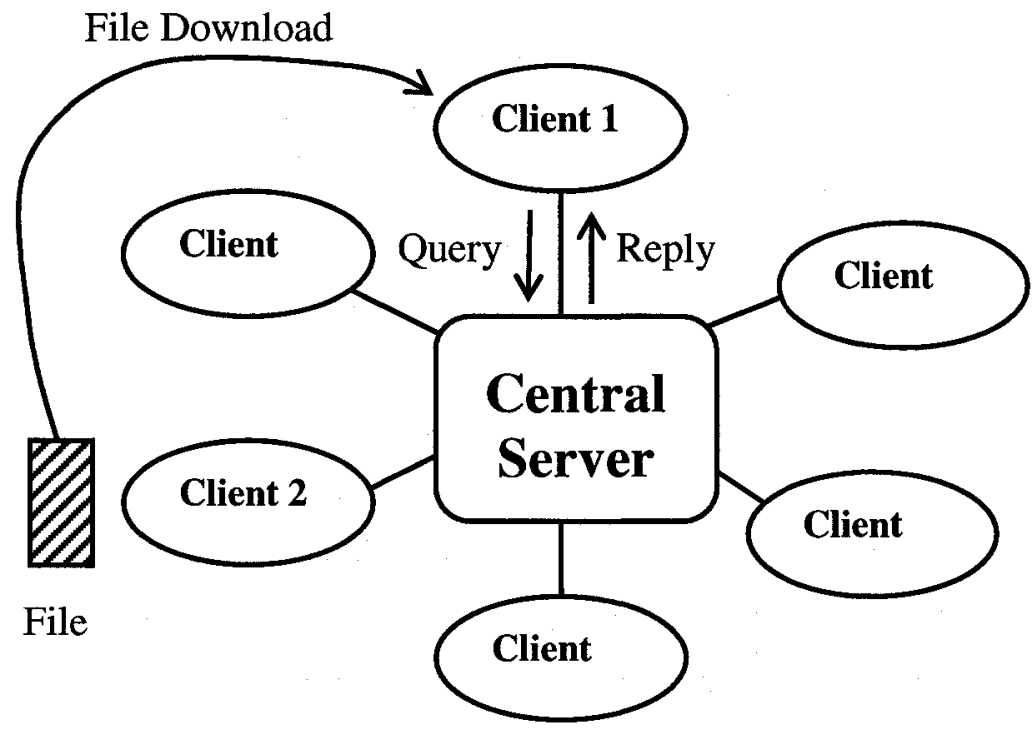

Figure 3.2 Centralized P2P network configuration 
This architecture offers very good performance in terms of the response time to search requests. However, such architectures may not scale well since the central server becomes a potential bottleneck. Further, due to the existence of a central server, these schemes are not robust to attacks - an attack on the central server can bring down the entire network. Napster is a very popular P2P network that uses centralized indexing. Napster collapsed due to litigations over potential copyright infringements.

\section{Example: File Sharing with Napster}

Napster, the famous MP3 file-sharing program, was launched in 1999. It had a revolutionary impact on the Internet due to its unique design, i.e. after the initial centralized Napster search; clients connected to each other and exchanged data directly from one system's disk to another. Figure 3.3 illustrates this process.

1. Construct database

- Users connect to the napster server

3. Server searches database. Finds song.mp3 on User B's machine

2. User A searches for song.mp3 3 3

User A

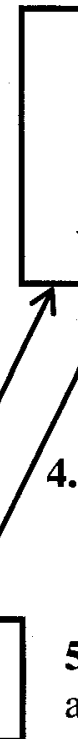

\section{www.napster.com} Central Server File List:

User B: song.mp3 User D: another.mp3

- Server builds up a list of available songs and locations

3

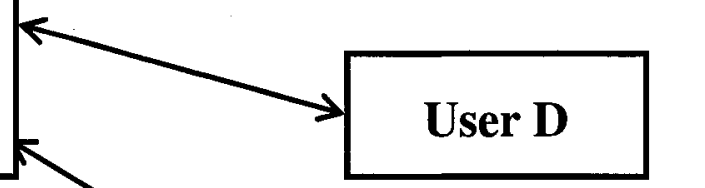

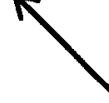

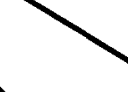


Users first connect to the main Naptser server and register themselves to join the network. The main server then obtains a list of all MP3's that the users are willing to share and adds them to the list of songs in its central database. When a user (User A) performs a search for a certain file, Napster searches its central database on the main server and then returns to the user, the address of the peer that has a copy of the file. (User A) then connects directly to the peer that has a copy of the file (User B) and downloads it directly without any further intervention from the main server unless communication is interrupted (because the peer has logged off for example).

Napster is P2P because the Napster peers bypass DNS and because once the Napster server resolves the IP address of the PCs hosting a particular song, it shifts control of the file transfers to the nodes.

\subsubsection{Decentralized (Pure P2P) Networks}

Decentralized networks adopt a distributed architecture for searching content shared by peers. Each peer discovers and establishes connections with a variable number of servents to exchange content. Peers discover each other in the P2P network using broadcast messages. When a certain node in the P2P network wants to search for a file, it broadcasts a search request for that file to the nodes directly connected to it. Each of these nodes in turn broadcasts the request to its neighbors, until the message reaches a peer who possesses the content or has traversed a maximum number of hops. Here, every peer is involved in handling and forwarding request messages, thus behaving both as a client and as a server at the same time. Gnutella is an example of a pure P2P network. 


\section{Example: File Sharing with Gnutella}

Gnutella is a 'true' P2P system because it does not rely on central control for lookup, organization or communication. A brief scenario is given in Figure 3.4.

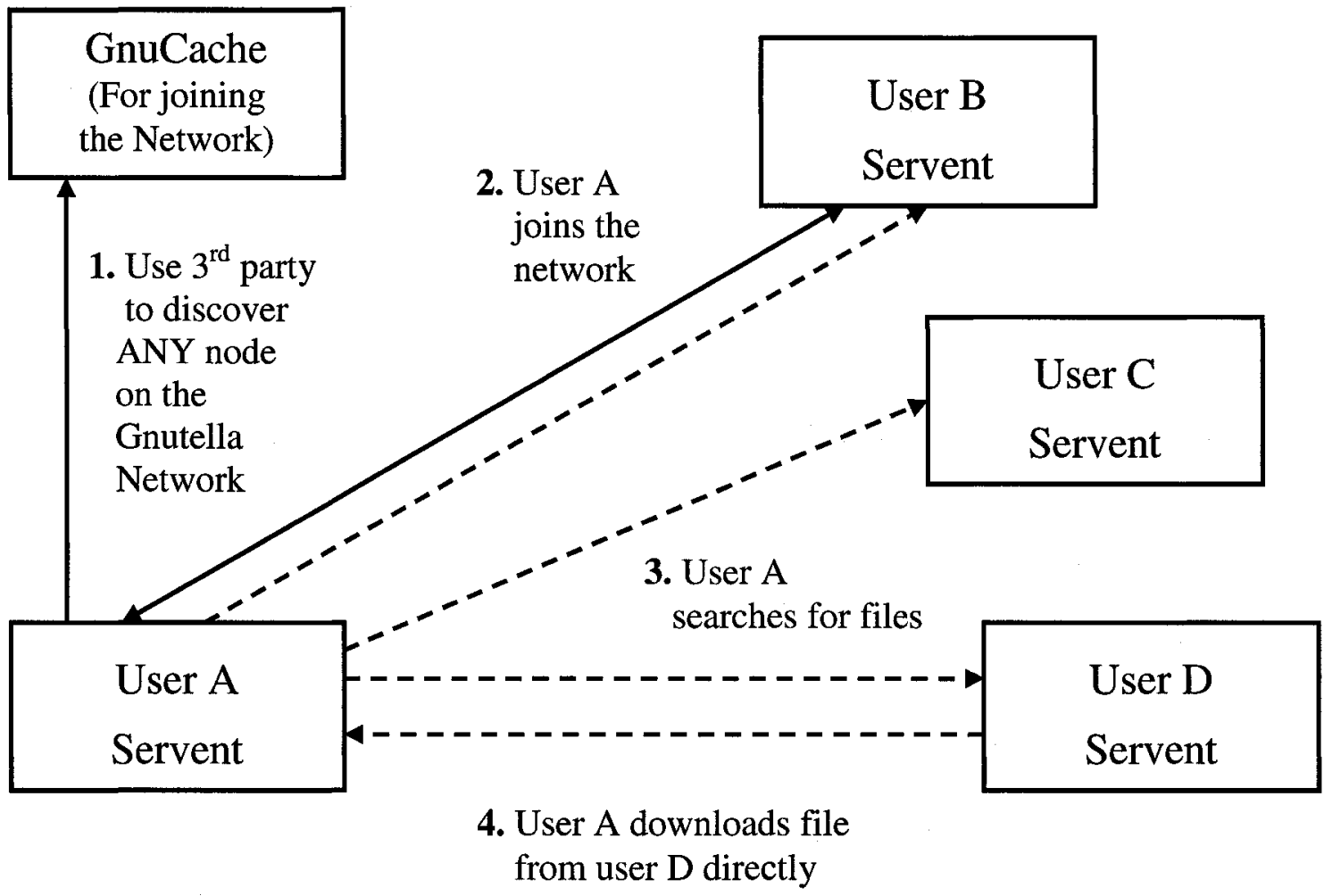

Figure 3.4 Gnutella decentralized P2P approach

There are several ways of joining a Gnutella network. The one given in Figure 3.4 uses a GnuCache as a lookup server for a list of Gnutella nodes but one could easily use other methods like Newsgroups or web sites to get lists of nodes currently online, so the GnuCache is not a single point of failure for the Gnutella system. Nodes join the network by connecting initially to any online Gnutella node. This can be any node in the network 
so it is generally easy to join in a decentralized fashion. After connecting to the Gnutella network, it is easy to discover other nodes through the first node. Gnutella nodes typically connect to 3 nodes and then search by broadcasting their search request to all connected neighbors. Each node repeats this search request to its neighbors and so on. In the example presented in Figure 3.4, User D has the required file so User A connects directly to User $\mathrm{D}$ and downloads the file using this point-to-point connection.

\subsubsection{BitTorrent P2P System}

BitTorrent is a P2P file distribution system that was designed for wireline networks. It enforces cooperation between nodes interested in downloading a certain file by making them exchange parts of the file with each other so that it is no longer the sole responsibility of a central serve to upload the whole file to all interested nodes. By doing so, the system achieves much faster downloading time and also makes use of the uploading capacity of each interested peer to support the distribution of the file. In addition to enforcing cooperation between downloading parties, BitTorrent also enables simultaneous Multi-Source downloading. In this scheme cooperating nodes are able to download from many sources simultaneously and hence cutting down further on the required downloading time.

For the BitTorent system to work, four different elements must exist. They are the following:

1) A*.torrent file

2) A web page to publish the *.torrent file on 
3) A tracker server to act as a rendezvous point so the nodes interested in a certain file can find each other

4) Nodes interested in downloading a file (Running a BitTorrent client)

In order to publish a file using BitTorrent, The file publisher will have to create a *.torrent file for each file he/she want to publish and post it on a web page. The *.torrent file contains all the necessary information about the shared file like its name, length, hashing information and the URL of the tracker server responsible for maintaining the information (keeping track) of which nodes are currently in the process of downloading the file or have a complete copy of the file. Trackers only help the nodes interested in downloading a certain file to find each other and do not take part in the downloading process (data exchange) in any way. After a certain node interested in downloading a certain file gets the *.torrent file of the file that it wants to download from the web page where the *.torent file is posted, it must contact the tracker server to obtain a list on nodes currently taking part in downloading the shared file. Tracker servers categorize nodes interested in a certain file to be either Seeds or Lechers. Seed nodes have a complete copy of the shared file while Lechers only have a subset of the total parts of the file. There ought to be at least one seed in the group of nodes returned by the tracker server to make sure that the group as a whole is not missing any part of the shared file. After getting the list of nodes (seeds and lechers) currently downloading the file from the tracker server, the BitTorrent client of the newly joining node must start the downloading process by contacting the corresponding BitTorrent clients of the other nodes currently downloading the file and start exchanging parts of the file with them (after getting the first part for 
free). BitTorrent does not use any central authority to coordinate the downloading process; each node is responsible to maximize its downloading rate by uploading only to cooperating nodes.

\subsection{P2P Networking}

P2P networking refers to a new distributed networking paradigm that employs application level overlay connections between participating nodes for the purpose of resource sharing between end systems. P2P systems were originally designed to run on stationary desktop computers connected to each other by the Internet over wireline physical connections for the purpose of file sharing. Due to the rapid advancement of wireless mobile computing devices in terms of processing, memory and battery power, there has been a growing demand to port the P2P file sharing applications to the wireless and MANET worlds.

The signaling traffic in $\mathrm{P} 2 \mathrm{P}$ networks consists mainly of network maintenance and content requests and responses. In pure P2P networks, network maintenance in this context means that the participants initiate at regular intervals "keep-alive" or neighborhood discovery messages to find the neighbors. Nodes receiving a neighborhood discovery message or a keep-alive request, reply with a keep-alive response. Thus every node knows at least a number of active participants in the overlay network, which are at least two hops in the overlay network away. To these nodes the node can connect if one of its direct neighbors fails. 
Further on, active peers issue in random intervals, determined by the user, content requests, to find the location of demanded content. As no knowledge about the topology of the network or the location of the content is available in pure P2P networks, these requests have to be flooded through the network. In contrast, in centralized P2P networks these requests are processed by the central server. Responses, $i$.e. keep-alive responses or content responses are mainly routed through the network on the same path the fastest query was transferred through the network.

In pure P2P networks, to be able to take part in the overlay P2P network, a new peer has to know at least one IP address of a node already participating in the network. Otherwise a new node cannot participate, as it is not able to establish any new connections in the overlay network. For the addresses of currently active nodes, a new node may either rely on cached addresses of nodes, which were active in a previous session, or it may contact a bootstrap server. The bootstrap server is a well known host with a stable IP-address, which may itself participate in the overlay network, or which simply caches the IP addresses of nodes which used the bootstrap server to enter the network in a kind of FIFO memory. As nodes which just connected to the network are assumed to stay connected further on, the bootstrap server can thus provide IP addresses of active nodes with a high probability without actively participating in the overlay network [16]. 


\subsection{P2P vs. MANETs [17]}

P2P networks and MANETs have several aspects in common since they are both based on the same idea, which is to provide connectivity in a completely decentralized manner. Yet they are also different because they were designed differently for different purposes, $\mathrm{P} 2 \mathrm{P}$ was designed to facilitate file sharing in wireline networks and MANETs were designed to establish network connectivity for mobile wireless nodes. In this section we briefly outline the main similarities and differences between P2P and MANETs.

\subsubsection{Differences between P2P and MANETs}

P2P and MANETs were initially designed to serve different purposes. MANETs were designed to provide network level connectivity to a specific end-host with a unique IP address. In contrast, P2P provide application level connectivity to an end-host that has a specific file or service. In MANETs, only one end-host is sought to establish a connection with, while in $\mathrm{P} 2 \mathrm{P}$, any end-host having the required file or service is a candidate to establish the connection with. So the connections provided by MANETs and P2P can be classified as host-driven and data-driven respectively.

Since P2P was initially designed for wireline networks, nodes are usually not mobile and they can exist anywhere around the globe. MANET nodes on the other hand are mobile and they must coexist within a relatively small geographical area so they can communicate with each other using wireless links. 
The wireline physical infrastructure in P2P networks provide more reliable links with orders of magnitude less in bit error rate than its wireless counterpart in MANETs. Packet losses in P2P are mainly caused by buffer overflows in intermediate routers as a result of network congestion, whereas in MANETs they are often caused by link breaks due to node movements or by bad channel conditions

The routing in $\mathrm{P} 2 \mathrm{P}$ systems is classified as "content-driven" since the connection establishment criterion is based on the availability of a certain required content. Any node offering the sought content is a candidate for the connection. On the other hand, routing in MANETs is classified as "user-driven" since the connection establishment criterion is a specific (with no substitute) IP address of a known user or end-system.

\subsubsection{Similarities between P2P and MANETs}

P2P and MANETs both exhibit a constantly changing topology. In P2P, the changes in its application layer topology are caused by end-users frequently logging on and off the system. It is also caused by dynamic IP address assignment by DHCP servers to end-users. On the other hand, changes in the MANETs network layer topology are mainly caused by node mobility.

Both P2P and MANETs depend on flooding to achieve routing functionalities. In $\mathrm{P} 2 \mathrm{P}$, the application layer topology is usually flooded to search for requested files or services, whereas in MANETs, the network layer topology is flooded to find the route to a specific end-host. 


\subsection{P2P over MANETs}

As we have seen in the previous section, P2P and MANETs are two relatively new networking technologies aimed to provide connections between end systems in a completely decentralized manner. However they differ in their "raison d'etre" and their implementation layer in the protocol stack. P2P systems were developed for the wireline networks; they create a logical infrastructure to provide services. On the other hand, MANETs create a physical infrastructure to provide connectivity in wireless environments. In order to run P2P applications on infrastructureless mobile nodes, we need to use a MANET routing protocol to provide network level connectivity. Mixing P2P with MANETs will degrade the performance of P2P systems because of the added limitations of MANETs such as frequent physical link breaks due to node mobility, limited bandwidth of the wireless links, limited battery and limited processing power of the mobile nodes.

Although that using P2P over MANETs does not usually result in the greatest performance, yet it is a very valuable tool for obtaining desired content and resources in the completely decentralized MANET environment. Compared to the wireline networks where search engines can be used on the Internet to search for content online, no such service yet exist for MANETs. Most of the times MANET users do not know the IP address of a specific host they want to communicate with, instead they might just know the kind of service or resource they are looking for. P2P applications can play an intermediary role between the mobile users and the MANET routing protocols and act as a search engine in the distributed MANET environment. 


\section{Chapter 4 File Sharing in P2P over MANETs}

Earlier P2P File-Sharing systems such as Napster and Gnutella were mainly designed for the wireline networks. They assume stable network connections and are not affected by any mismatch between the physical network topology and the logical P2P topology. The reason for this is that the length of the route or how many hops there are in between, does not usually affect the reliability of the wireline connections. As a result of the above assumption, wireline $\mathrm{P} 2 \mathrm{P}$ systems were not designed to account for node mobility. The only cause for a dynamic topology in wireline P2P systems is when nodes join or leave the overlay network. Because link quality typically do not change over the course of file downloading in wireline systems, it is automatically assumed that path failures only occur as a result of nodes leaving the overlay P2P network. At such occasions, the requesting node either resumes the downloading process from another peer or starts a new file search process to get a new path to another node that can supply the same requested file. On the other hand, in the wireless MANET environment, it is far more important to have the $\mathrm{P} 2 \mathrm{P}$ connections between any two end hosts taking part in a file-transfer activity to follow the best physical topology with as less nodes in between as possible and as good connections as possible.

Due to the above-mentioned reasons, a specific need was created for a special kind of P2P file-sharing protocol that can address the special characteristics of the MANETs. Two pioneering protocols have been developed in this area to fulfill this need; they are the ORION protocol [1] developed at the university of Dortmund in Germany and the MPP protocol [3], [9] developed at the Technical University of Munich in 
Germany. In the next two sections we are going to introduce both protocols in detail and then we will introduce our extension to the ORION protocol (ORION+) that provides better performance to the mobile nodes of the MANETs by making the best use of the available file transfer routes given by ORION.

\subsection{The MPP P2P File Sharing Protocol [3] [9]}

MPP is a P2P overlay networking system that was designed specifically for MANETs. It facilitates end-to-end communications between mobile nodes for the purpose of file sharing. MPP splits the communication protocol stack in two sections, one for handling data traffic and the other one for handling routing messages. The architecture of the 2-sided protocol stack used by MPP is illustrated in figure 4.1.

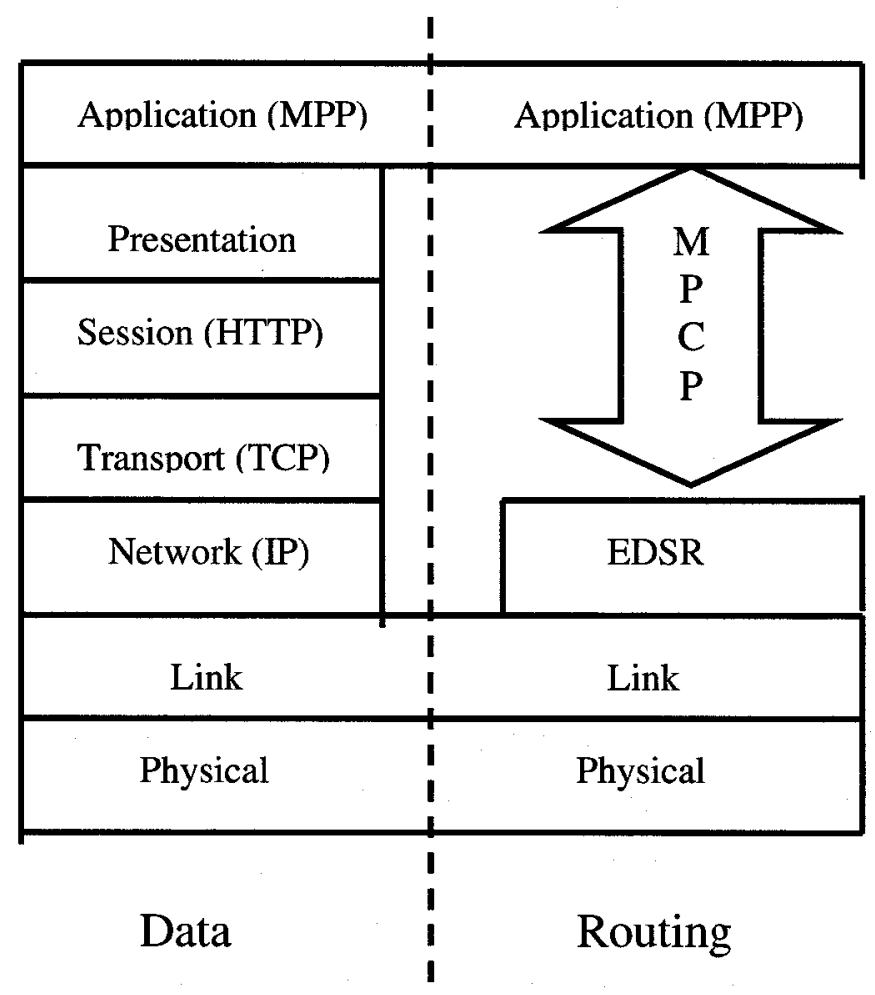

Figure 4.1 Layered structure of MPP 
The MPP protocol uses existing technologies (HTTP, TCP and IP) for the data transfer part and it introduces a cross layering mechanism for establishing the P2P overlay network and to handle the query and response messages during the file search process (the routing part). The cross layering interaction in MPP takes place between the P2P application layer (MPP) and the networking layer routing protocol (EDSR). By making the application layer aware of available network layer routes that can be used for file transfer; the protocol is able to use the overlay connection that corresponds to the best physical links available. The MPP protocol consists of thee components: The MPP application layer component, The EDSR network layer routing protocol component, and the MPCP interlayer communication protocol component. The following is a brief introduction to the three main components of the MPP protocol.

\subsubsection{The MPP application layer protocol}

The MPP application layer protocol is responsible for handling the file search messages (QUERY and REPLY) by forwarding and receiving them via the MPCP interlayer communication protocol (using the routing part of the MPP protocol stack). It is also responsible for handling the actual file transfer (using the data part of the MPP protocol stack). For file transfer, MPP uses the HTTP protocol on top of TCP because HTTP is simple to implement, well tested and the content range header of HTTP allows the application layer to resume downloading seamlessly in case of path failures. 


\subsubsection{The EDSR network layer protocol}

The EDSR (Extended DSR) network layer routing protocol is based on the DSR routing protocol for MANETs. It was developed especially to work with the MPP protocol and it allows for finding end hosts based on other criteria than their IP addresses. It introduces three new message types used for file searching. They are the Search Request (SREQ), File Reply (FREP) and Hash Request (HREQ).

The SREQ message is based on the RREQ message of the DSR protocol, it specifies additional search criteria other than just the end system IP address. Such search criteria include the requested file type (audio, video, doc) and a search string to describe the requested file. Similar to the RREQ of the DSR protocol, the SREQ message carries an up-to-date list of IP addresses of all the intermediate nodes it have visited so far. The complete source route from the node having the requested file to the requesting node can be determined by reversing the list of IP addresses carried with the SREQ message as in the DSR protocol.

The FREP message is also based on the RREP message of the DSR protocol. It can only be created by a node having the requested file specified in the SREQ message and it includes all the necessary information about the requested file (name, size, 128 bit hash value as a fingerprint of the file) and how to establish a connection with the node providing it. 
Finally, the HREQ message is a special version of the SREQ message. It includes only the size and a 128-bit hash value of the requested file as the search criterion. This message is only helpful to search for new hosts after link failures happen.

\subsubsection{The MPCP interlayer communication protocol}

The MPCP interlayer communication protocol is the communication channel between the MPP application layer protocol and the EDSR network layer routing protocol. It is responsible for registering the MPP application with the EDSR routing protocol and it also communicates all file search messages between MPP and EDSR during the file search process.

\subsubsection{Protocol Operation}

On startup, the MPP application layer protocol of mobile nodes wishing to take part in the P2P overlay network must register with their corresponding EDSR network layer routing protocol via MPCP. Upon registration, EDSR will be able to exchange file search messages with MPP via MPCP. When a user initializes a search request by sending a text string describing the requested file to EDSR via MPCP, EDSR will transform the text string into a SREQ message and flood it through the network just like the RREQ in the DSR protocol. EDSR nodes receiving the flooded SREQ message will send it to their corresponding MPP protocol if such one is registered and then they will carry on flooding it to their neighbors. Only registered MPP application layer protocols having the requested file specified in the SREQ are allowed to respond with a FREP message to the requesting node using the source route carried with the SREQ to reach it. 
Since the FREP message has all the necessary information about the requested file and how to reach the node having it, upon receiving the FREP message at the requesting node, it can communicate with the source node of the FREP and start downloading the file from it using the data transfer part of the MPP protocol stack. Figure 4.2 shows the process of searching and transferring files within the mobile P2P network as a message sequence chart.

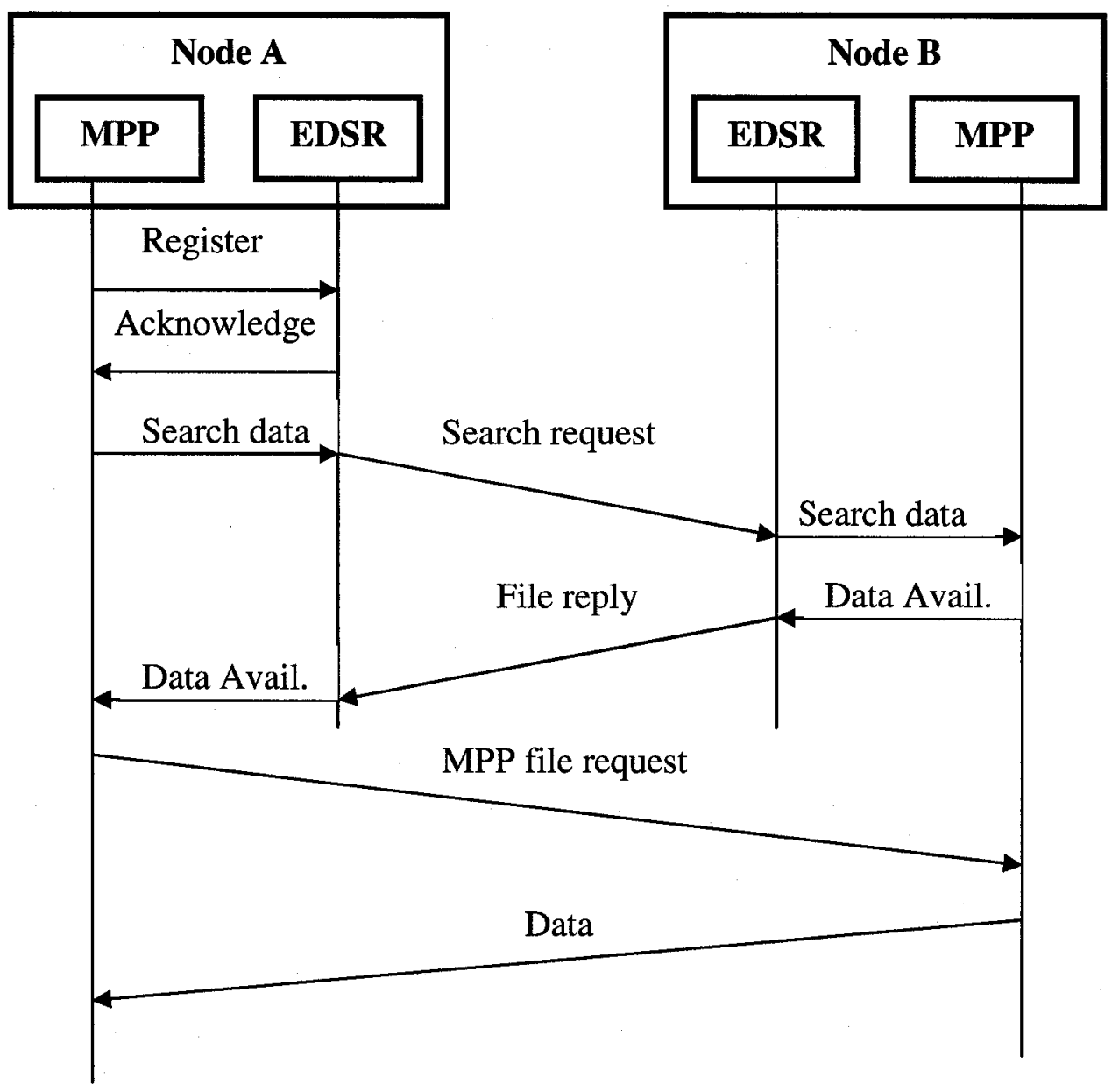

Figure 4.2 Message sequence chart for data search and download in MPP 


\subsubsection{Recovery from link failures}

MPP uses two different path recovery modes in order to resume file downloads after a link failure. They are the Static Downloading mode and the Dynamic Downloading mode. We are going to introduce them here in detail.

\subsubsection{Static Downloading}

In the static downloading mode, after issuing a file search request, the requesting node caches all the received FREP messages so that it can use their corresponding paths to resolve path failures when they happen. If the current path being used by the requesting node fails, another one will be ready to take its place to resume the file download immediately. When the requesting node runs out of cached FREPs and the requested file is not yet downloaded, a new file search must be generated (this time using a HREQ message instead of a SREQ since the hash value of the requested file is already known) by the requesting node to discover new source nodes to download the file from.

\subsubsection{Dynamic Downloading}

In the dynamic downloading mode, after issuing a file search request, the requesting node only uses the path of the first FREP it receives and discards later arriving FREPs. Every time a path failure happens, new HREQ message must be flooded throughout the network to get a fresh path. 


\subsection{The ORION P2P File Sharing Protocol}

The main advantage of the ORION (Optimized Routing Independent Overlay Network) P2P file sharing protocol over the regular file sharing protocols designed for wireline networks is that the established overlay network closely matches the underlying physical network topology. This match between the logical overlay network and the underlying physical connections is of great importance in the case of MANETs due to the known limitations of wireless communication environments. To achieve this match, the protocol combines the application layer query processing with the network layer route discovery process. The ORION protocol employs a File-Depository that is maintained by each node running the protocol, this depository contains all the files that the node is willing to share with other peers in the ORION P2P network. It also employs a ResponseRouting table and a File-Routing table at each node taking part in the file-sharing network. The Response and File routing tables are used to route control and data packets between the nodes running the ORION protocol. The protocol runs in two phases, the "File-Search" phase and the "File-Transfer" phase. Before explaining the two phases of ORION in detail, we first introduce here the different message types used by it.

\section{ORION Messages}

- QUERY: Contains a query string that have one or more keywords describing the requested file

- RESPONSE: Contains unique identifiers of one or more files matching the keywords of the query message

- DATA_REQUEST: sent by the requesting node to request transmission of a single part of the requested file 
- DATA_REPLY: Contains the requested part of the file sent back to the requesting node

- ROUTE_ERROR: Used to inform the requesting node that the path used by its current next-hop is broken. An alternative nexthop must be used or a re-query must be started if no other next-hops are remained

\subsubsection{The ORION File Search Phase [1]}

In ORION, each sharable file in a file-depository is associated with a unique file identifier. During the File-Search phase, a requesting node will flood a link-layer QUERY message throughout the network. Flooded Query messages typically include a query string that contains one or more keywords describing the requested file. Upon receiving a QUERY message at the application layer, a node should first respond with a RESPONSE message containing one or more unique identifiers of local files matching the QUERY keywords if it has such files, and then carries on flooding the network with the received QUERY message.

To eliminate multiple processing of ORION messages by the mobile nodes, each ORION message (including QUERY) must contain a SRC field and a SEQ field to uniquely identify each packet. The SRC field contains a unique identifier of the node that generated the message and the SEQ field contains a sequence number that is uniquely increasing for each node. 
To illustrate the operation of the File-Search phase we consider a network of four nodes as in Figure 4.3 where the small rectangles beside each node represent the local file depository of that node. Assuming that node " $A$ " issues a QUERY message for files $1,2,3$ and 4. The QUERY message will be distributed to all the neighbors of node "A" using link-layer flooding; in this case, only node " $B$ ". Node " $B$ " then will check it's local file depository to see if it can respond to the QUERY message and finds out that it can send only a partial RESPONSE message back to node "A" for files 1 and 2 (Figure 4.4). Node "B" will also carry on forwarding the QUERY message to its own neighbors. A Response-Routing table and a File-Routing table will also be created at node "B" to route subsequent ORION messages. The Response-Routing table will have node " $A$ " as the next hop in the reverse path for all the requested files $1-4$ while a File-Routing table will be created for files 3 and 4 upon receiving a corresponding RESPONSE message for them in reply to the forwarded QUERY message.

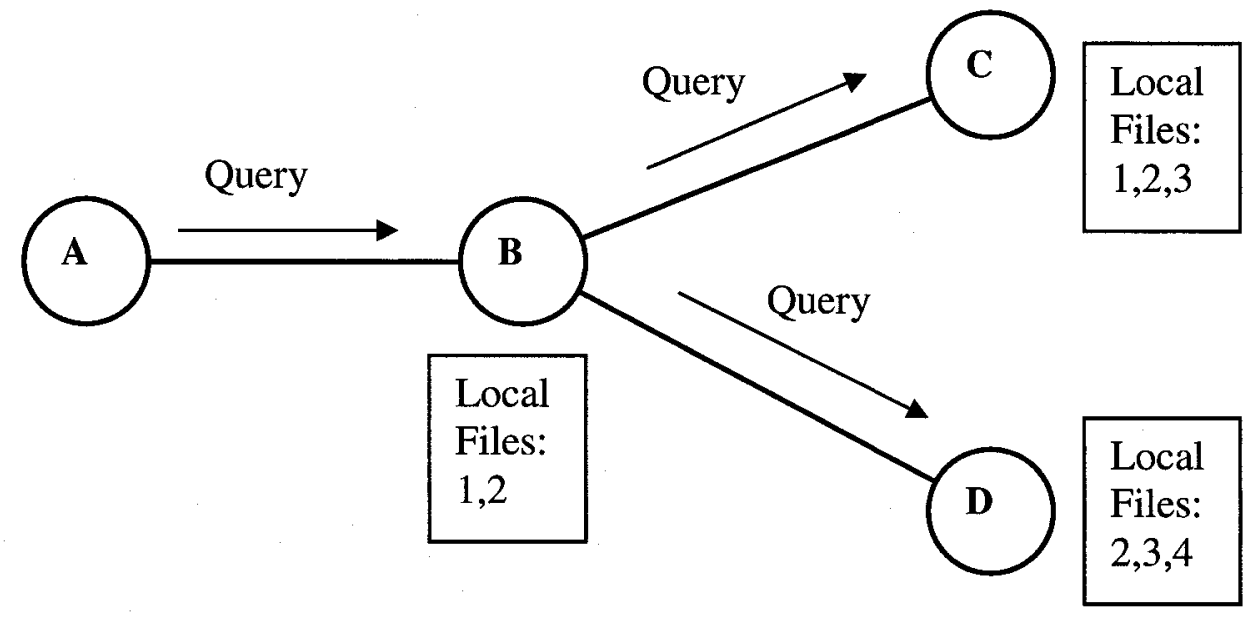

Figure 4.3 Node A floods a QUERY message for files 1, 2, 3 and 4 


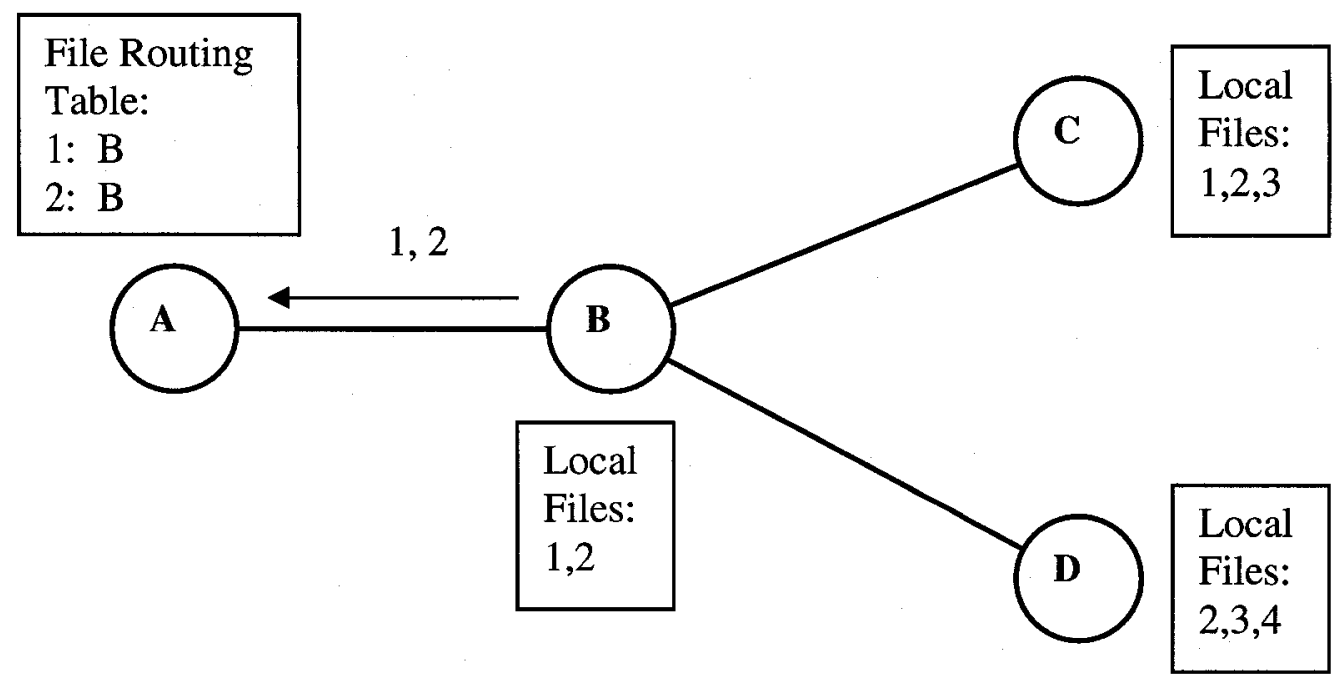

Figure 4.4 Node B sends RESPONSE message with identifiers of local files

When node " $C$ " receives the QUERY message forwarded to it from " $B$ ", it will find out that it can send a RESPONSE message back to node " $B$ " in regard to files 1,2 and 3 (Figure 4.5). It will also set up a Response-Routing table for files 1, 2 and 3 and have node " $B$ " as the next hop in the reverse path direction. When node " $B$ " receives the RESPONSE message from "C", it will inspect it and find that file 3 is still unknown to it, so it will forward a reduced RESPONSE message to node " $\mathrm{A}$ " containing only file 3 and it will also have node "C" as the next hop for file 3 in its own file-routing table. Since node " $\mathrm{C}$ " is only connected to " $\mathrm{B}$ ", it cannot propagate the QUERY message any further. 


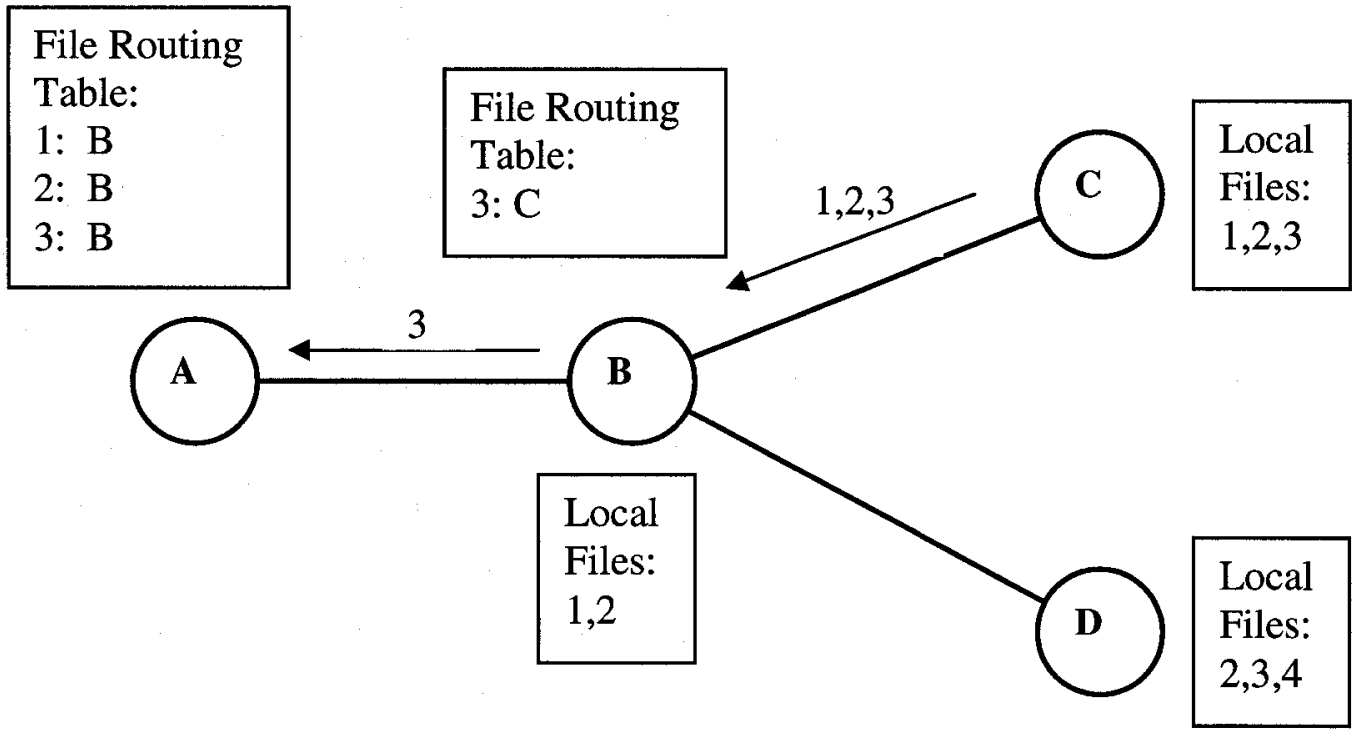

Figure 4.5 Node C sends RESPONSE message back to node $B$

Similar to node "C", node "D" will receive the QUERY message from node "B" and finds out that it can send a RESPONSE message regarding files 2, 3 and 4 back to node "B" (Figure 4.6). It will also set up a Response-Routing table for files 2, 3 and 4 and have node " $B$ " as the next hop in the reverse path direction to node " $A$ ". When node " $B$ " receives the RESPONSE message for files 2, 3 and 4 from node "D", it will inspect it and find that it is only missing file 4, so it will forward a reduced RESPONSE message to node " $A$ " containing only file " 4 ". Node "B" will also put node " $D$ " as the best next hop for file 4 and second-best next hop for file 3 (after node "C") in its own file-routing table. Similar to node " $C$ " since node " $D$ " is only connected to "B", it cannot propagate the QUERY message any further. 


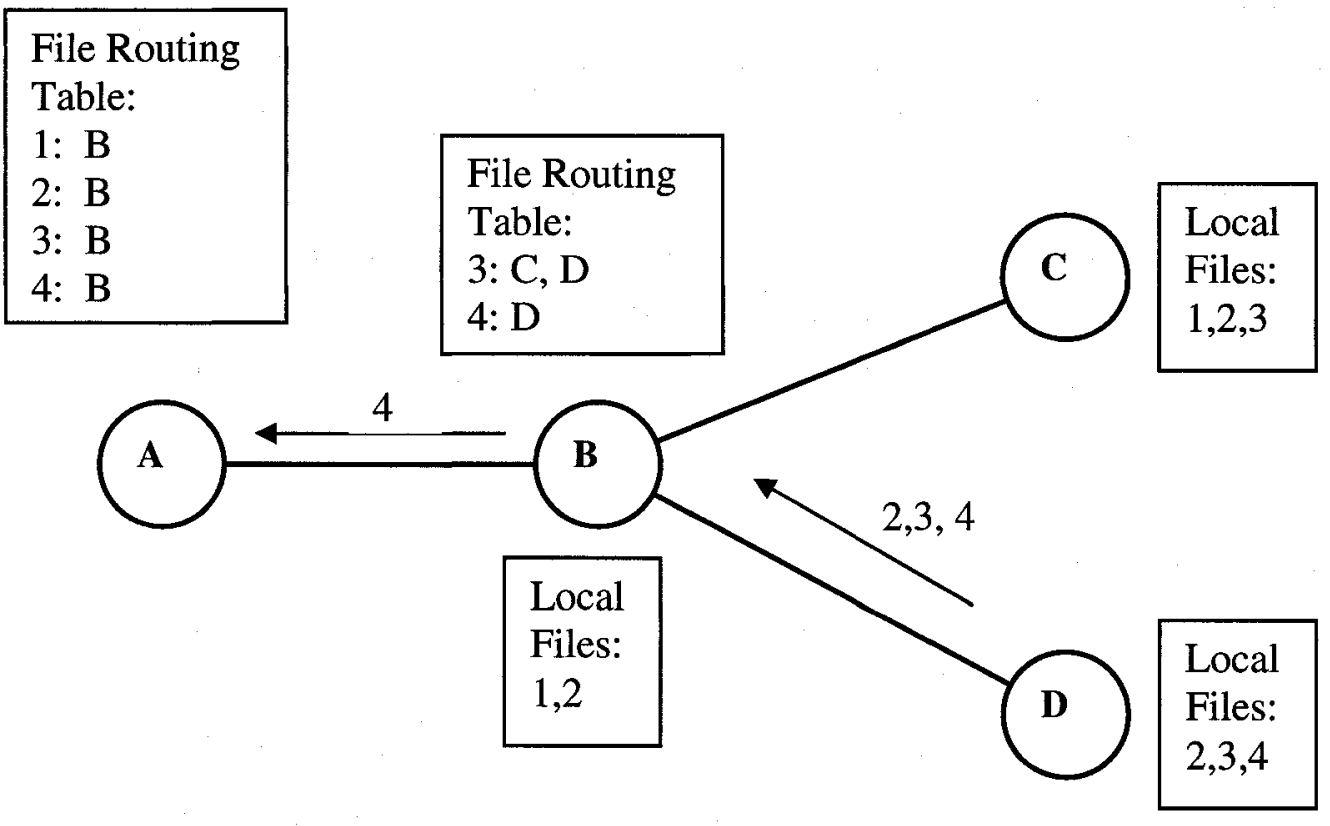

Figure 4.6 Node D sends RESPONSE message back to node B

\subsubsection{The ORION File-Transfer phase [1]}

The ORION protocol breaks down each sharable file into data chunks equal to the MTU size of the physical link connecting the mobile nodes together, so that each part of the file can be requested and received in a single DATA_REQUEST - DATA_REPLY messaging operation. The file transfer phase works as the following, for each part of a requested file, the requesting node will have to send a DATA_REQUEST message for that part along the path determined by the file routing tables starting from the requesting node until it arrives at a node that have the requested file. When a certain node that has the requested file receives the DATA_REQUEST message for a specific part of that file, it will reply with a DATA_REPLY message that contains the requested part of that file. The DATA_REPLY message will be routed back to the requesting node along the reverse path determined by the reverse routing tables from the node having the requested 
file to the node requesting the file. This operation must be repeated until all parts of the requested file are received.

To further illustrate the operation of the file-transfer phase, consider the network presented in Figure 4.3. After running the File-Search phase by node " $A$ " for files $1,2,3$ and 4, Now if node "A" wants to get file 3, it will send a DATA_REQUEST message for the first part of the file to its best next-hop for that file as determined by its file-routing table (node "B"). After receiving the DATA_REQUEST message, and since node "B" does not have the requested file, it will forward the message to its best next-hop for that file as determined by its own file-routing table (node " $\mathrm{C}$ ", since it was the first to respond to node "B" regarding file 3). When node "C" receives the DATA_REQUEST message, and since it has the requested file, it will send back a DATA_REPLY message containing the requested part of the file along the reverse path to the requesting node as determined by its response routing table (node "B"). After receiving the DATA_REPLY message at node "B", it will forward it towards the requesting node along the reverse path as determined by its response routing table (node "A"). This sequence of DATA_REQUEST and DATA_REPLY must be repeated until all parts of the requested file are received by the requesting node.

\subsubsection{Route maintenance during the File Transfer phase [1]}

In the above illustration of how the file transfer phase is handled, we assumed a problem-free operation of the protocol. In other words, we did not take into consideration what would happen if the link between a node and its best next-hop becomes unavailable 
due to node mobility or bad channel conditions. In this section we will show how the ORION protocol can handle such situations. The ORION protocol uses link-layer feedback as a way to ensure connectivity and correct reception of messages between mobile nodes (e.g.; RTS/CTS/ACK of the 802.11 MAC protocol). If a certain attempt to forward a DATA_REQUEST message fails, the corresponding entry in the file-routing table will be deleted and the next-best entry in the table will be used instead. If no more entries remain in the file-routing table for a certain file, a ROUTE_ERROR message will be sent along the reverse path direction to the requesting node. The ROUTE_ERROR message could be dropped by an intermediate node if that node can locally recover from the link failure by using an alternative route to forward the DATA_REQUEST message. If local recovery by an intermediate node is not possible, the ROUTE_ERROR message will eventually reach to the requesting node, upon receiving the ROUTE_ERROR message, the requesting node will decide either to initiate a new file-search phase to refresh its file-routing tables or to abandon the file downloading process altogether. The second run of the file search phase is called a Re-Query and it is a special case of the file search phase where the QUERY message flooded through the network contains only the unique file identifier of the requested file as the search criteria instead of a keyword to describe the file.

To illustrate more on this point lets consider again the network given by Figure 4.3, after running the previous File-Search phase for files $1,2,3$ and 4 by node " $A$ ", suppose that the wireless link between nodes " $\mathrm{B}$ " and " $\mathrm{C}$ " suffers a failure. In this case, when node "A" sends a DATA_REQUEST message for a certain part of file 3, this 
message will arrive at node "B" as in the previous problem-free scenario, but at this time, when node "B" tries to forward this DATA_REQUEST message to its best next-hop node for this file as given by its file routing table (node "C"), it will get an error from the link layer because of the broken link between the two nodes. At this point, node " $\mathrm{B}$ " will know that the link is no longer there, so it will delete node "C" as its best next-hop for file 3 and node " $D$ " will become the new best next-hop. Now node " $B$ " will try to forward the same DATA_REQUEST message to the now best next-hop for file 3 (node " $D$ "). If the link between nodes " $B$ " and " $D$ " is still up, node " $D$ " will receive the DATA_REQUEST message and respond to it with a DATA_REPLY message as in the problem-free scenario discussed before, otherwise, if the link between nodes " $B$ " and " $D$ " suffers a failure too just like the link between nodes " $B$ " and " $C$ ", then another link layer error will result after the attempt to forward the DATA_REQUEST message to node "D". If the later case happened and node " $\mathrm{B}$ " is left with no next hops in its file routing table for file 3, it will send a ROUTE_ERROR message back to the requesting node (node "A") along the reverse path. Upon receiving the ROUTE_ERROR message, the requesting node may choose to abandon the file transfer altogether or issue a new requery phase to refresh the file routing tables.

\subsubsection{Packet Scheduling and Loss recovery [1]}

ORION implements a packet-scheduling algorithm that prevents it from waiting indefinitely for lost or delayed data packets. Since that each shared file on the ORION system is split into many parts that are requested sequentially by the requesting nodes until all parts are received, to avoid the wait if one part of a requested file is lost or 
delayed, the ORION protocol sends out the DATA_REQUEST messages in a round robin fashion without having to wait for DATA_REPLYs for each part before sending a DATA_REQUEST for the following one. The time interval between any two sequential DATA_REQUESTs equals the average time between sending out a DATA_REQUEST and receiving its corresponding DATA_REPLY message. This way there is enough time allowed for each DATA_REPLY message to arrive at the requesting node before requesting it for a second time if it does not arrive.

\subsection{Current Path Selection and Recovery Mechanisms Used by MPP and ORION}

A common denominator between all P2P applications (except BitTorrent) is that they usually run in two stages, the file search (query) stage and the file download stage. The purpose of the file search stage is to acquire a set of possible paths over which a certain file can be downloaded. We refer to the way by which a requesting node deals with the set of available paths acquired by the file search stage as the "Path Selection and Recovery" mechanism. Because of the constantly changing topology of the MANETs P2P systems, path selection and recovery mechanisms are used by the requesting nodes to decide which path they are going to use first to download a certain file and how to recover from path failures when they happen. Until now, existing P2P file sharing protocols for MANETs (MPP and ORION) have followed only two techniques for path selection and recovery, they can be referred to as the "aggressive" and the "reactive" techniques. We are going to discuss these two techniques next in detail. 


\subsubsection{Aggressive Path Selection and Recovery}

In the aggressive technique (used by the MPP protocol in its Dynamic downloading mode), only the first path acquired by the file search process is used for file transfer, all subsequently arriving paths are discarded. In a case of path failure, a network-wide re-flooding must be done to get a new path. This technique follows the assumption that the first path acquired by the file search process will almost always have the best connection to the requested file. Although that this technique always uses the best path available in the network to retrieve the requested file, it usually requires frequent network-wide flooding because a re-flooding is required each time the used path fails. Existing research [18] have shown that flooding messages on today's wireless networks (using 802.11 standard MAC layer) have a halting effect on the network because nodes overhearing a transmission attempt must refrain from sending any messages until the channel is clear. This waiting process could potentially take a long time if the transmission is taking place over slow physical links making the halting effect on neighboring nodes even greater.

\subsubsection{Reactive Path Selection and Recovery}

In the reactive technique (used by ORION and MPP in its static downloading mode), paths acquired by the file search process are cached in at the requesting node and are ordered on a first-come-first-serve basis according to the arrival time of their corresponding reply messages. They are then used one by one on a sequential basis starting with the first path to be acquired. A network-wide re-flooding to get fresh paths is only required after all cached paths are broken. While the reactive technique uses less 
frequent network-wide flooding because usually there is more than one path available to be used for file transfer, the requesting node will usually have to deal with slow paths and wait for them to break before it can move one step down the list of available paths and try the next one. This sequential behavior of path selection is there because the requesting node has no way of ordering the set of available paths other than by means of the arrival time of their corresponding reply messages. The main problem with this sequential behavior of the reactive technique is that due to node mobility, the quality of the acquired paths changes over time, and by the time a certain path is to be used, it is usually broken or has a weak connection.

\subsection{ORION vs. MPP}

Since the two P2P protocols (ORION and MPP) were developed for the MANET environment, they share a lot of common attributes; the most important ones are the optimization of the used physical links and the usage of similar path selection and recovery mechanisms. However, there are also some key design differences between them. First, MPP is a cross-layer solution; it depends on the close interaction between the application layer and the network layer to help it achieve the best physical path available. On the other hand, ORION is not a cross-layer solution and it maintains standard interfaces between different layers. Second, MPP is strongly coupled with the DSR network layer routing protocol for MANETs; it requires that all mobile nodes wishing to participate in the overlay $\mathrm{P} 2 \mathrm{P}$ network to be running the DSR protocol. On the other hand, ORION does not require a specific network layer routing protocol to work with because it was strictly implemented as an application layer solution. In this thesis, we 
introduce a new addition to the reactive path selection and recovery mechanism that is currently being used by both of MPP and ORION. Because of the limited nature of MPP since it only works with the DSR network layer protocol, we have decided to work with the reactive path selection and recovery mechanism in the ORION platform to provide a more general solution that can work with any kind of MANET network layer protocol. In the next chapter, we will introduce a new extension of the ORION protocol (ORION+) that aims at improving the currently used (reactive) path selection and recovery mechanism by means of added application level unicast messaging. Since the static downloading mode of MPP uses a similar path recovery mechanism as ORION (reactive), it can also benefit from the proposed mechanism. 


\section{Chapter 5 The ORION+ Extension}

P2P File sharing applications are usually used to download large files, they usually run as background processes and they do not need any user input once they are started, so users just need to initiate the downloading process and wait for the P2P application to finish its work. Due to the fact that MANET nodes are always on the go, it is always the case that the quality of the paths gained by the file search phase will fluctuate over time until they eventually break down as a result of node mobility. Because $\mathrm{P} 2 \mathrm{P}$ applications are meant to be independent most of the time, it is important for such applications to make intelligent decisions about which paths to use for their file transfer especially in the limited resources environment of MANETs. Until now, only two extreme path selection and recovery mechanisms (the aggressive and the reactive techniques presented in chapter 4) have been used by the MANETs P2P file sharing applications. While the aggressive technique is always able to select the best path available in the network, it requires frequent network-wide flooding each time a path failure happens. The reactive technique, on the other hand does not require frequent network-wide flooding but at the same time it does not make any guarantees about the quality of its chosen paths. Figure 5.1 illustrates the tradeoff between path quality and control overhead in the aggressive and the reactive path selection and recovery mechanisms

As we have discussed in section 4.3.2, the problem with the reactive recovery mechanism is that because of the random node mobility in MANETs, the list of paths available to the requesting node at the beginning of the file downloading stage will not be 
kept in order over the course of the file transfer. In order to achieve better path selection decisions while still avoiding the frequent network-wide flooding associated with the aggressive technique, we have added a new functionality to the reactive path selection and recovery mechanism to rearrange on a periodic basis the set of available paths according to their increasing response time. Rearranging the set of available paths allows for intelligent switching between file transfer paths so that the one with the least response time is always used. As a result, no unnecessary waiting for a weak path to break is required if a better one is available. Since only those paths already known (acquired by the file search process) to the requesting node are being reordered periodically, unicast probing messages are used, as it will be explained in sections 5.2 and 5.3. We categorize our new path selection and recovery technique as "reactive with unicast probing messages" and it is the main added functionality in our new extension of the ORION protocol (ORION+). Figure 5.1 illustrates the relative position of our new path selection and recovery mechanism used in ORION+ (Reactive with unicast messaging) with respect to the old ones (aggressive and reactive).

\begin{tabular}{|c|}
\hline Reactive \\
\hline - MPP (Static \\
downloading) \\
- ORION \\
\hline
\end{tabular}

(1)

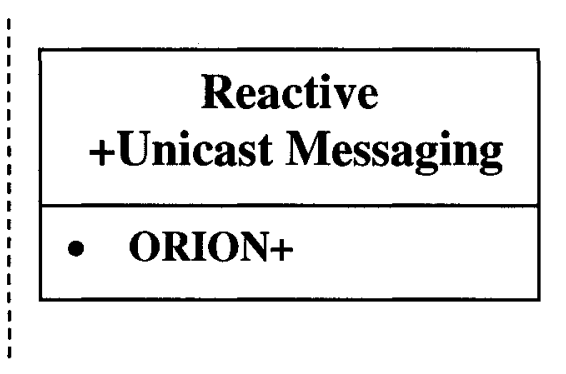

( 2 )

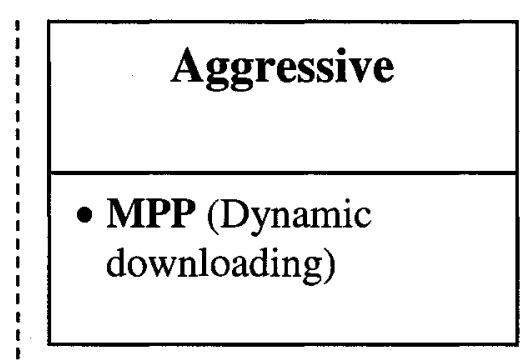

( 3 )

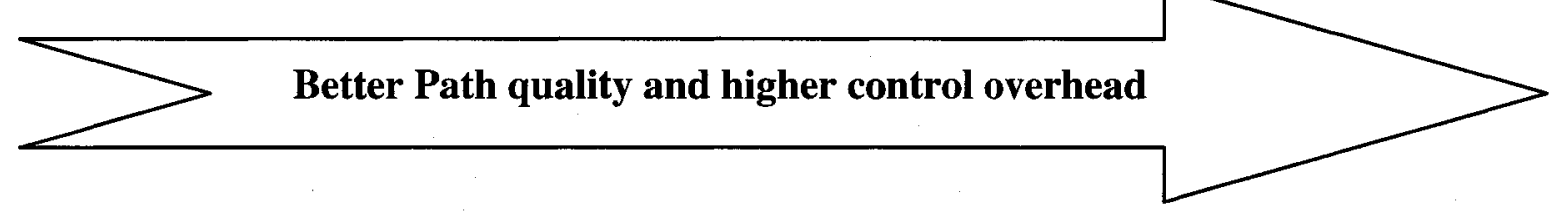

Figure 5.1 Tradeoff between different Path selection and recovery mechanisms 


\subsection{Possible Improvements for the ORION protocol}

In our effort to improve the ORION protocol, some ideas were researched but later on excluded due to different reasons; here we are going to discuss those ideas and outline the reasons why they were excluded.

\subsubsection{BitTorrent alike downloading}

BitTorrent is a popular file-sharing protocol for today's wireline Internet users. It requires the existence of a tracker server at a fixed address, which cannot be achieved in MANETS due to their dynamic nature. BitTorrent also takes advantage of the asymmetrical allocation of the available bandwidth for each Internet subscriber. Usually the portion of the available bandwidth assigned to carry downloading traffic is much greater than the one assigned for uploading traffic. BitTorrent exploits this feature to achieve multisource downloading by allowing nodes to fill up their downloading bandwidth from many sources. Since the downloading bandwidth is typically larger than the uploading one, many nodes will be required to take part in uploading to a certain node to fill up its downloading bandwidth. This technique is possible in wireline networks because of two reasons. First, In wireline networks, there exists an asymmetric bandwidth allocation where end users usually have dedicated bandwidth as determined by their service level agreement; this bandwidth is considered "wasted" if not used within a certain timeframe. On the other hand, not only that there is no distinction between uploading and downloading bandwidth in wireless networks (using 802.11 MAC layer), but the available bandwidth is also shareable by more than one node. Second, The existence of dedicated bandwidth in wireline networks allows for the requesting node to 
set up multiple circuit switched connections with other source nodes to download the requested file simultaneously from them. On the other hand, with the RTS/CTS/ACK reservation style used in today's wireless networks, only one connection is allowed to be established at a time (if no time multiplexing is used). In order to achieve multisource downloading as in wireline networks, multiradio antennas will have to be used to avoid interference, which they are not currently adopted in large by today's wireless networks.

\subsubsection{Periodic rebroadcasting to discover new sources}

While periodic rebroadcasts to discover new sources will always result in finding the best available source to download a certain file from, this technique is excluded to avoid the halting effect that periodic flooding will introduce to the network especially over slow links as discussed in section 4.3.1.

\subsection{The ORION+ Approach}

As we have explained earlier in the previous chapter, the file routing tables constructed by the file search phase usually contain many redundant routes to different mobile nodes having the same requested file. The routes in those tables are ordered according to the arrival time of their corresponding RESPONSE messages. The node from which the first RESPONSE message was received in reply to a specific file QUERY message will be stored as the best next-hop in the file routing table of that file. As following RESPONSE messages arrive, the nodes from which they came will be stored at the file-routing table as the next-best next hops and they will be ordered according to the 
arrival time of their corresponding RESPONSE messages on a first-come first-serve basis.

The problem with the above approach is that the ORION protocol is unaware of any dynamic change that may happen in the path quality of all the next-best routes, so it does not switch to a better path if one is available and it does not delete stalled routes until they are tried first after a link failure.

Our solution to this problem is based on periodically propagating a unicast LINK_QUALITY_REQ message from the requesting node of a certain file to all its nexthops for that file as given by its file-routing table. Upon receiving a LINK_QUALITY_REQ message, if a node does not have the file that is the subject of the message, it will have to forward it to all of its next hop nodes for that file as given by its file-routing table. On the other hand, if the node receiving the LINK_QUALITY_REQ message does have the file that is the subject of the message, it must send back a unicast LINK_QUALITY_REPLY message along the reverse path to the requesting node that initiated the LINK_QUALITY_REQ message. Intermediate nodes along all the paths between the requesting node and all possible suppliers of the requested file will evaluate the received LINK_QUALITY_REPLY messages and order the next-hop entries of their file-routing tables accordingly. If no LINK_QUALITY_REPLY message is received for any of the next-hop entries or if a link layer error occurred when trying to send the unicast LINK_QUALITY_REQ, the corresponding next-hop will be deleted from the file-routing table. 
Based on an average human walking speed of approximately $1 \mathrm{~m} / \mathrm{s}$, the frequency of the LINK_QUALITY_REQ messages is chosen to be every five seconds to allow for a possible change in node connectivity.

To illustrate our approach in handling the next-hop entries of the ORION file routing tables, consider the network represented in Figure 5.2

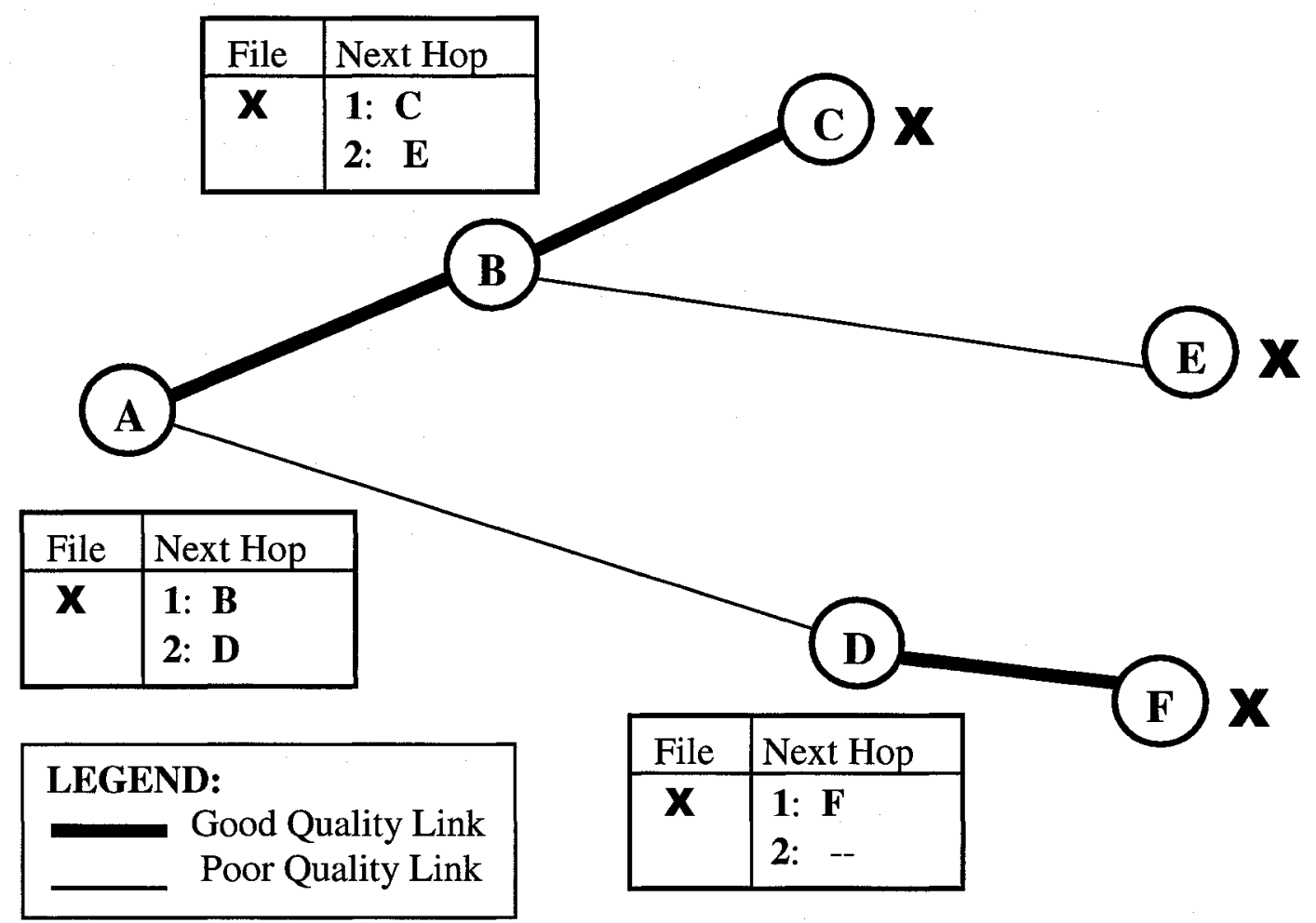

Figure 5.2 File routing tables after node $A$ initiated a file search for file " $X$ "

Here we have a six nodes network where only three nodes ("C", "E" and "F") have a certain file " $\mathrm{X}$ ". To show the different link qualities in this example, we have used thick lines to represent good quality links and thin lines to represent poor quality links. 
Suppose that node " $A$ " wants to get the file " $\mathrm{X}$ " and that it has started a file search phase looking for nodes having this file. The resulting file-routing tables constructed by the filesearch phase are as shown in figure 5.2. Here we can see that because the bandwidth of the connection between nodes " $\mathrm{B}$ " and " $\mathrm{C}$ " is better than the bandwidth of the connection between nodes " $\mathrm{B}$ " and " $\mathrm{E}$ ", node " $\mathrm{C}$ " is quicker to reply with a RESPONSE message to the QUERY forwarded to it by node "B". Hence, node "C" will be stored as the best next-hop for file " $\mathrm{X}$ " in the file-routing table of node " $\mathrm{B}$ ". The second-best next-hop will be node " $E$ " since it is slower to send its RESPONSE message to node " $B$ " because of the poor link quality between it and node "B". Similarly, because of the poor link quality between nodes "A" and "D", node "B" will send it's RESPONSE message to node " $A$ " quicker than the RESPONSE message arriving from node " $D$ ", hence node "B" will be stored as the best next-hop in the File Routing table for file " $\mathrm{X}$ " at node " $\mathrm{A}$ ".

Now suppose that the link quality between nodes " $\mathrm{B}$ " and " $\mathrm{C}$ " becomes poor because of node movement or a drop in the battery power for example while the link quality between nodes "B" and "E" gets better because of similar reasons (Figure 5.3). 


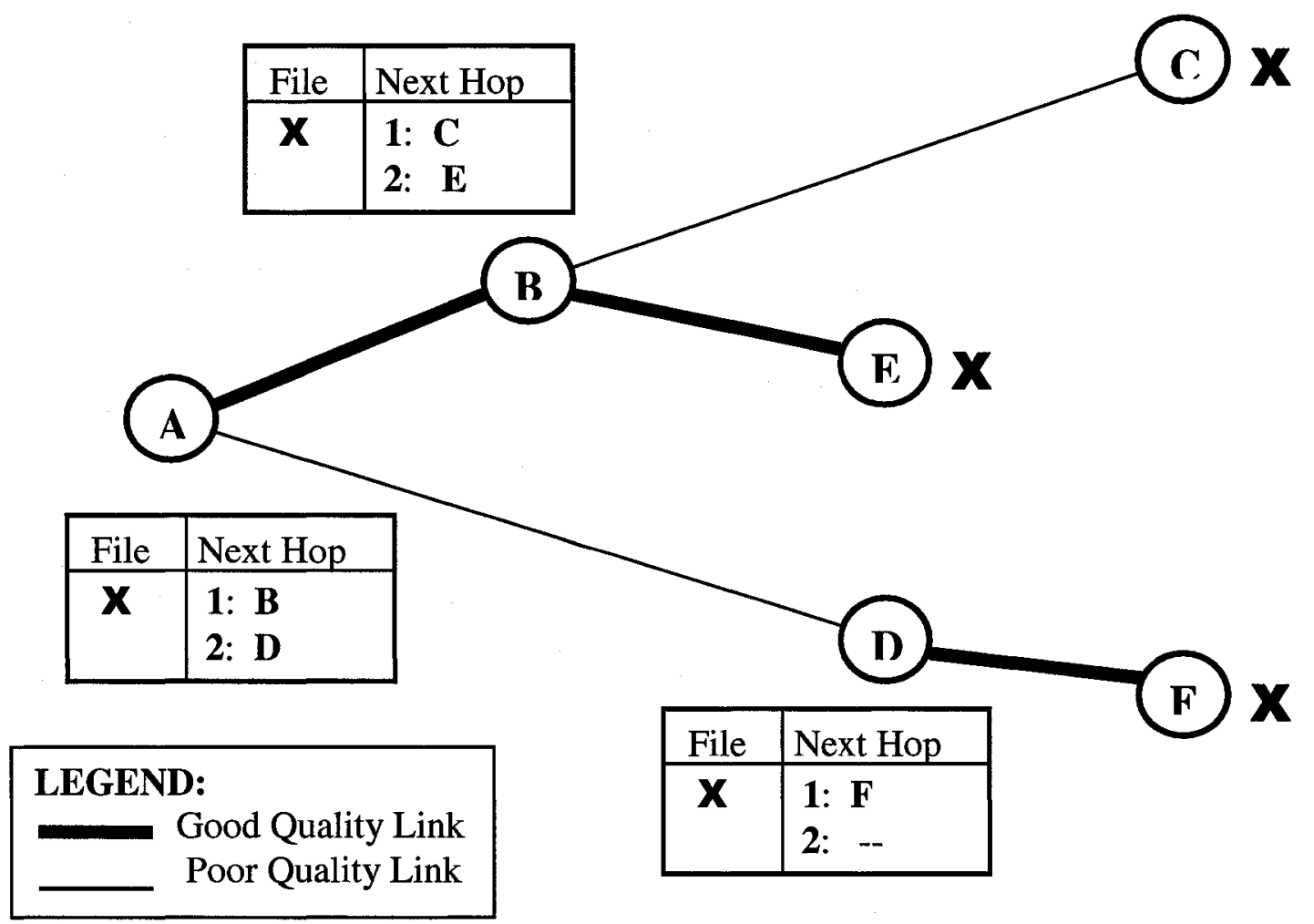

Figure 5.3 Network after change in link quality

Under the current ORION protocol, this change will not be noticed and the DATA_REQUEST messages sent by node "A" for file "X" will continue to be forwarded to node "C" instead of node " $\mathrm{E}$ " which is now offering a better connection (Figure 5.4). Unless the link between nodes " $B$ " and " $C$ " fails completely, the system will not try out the other routes that it already have for the requested file. 


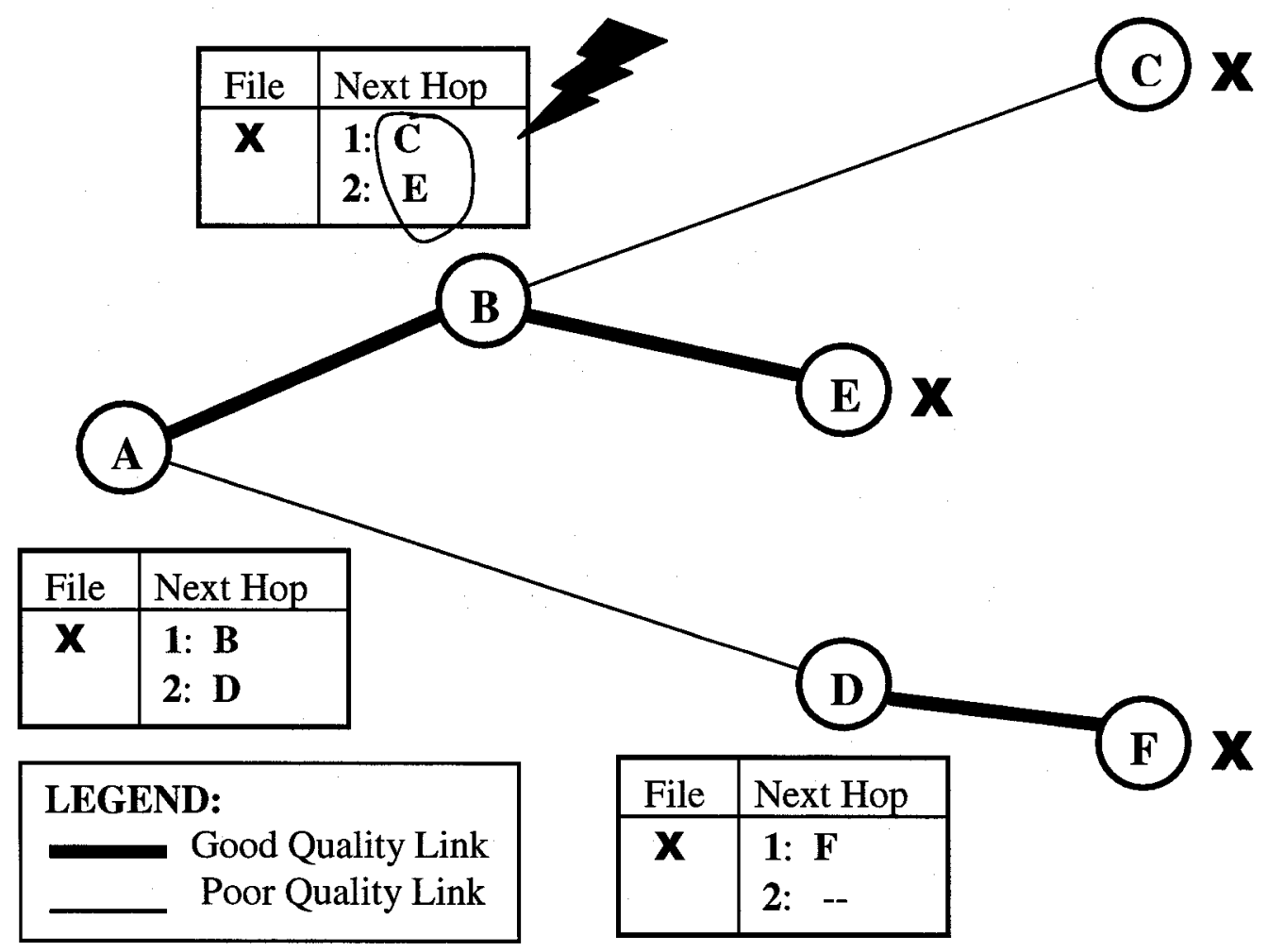

Figure 5.4 File routing tables are not changed after change in the link quality

\subsection{The ORION+ Implementation details}

In our approach to handle the file routing tables of the ORION protocol, we define two new message types for ORION, the "LINK_QUALITY_REQ" and the “LINK_QUALITY_REPLY”. The LINK_QUALITY_REQ message is generated periodically for each file being currently downloaded, it can only be generated by the requesting node (the node that originally initiated the file-search phase) and it must carry the node identification of that node as well as the identification of the requested file that it seeks to update its entries in the file-routing table. The LINK_QUALITY_REPLY message is sent in response to LINK_QUALITY_REQ and it can only be generated by nodes that have the file specified in the LINK_QUALITY_REQ message. The file- 
routing tables must also be modified to include two new time values (TS1 (Time Stamp 1) and TS2 (Time Stamp 2)) for each next-hop entry. The first time-value (TS1) is set to the current time when a node generating or forwarding a LINK_QUALITY_REQ message sends out that message to a specific next-hop. The second time-value (TS2) is set to the current time when a node receives a LINK_QUALITY_REPLY from one of the next-hops that it has previously sent a LINK_QUALITY_REQ to. The response time for each next-hop (TS2-TS1) represents the link quality and is used as the basis for ordering the available next-hops so the best one can always be used. Failure to forward LINK_QUALITY_REQ to a certain next-hop or failure to receive LINK_QUALITY_REPLY from a certain next-hop is treated exactly like the failure to forward FILE_REQ or receive FILE_REPLY messages in ORION. This helps to delete staled routes from the file-routing tables before they have to be tried after a link failure.

The purpose of the LINK_QUALITY_REQ and the LINK_QUALITY_REP probing messages is to measure the response time or the roundtrip time between sending out a LINK_QUALITY_REQ and receiving the corresponding LINK_QUALITY_REP for each available path. This roundtrip time is represented by the value (TS2 - TS1) and it is the basis for choosing which path is to be used first to download the requested file. While other metrics are also available to measure the quality of the available paths like hop-count and signal-strength. We have decided not to use them for the following reasons: 


\section{For the hop-count metric}

The hop-count metric can be misleading at times if an intermediate hop is weekly connected or badly congested. At such cases it would not be a good representative of actual path quality.

\section{For the signal-strength metric}

While the signal-strength metric will always represent correctly the actual path quality, we have decided not to use it to preserve the application level nature of the ORION protocol since getting the signal-strength information will require cross layering with the physical layer to get the required information. Also since the signal strength metric is measured on a hop-by-hop basis, it would require state maintenance as well as modifications at intermediate nodes that are not necessarily running the ORION protocol.

We have chosen the roundtrip time metric as a representative of path quality because it preserves the application layer nature of ORION, a good representative of actual link quality and it does not need any state maintenance or modifications at intermediate nodes since it is evaluated on an end-to-end basis. While many averaging techniques (that takes into account previous measurements) could be used to calculate an average for the roundtrip time, we have decided to use only the most recent measurement obtained as a representative of the current path quality. The reason for this is that because of the constantly changing physical topology in MANETs, earlier measurements of roundtrip time could be misleading. 
Figure 5.5 shows how the ORION+ technique can be used to correctly re-organize the file-routing tables after link qualities of different next-hops change. Node " $\mathrm{A}$ " as the requesting node of file " $\mathrm{X}$ " must periodically generate a LINK_QUALITY_REQ and forward it to each next-hop for that file as given by its file-routing table. The TS1 field for each next-hop must be set to the current time as the LINK_QUALITY_REQ is being sent out.

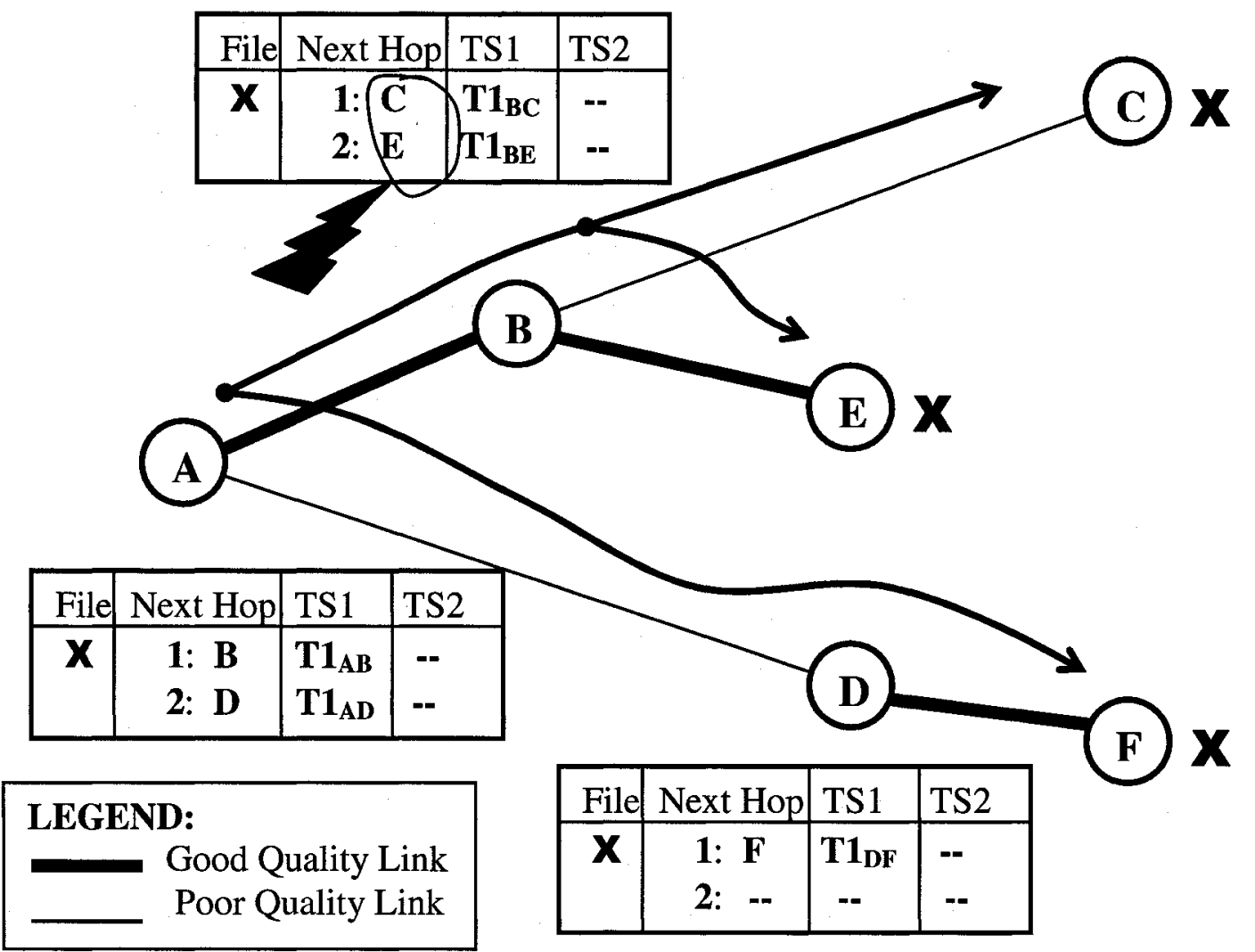

Figure 5.5 Propagation of the LINK_QUALITY_REQ message to all available next-hops for file " $x$ " 
After receiving the LINK_QUALITY_REQ, nodes "C", "E" and "F" must reply with a LINK_QUALITY_REPLY message to each node from which they have received a LINK_QUALITY_REQ. Upon receiving LINK_QUALITY_REPLY at intermediate nodes, these nodes must set the TS2 field of the next-hop from which the LINK_QUALITY_REPLY came from to the current time and forward the message along the reverse path to the requesting node. Having TS2 and TS1 now known, the response time is computed by each node and the file-routing tables are re-arranged accordingly if needed as in Figure 5.6

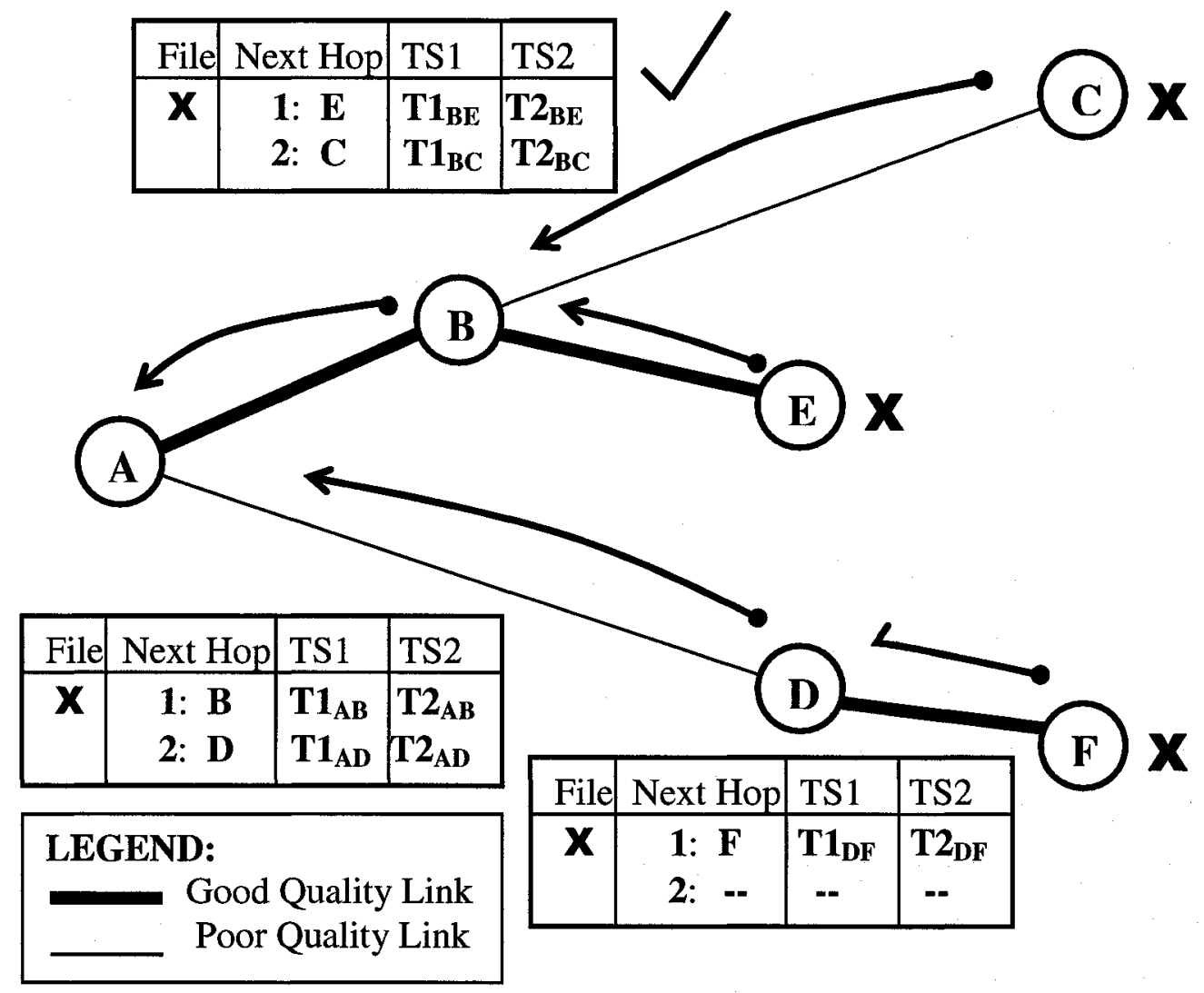

Figure 5.6 LINK_QUALITY_REPLY is sent in reply to LINK_QUALITY_REQ 


\subsection{Advantages and Disadvantages of the ORION+ approach}

\subsubsection{Advantages}

- Re-ordering of the available routes that already exist in the file routing tables so the best ones can always be used

- Extra control messages (LINK_QUALITY_REQ and LINK_QUALITY_REPLY) are unicasted to the next hop neighbors as given by the file-routing tables, So no expensive flooding is needed to learn about better routes that already exist in the network

- Stalled routes are discovered in the process, this saves time by avoiding having to try those routes if a link failure in the primary route happens.

\subsubsection{Disadvantages}

The ORION+ approach could only be useful if two conditions are satisfied. The first condition is the availability of more than one path that could be used to download the requested file. The second condition is the occurrence of a change in the quality of the available paths. If any of those two conditions are not satisfied, the operation of ORION+ will be no different than that of ORION except with the extra unicast messaging overhead. To illustrate more on the two special cases that could happen if any of the above conditions are not met, we are going to discuss them here in more detail. 


\subsubsection{The existence of only one source node in the network}

Because each node is allowed to reply only once to any new QUERY message it receives if it had the requested file, the requesting node will eventually have a number of available paths that equals the number of the source nodes in the network. Accordingly, in the special case when only one source node exists in the network, the requesting node will have only one path available for it to get its requested file. Under this special case, the probing messages of ORION+ will not be able to provide any enhanced performance since there are no alternative paths to choose from. To illustrate more on this special case, consider figure 5.7 that shows a six nodes network with the file routing tables that resulted after node " $\mathrm{A}$ " have issued a file-search for file " $\mathrm{X}$ " which happens to exist only at node "C". Here we can see that node "A" have only one available path $(A-B-C)$ over which it can get this file.

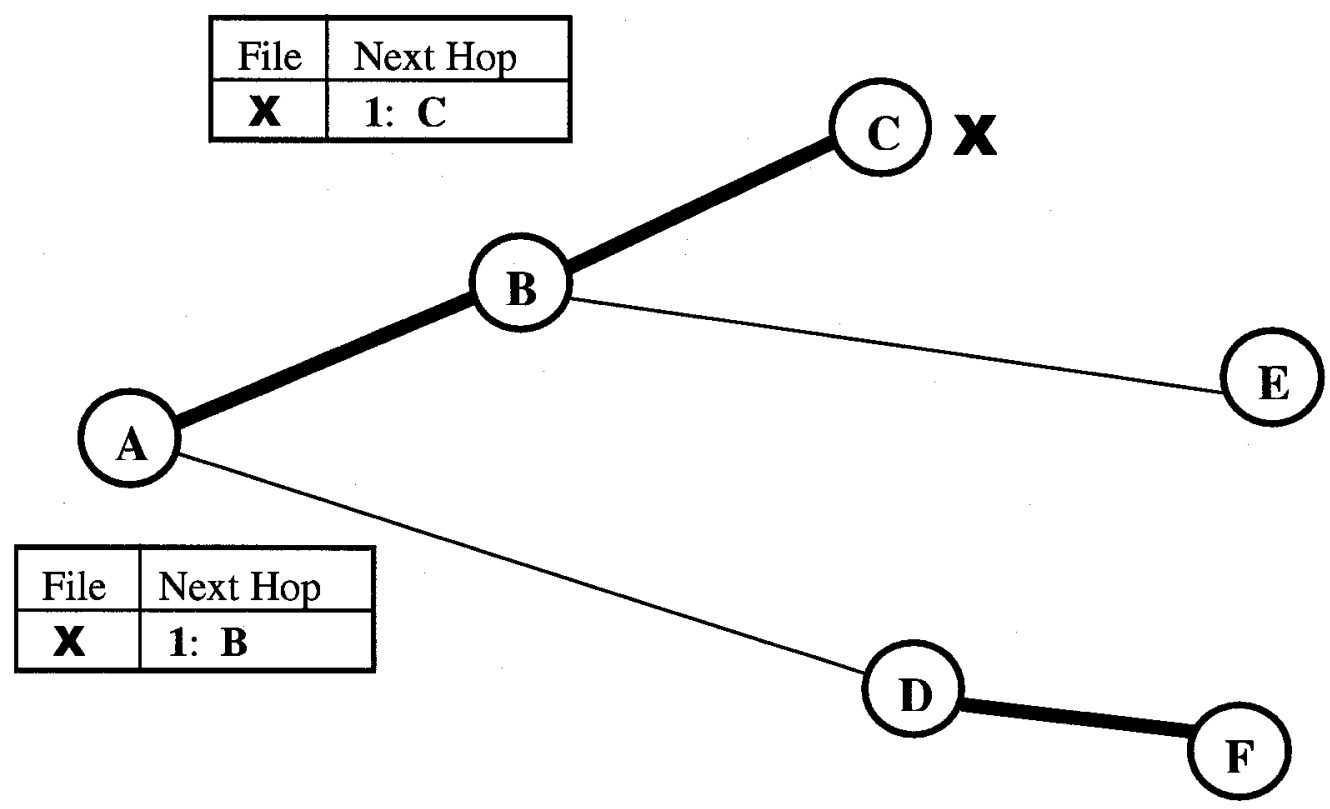

Figure 5.7 File routing tables after node $A$ initiated a file search for file " $X$ " 
Now assume that the link quality between nodes " $B$ " and " $C$ " becomes poor because of node mobility or lower battery power as in figure 5.8. Because there is only one path existing in the network for the requested file " $\mathrm{X}$ ", the ORION+ probing messages will be of no use since there are no alternative paths available. However, although that the ORION+ approach is unable to improve the file downloading performance in this special case, it does not produce considerable overhead since it only uses unicast probing messages.

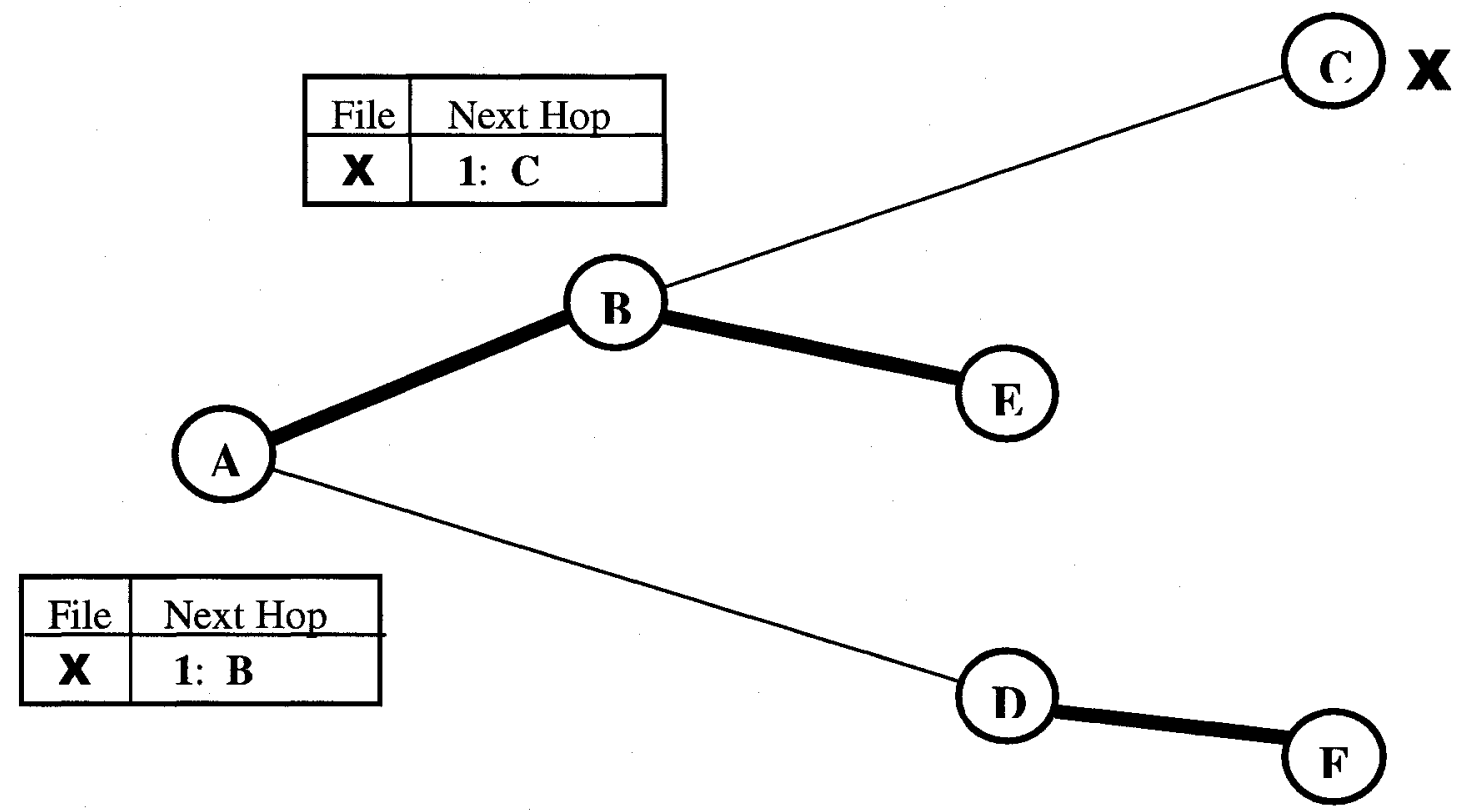

Figure 5.8 No possible improvement if the requested file is only available once

\subsubsection{No change in the quality of the available paths}

If no changes happen to the quality of the available paths (due to stable battery power levels or under no mobility conditions), the probing messages of ORION+ will continue to provide the same response time for each path and no reordering will happen. 


\section{Chapter 6 Simulation and Results}

To measure the performance of our ORION+ protocol extension against the original ORION protocol, we have used the NS-2 simulator to do some simulation experiments similar to those that were done on ORION. NS-2 is a discrete event driven network simulator initially developed by the University of California at Berkley. We have performed all our simulations using the ns- 2 version 2.29 with the ad hoc network extension from Carnegie Melon University monarch project. We have kept most of our simulation parameters in-line with those used to measure the performance of the Original ORION protocol so we can compare our results against those published for ORION.

\subsection{Simulation Model}

The ORION+ implementation resides in the application layer of ns-2. Since it has been proved by [2] that both of the main reactive routing protocols (AODV and DSR) performed similarly with ORION, we have decided to use AODV as the network layer protocol of choice for our simulation. In order to get meaningful results from our simulation, we needed to make the link quality between wireless nodes to vary depending on the physical distance between them. Unfortunately the standard ns-2 simulator does not provide this functionality, in the standard ns- 2 implementation, each node have a transmission range and nodes can only communicate to each other if they are within the transmission range of each other. Moreover, the link quality between mobile nodes does not depend on the distance between them, the link either exist if the nodes are within the transmission range of each other or does not exist if they are not within each other's transmission range. To overcome this problem, we have used the OAR multi-rate MAC 
layer extension provided by the RICE Networks Group (RNG) of RICE University. Combining the Multi-Rate MAC with the transmission range provided by ns-2, now the quality of the link between any two mobile nodes will depend on the physical distance between them until it goes away completely when the nodes go out of the transmission range of each other.

The used simulation parameters are as follows

\begin{tabular}{|l|l|}
\hline \multicolumn{1}{|c|}{ Parameter } & \multicolumn{1}{|c|}{ Set Value } \\
\hline Wireless node transmission range & $115 \mathrm{~m}$ \\
\hline Number of nodes & 50 and 100 \\
\hline Number of files & 10 \\
\hline Frequency of LINK_QUALITY_REQ & $5 \mathrm{~s}$ \\
\hline File sizes & $2 \mathrm{~KB}, 50 \mathrm{~KB}, 500 \mathrm{~KB}, 1 \mathrm{MB}, 3 \mathrm{MB}$, \\
\hline Simulation area & $10 \mathrm{MB}, 50 \mathrm{MB}$ and $100 \mathrm{MB}$ \\
\hline Mobility model & $1000 \mathrm{~m} \mathrm{x} 1000 \mathrm{~m}$ \\
\hline Maximum speed & Random waypoint \\
\hline Rest (Pause) time & $4 \mathrm{~m} / \mathrm{s}$ \\
\hline MAC protocol & $5 \mathrm{~s}$ \\
\hline Physical layer radio propagation & IEEE 802.11 (with OAR Multi-Rate \\
\hline Transmission power & extension) \\
\hline
\end{tabular}

Table 1 Simulation Parameters 


\subsection{Simulation Setup}

In our simulation we used two network sizes (50 and 100 nodes) occupying the same simulation area $\left(1000 \mathrm{~m}^{2}\right)$ to represent high and low node densities. The mobility of the nodes is according to the random waypoint mobility model. In this mobility model, the speed of the mobile nodes is chosen uniformly at random from $\left[0, S_{\max }\right]$, when a device reaches a randomly chosen destination, it pauses for a fixed amount of time $\mathrm{T}_{\text {hold }}$ before it continues to move to the next randomly selected destination with a new randomly selected speed. All simulation parameters are set according to Table (1). Each scenario was repeated 100 times with randomly generated node movement scenarios. We used 10 sharable files for both of the 50 and 100 node scenarios. We used file sizes from $2 \mathrm{~KB}$ up to $100 \mathrm{MB}$ to represent a wide range of file sizes that could be shared on the system. Each sharable file is replicated two times at the beginning of each simulation run and nodes are required to download all the 10 files from each other. We have calculated 95\% confidence intervals and indicated them on each performance curve. To simulate the division of files into pieces by the ORION protocol, we have used arrays to represent sharable files, so they can be requested one element at a time until the whole array (file) is transferred. In the following section we present our simulation results regarding to the following three metrics:

1. File transfer time

2. Percentage of successful files transferred

3. Transmitted data volume 


\subsubsection{Transfer Time}

Transfer time refers to the duration of time it takes from the beginning of the file transfer phase until the whole file is successfully downloaded. Figures 6.1 to 6.8 show the simulation results for the 50 nodes scenario and Figures 6.9 to 6.16 show the simulation results for the 100 nodes scenario for different file sizes. As we increase the file size, we can see that the original ORION protocol incurs larger transfer times than ORION+ due to the fact that it is reactive in nature. For low mobility, the transfer times goes up for both of ORION and ORION+ because when all remaining available paths are weakly connected, they take longer time to completely break and during that time they provide slower connection. Despite that, ORION+ is able to achieve lower transfer times compared to ORION because of its better path selection mechanism while better quality paths exist in the network. When increasing the speed of the mobile nodes and forcing them to run out of next hops in their file routing tables and hence generating file search phases more often, the entries at the file routing tables become more relevant causing the transfer time to go down but of course global searches must be done more often. The higher time-delay to transfer the same file sizes in high node density (100 nodes scenario) suggests that longer routes are being used to transfer the files.

In Figures 6.1 and 6.2, we see that the delay performance of both of ORION and ORION+ is the same. Because of the very small file sizes ( $2 \mathrm{~KB}$ and $50 \mathrm{~KB}$ ), the whole file is always downloaded before it is time to send out the ORION+ probing messages. In Figure 6.3 (500 KB file size), for low mobility, the whole file is successfully downloaded before there is a need to reflood the network (usage of weekly connected paths result in 
higher transfer delay). For high mobility, a reflooding is needed to get new paths (usage of fresh paths decreases the overall transfer delay). In Figure 6.4, we see that the whole file can be successfully downloaded with no need for reflooding only under very low mobility (max speed less than $1 \mathrm{~m} / \mathrm{sec}$ ), for higher max speeds, a reflooding is always required to get new paths. For file sizes $3 \mathrm{MB}$ and larger (Figures 6.5-6.8), a reflooding is always required since all paths are broken before the whole file is successfully downloaded.

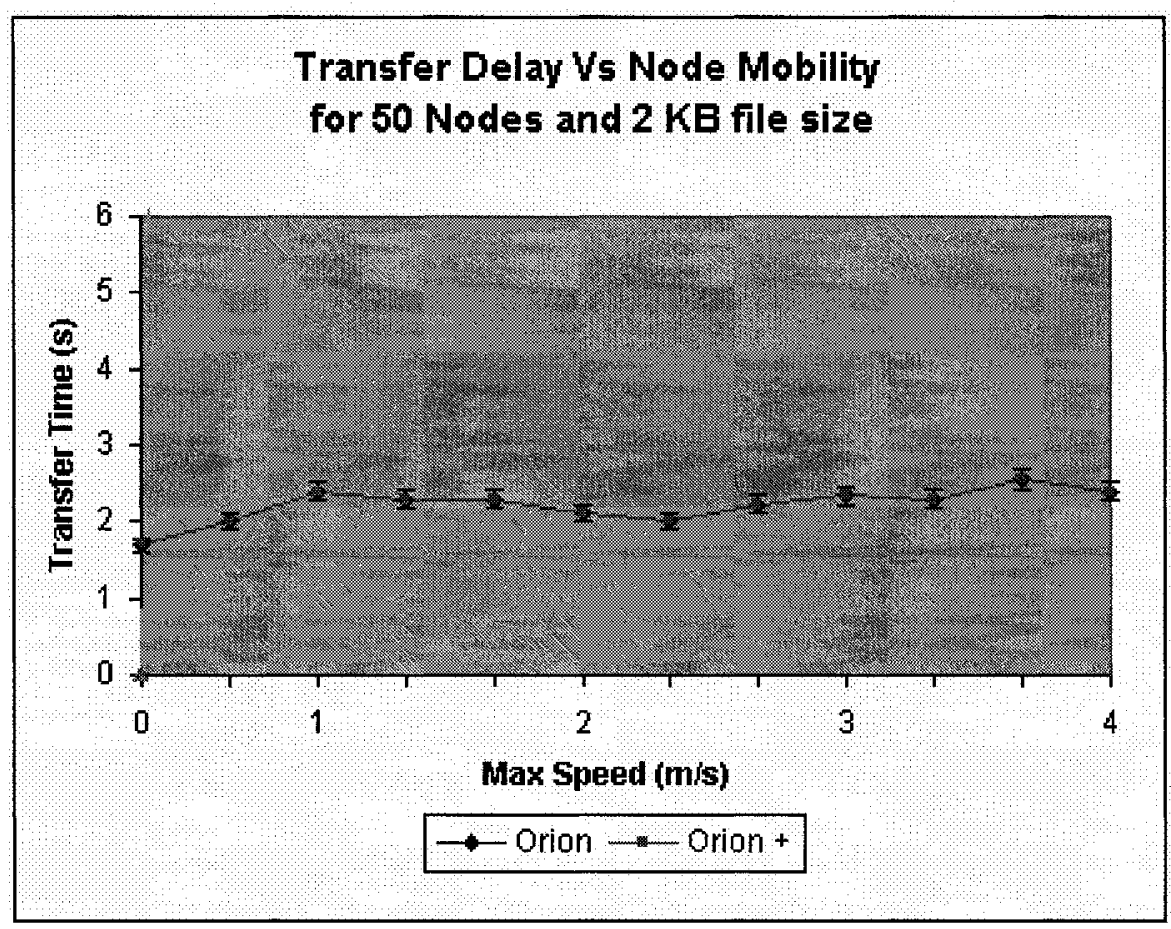

Figure 6.1 Transfer Delay vs. Node Mobility for 50 nodes and $2 \mathrm{~KB}$ file size (Results are displayed with their $95 \% \mathrm{CI}$ ) 


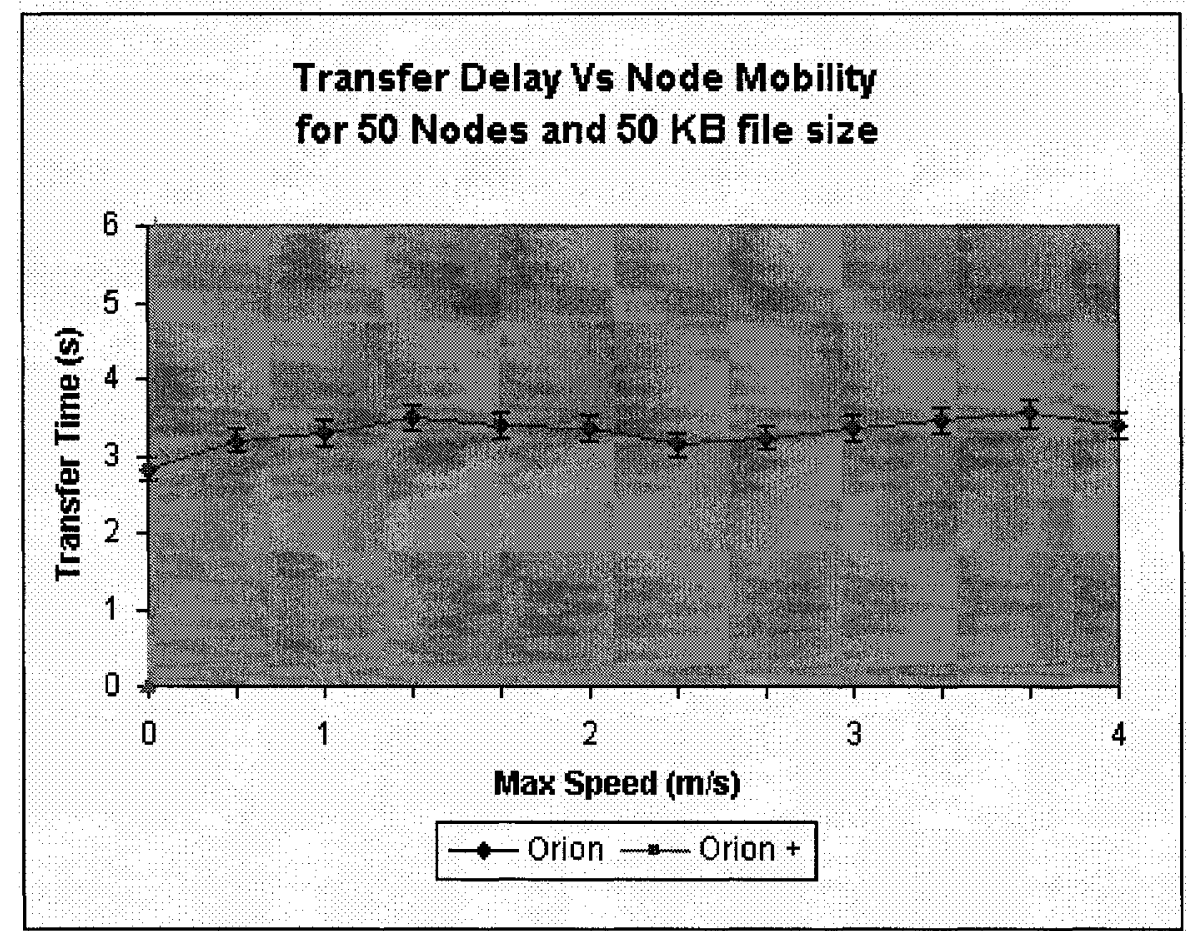

Figure 6.2 Transfer Delay vs. Node Mobility for 50 nodes and $50 \mathrm{~KB}$ file size (Results are displayed with their $95 \% \mathrm{CI}$ )

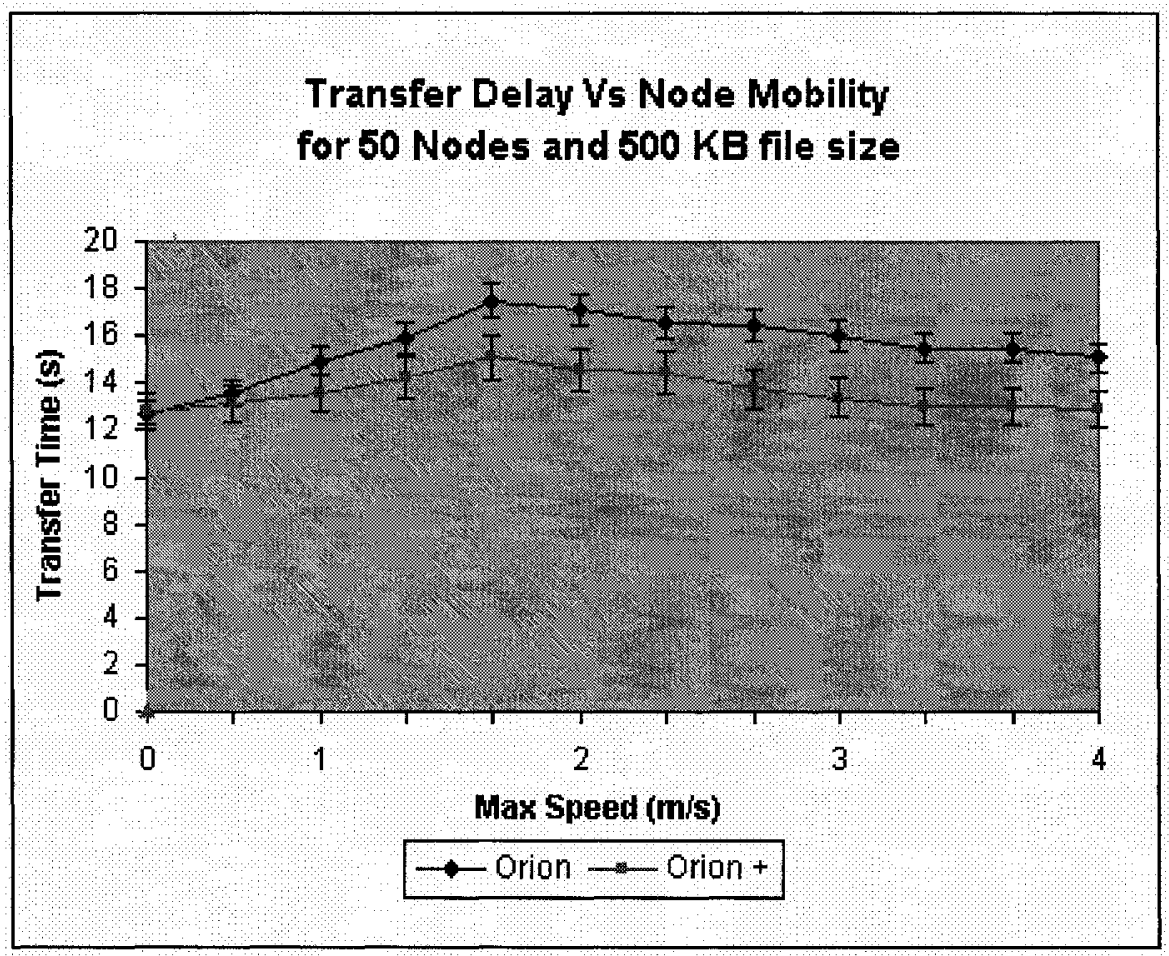

Figure 6.3 Transfer Delay vs. Node Mobility for 50 nodes and $500 \mathrm{~KB}$ file size (Results are displayed with their $95 \% \mathrm{CI}$ ) 


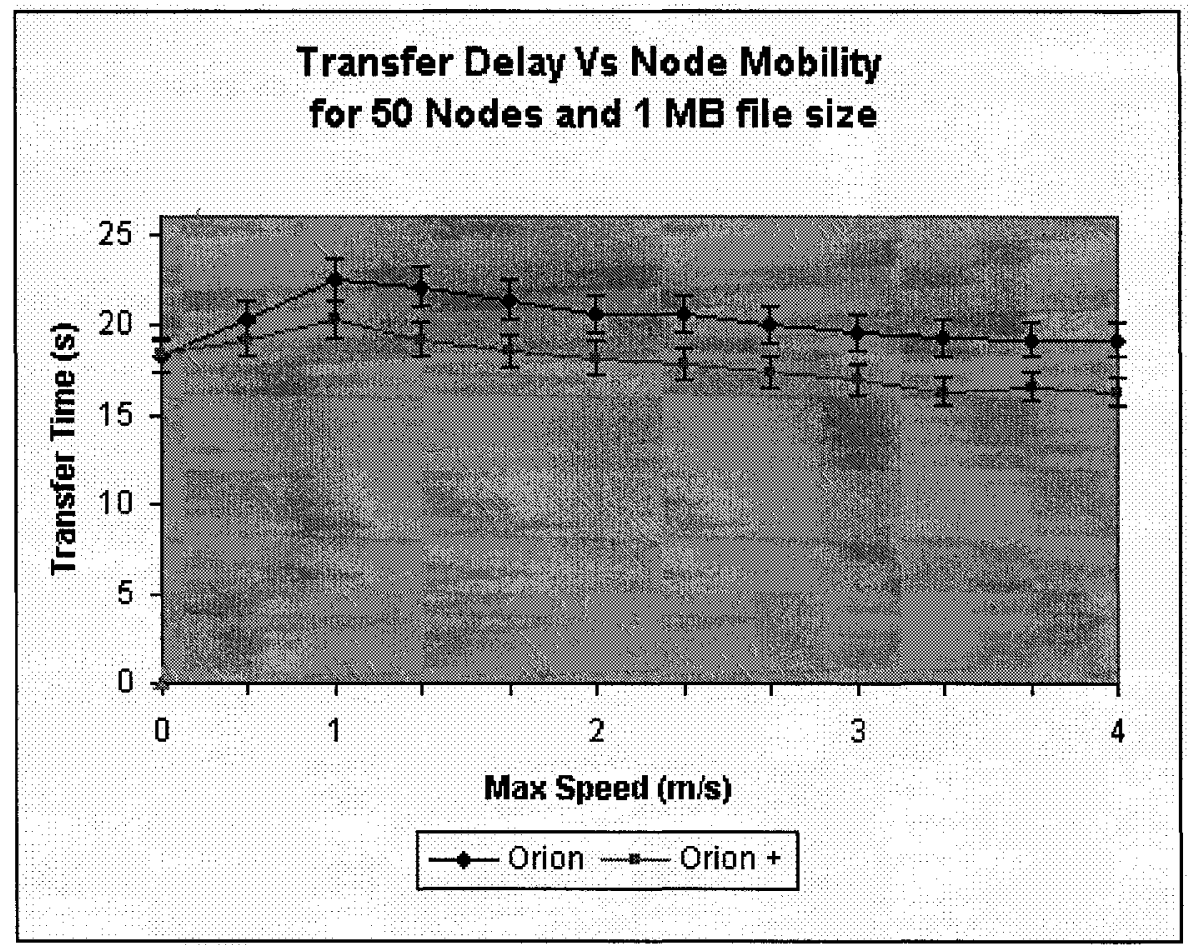

Figure 6.4 Transfer Delay vs. Node Mobility for 50 nodes and $1 \mathrm{MB}$ file size (Results are displayed with their $95 \% \mathrm{CI}$ )

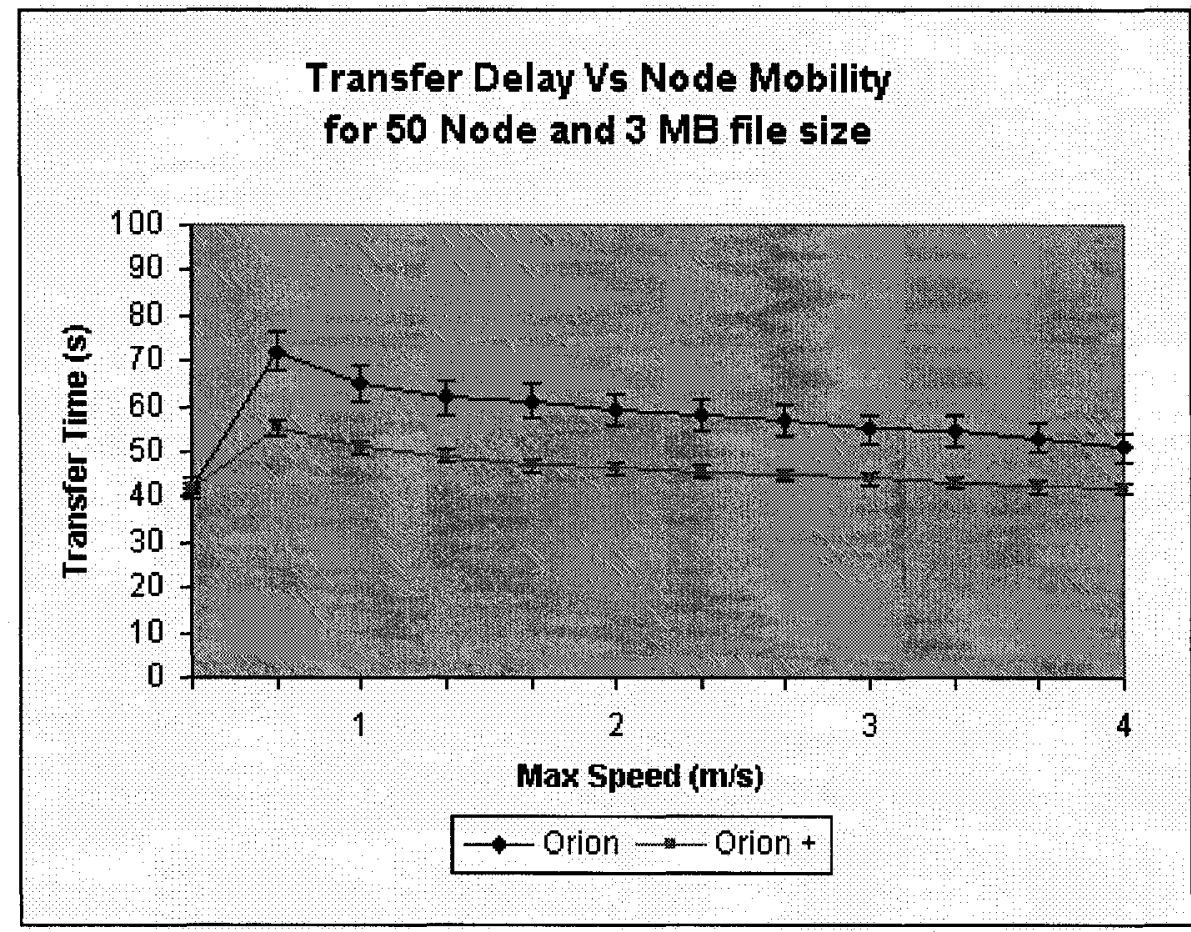

Figure 6.5 Transfer Delay vs. Node Mobility for 50 nodes and $3 \mathrm{MB}$ file size (Results are displayed with their $95 \%$ CI) 


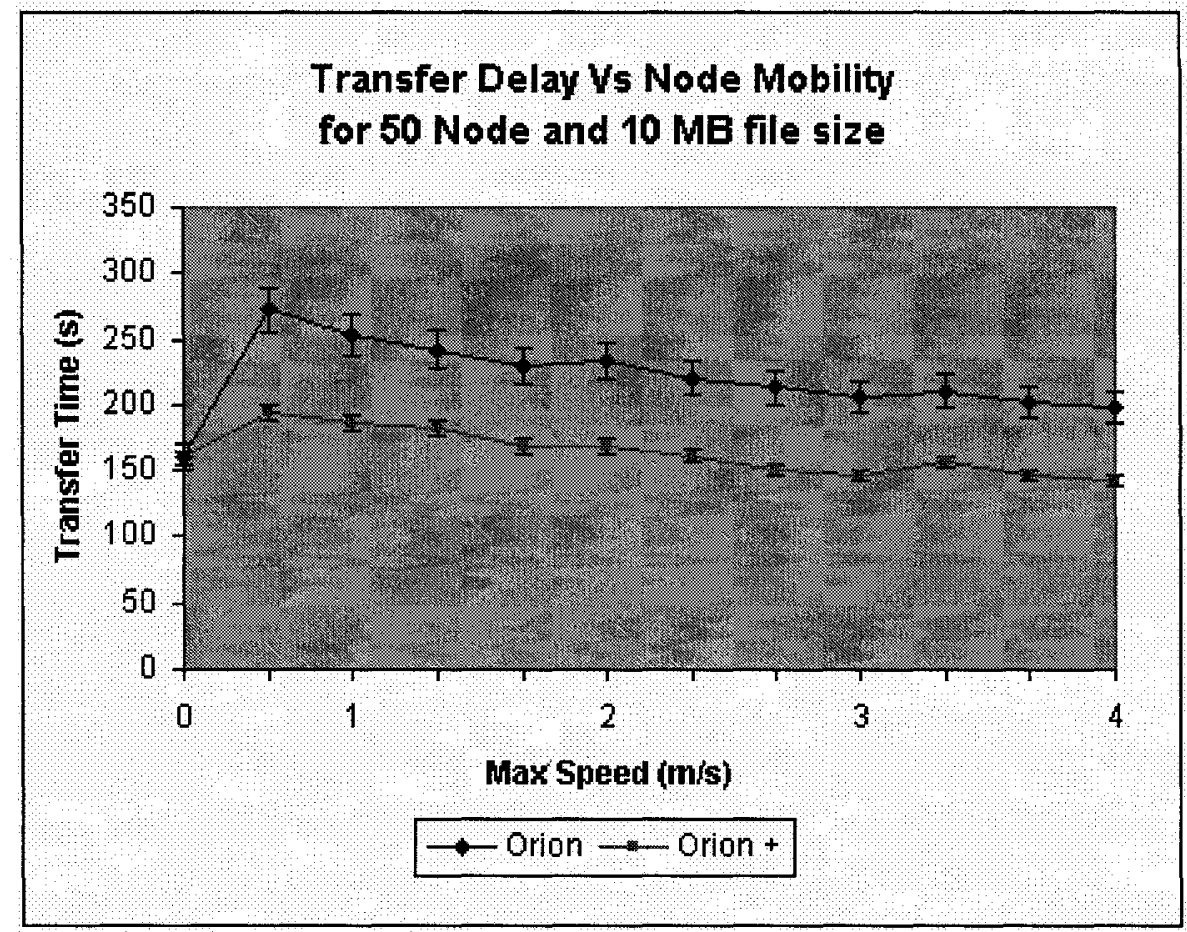

Figure 6.6 Transfer Delay vs. Node Mobility for 50 nodes and $10 \mathrm{MB}$ file size (Results are displayed with their $95 \% \mathrm{CI}$ )

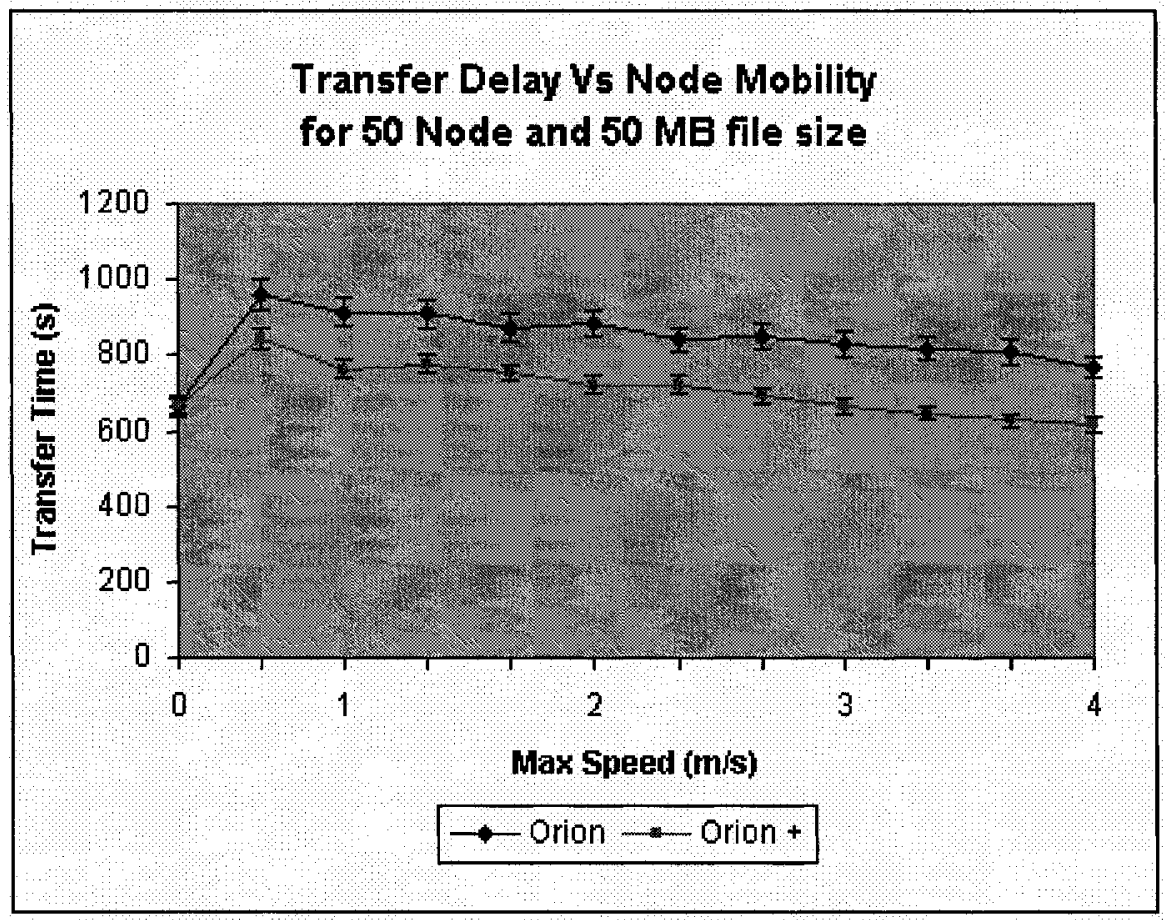

Figure 6.7 Transfer Delay vs. Node Mobility for 50 nodes and 50 MB file size (Results are displayed with their $95 \% \mathrm{CI}$ ) 


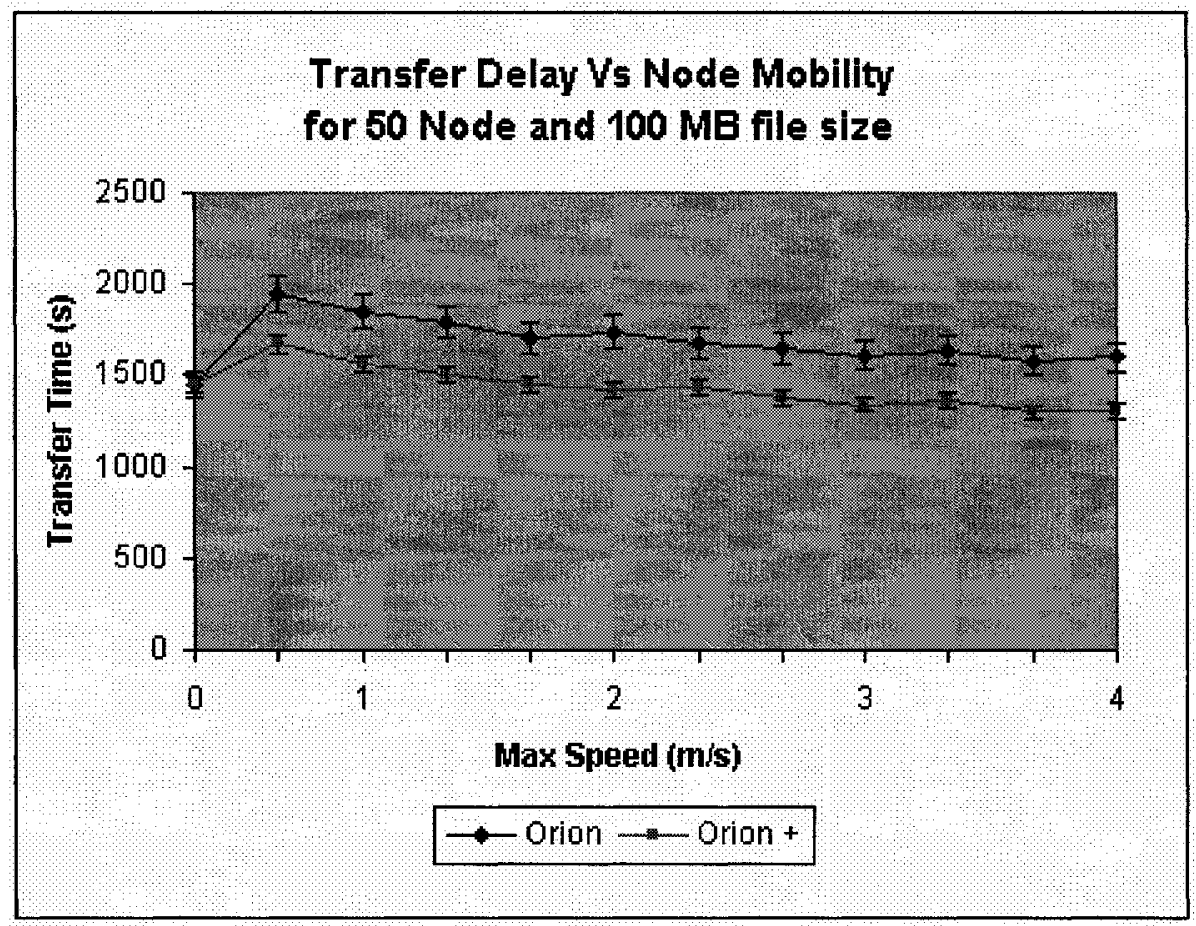

Figure 6.8 Transfer Delay vs. Node Mobility for 50 nodes and $100 \mathrm{MB}$ file size (Results are displayed with their $95 \% \mathrm{CI}$ )

For the high node density scenario (100 nodes network), because of the longer paths used, we see that the transfer times are higher than the 50 nodes scenario. In Figures 6.9 and 6.10, we see that the transfer delay performance of ORION and ORION+ is almost the same because of the small file sizes. In Figure $6.11(500 \mathrm{~KB})$, for low mobility (less than $2 \mathrm{~m} / \mathrm{s}$ max speed), the file is successfully downloaded before there is a need to reflood the network (usage of weekly connected paths result in higher transfer delay). For high mobility, a reflooding is always needed to get new paths (usage of fresh paths decreases the overall transfer delay). In Figure 6.12, we see that the whole file can only be downloaded successfully with no need for reflooding under very low mobility (max speed less than $1 \mathrm{~m} / \mathrm{sec}$ ), for higher max speeds, a reflooding is always required to 
get new paths. For file sizes $3 \mathrm{MB}$ and larger (Figures $6.13-6.16$ ), a reflooding is always required since all paths are broken before the whole file is successfully downloaded.

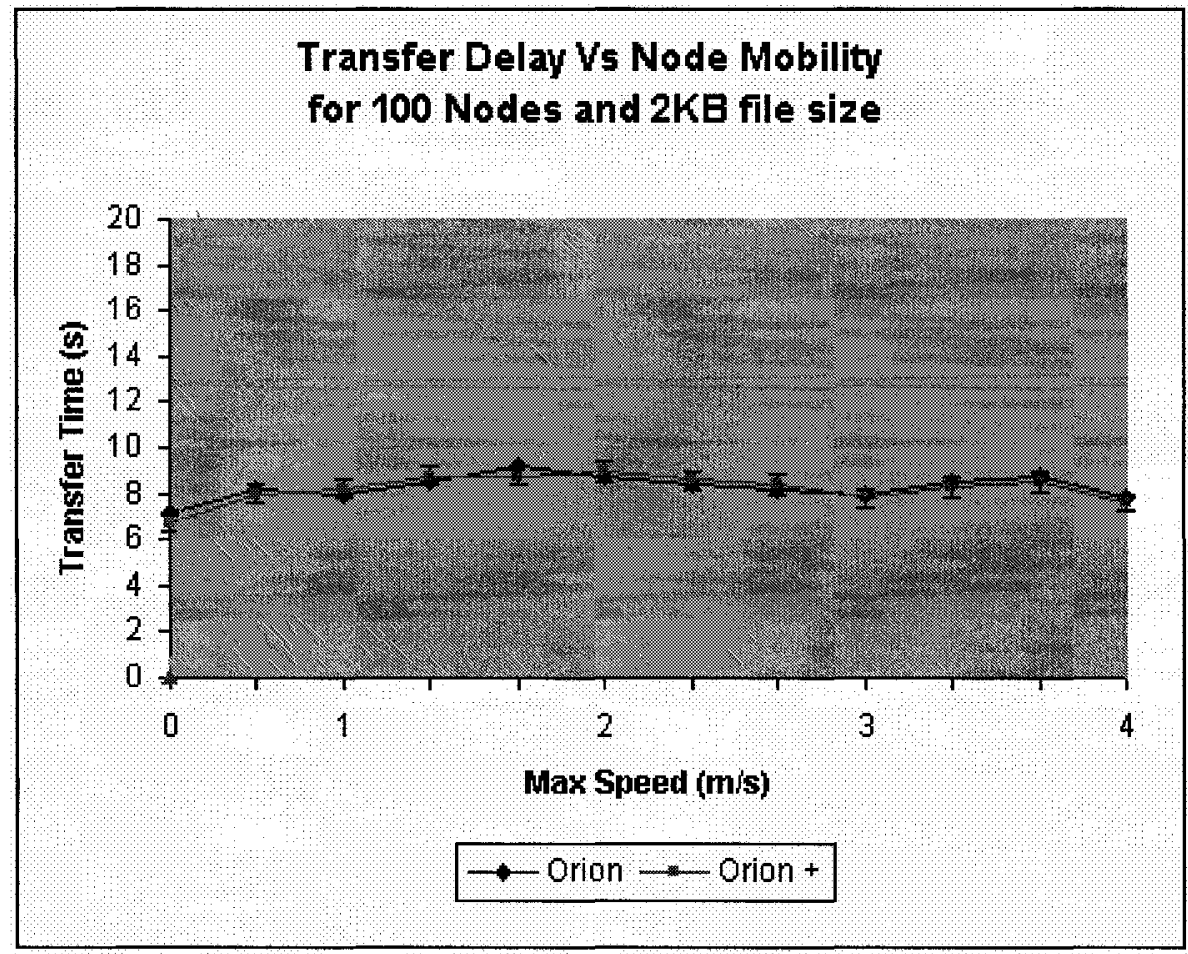

Figure 6.9 Transfer Delay vs. Node Mobility for 100 nodes and 2 KB file size (Results are displayed with their $95 \%$ CI) 


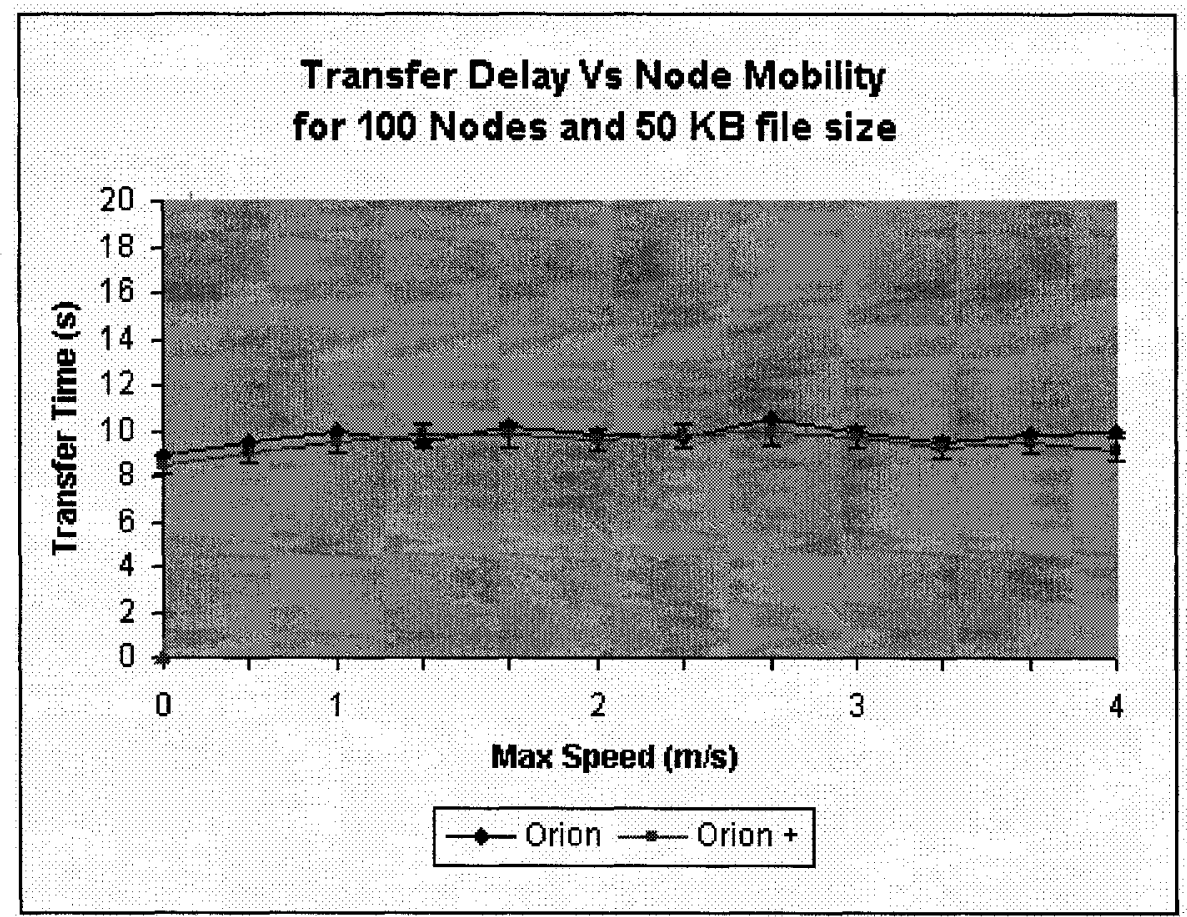

Figure 6.10 Transfer Delay vs. Node Mobility for 100 nodes and 50 KB file size (Results are displayed with their $95 \% \mathrm{CI}$ )

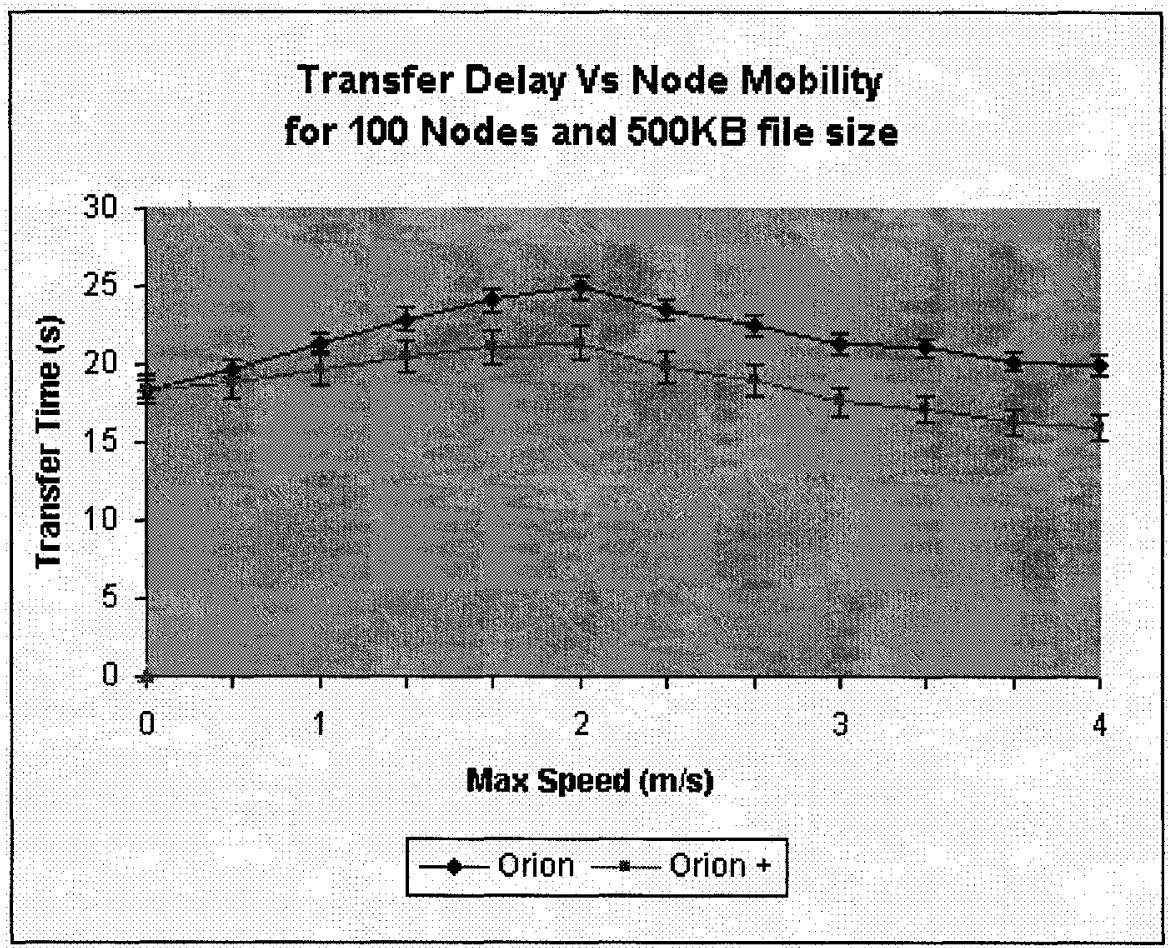

Figure 6.11 Transfer Delay vs. Node Mobility for 100 nodes and $500 \mathrm{~KB}$ file size (Results are displayed with their $95 \%$ CI) 


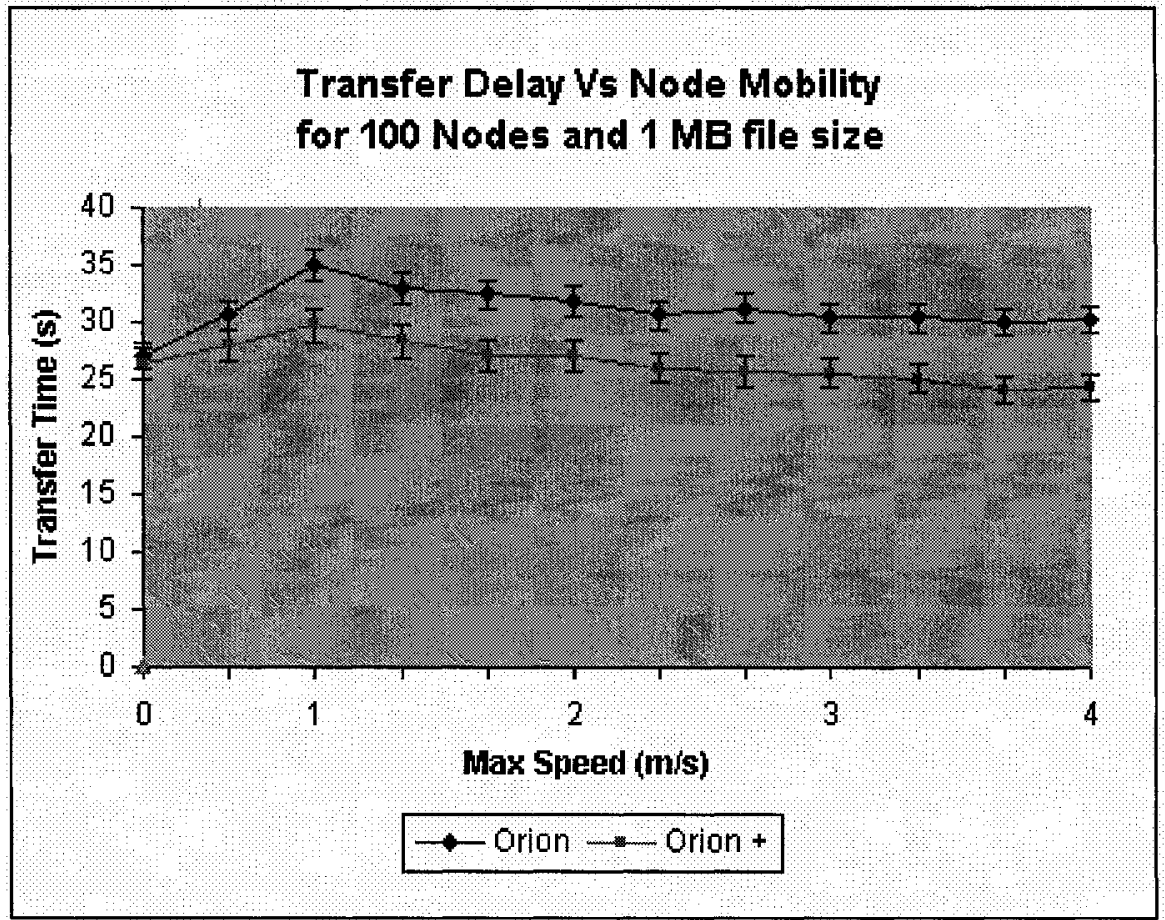

Figure 6.12 Transfer Delay vs. Node Mobility for 100 nodes and $1 \mathrm{MB}$ file size (Results are displayed with their $95 \%$ CI)

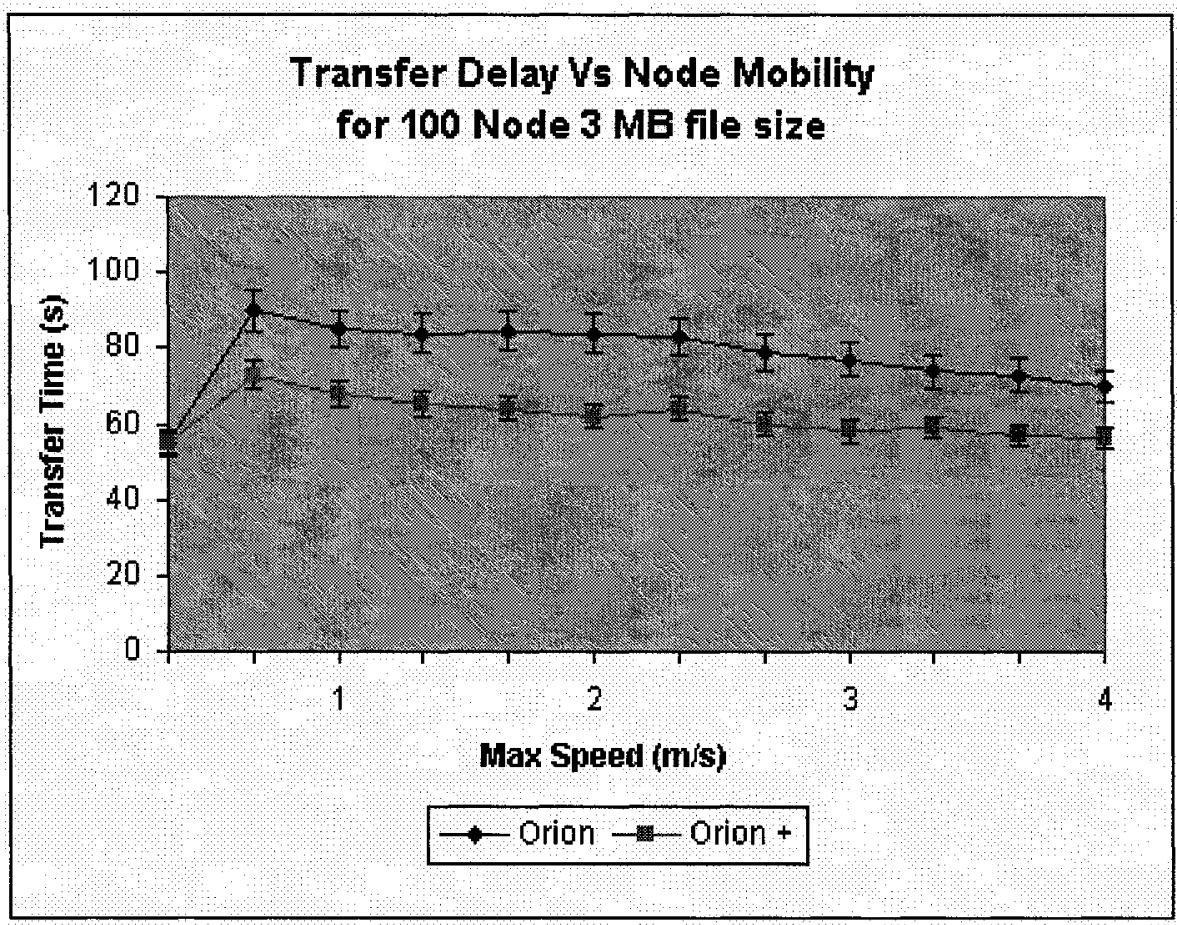

Figure 6.13 Transfer Delay vs. Node Mobility for 100 nodes and $3 \mathrm{MB}$ file size (Results are displayed with their $95 \% \mathrm{CI}$ ) 


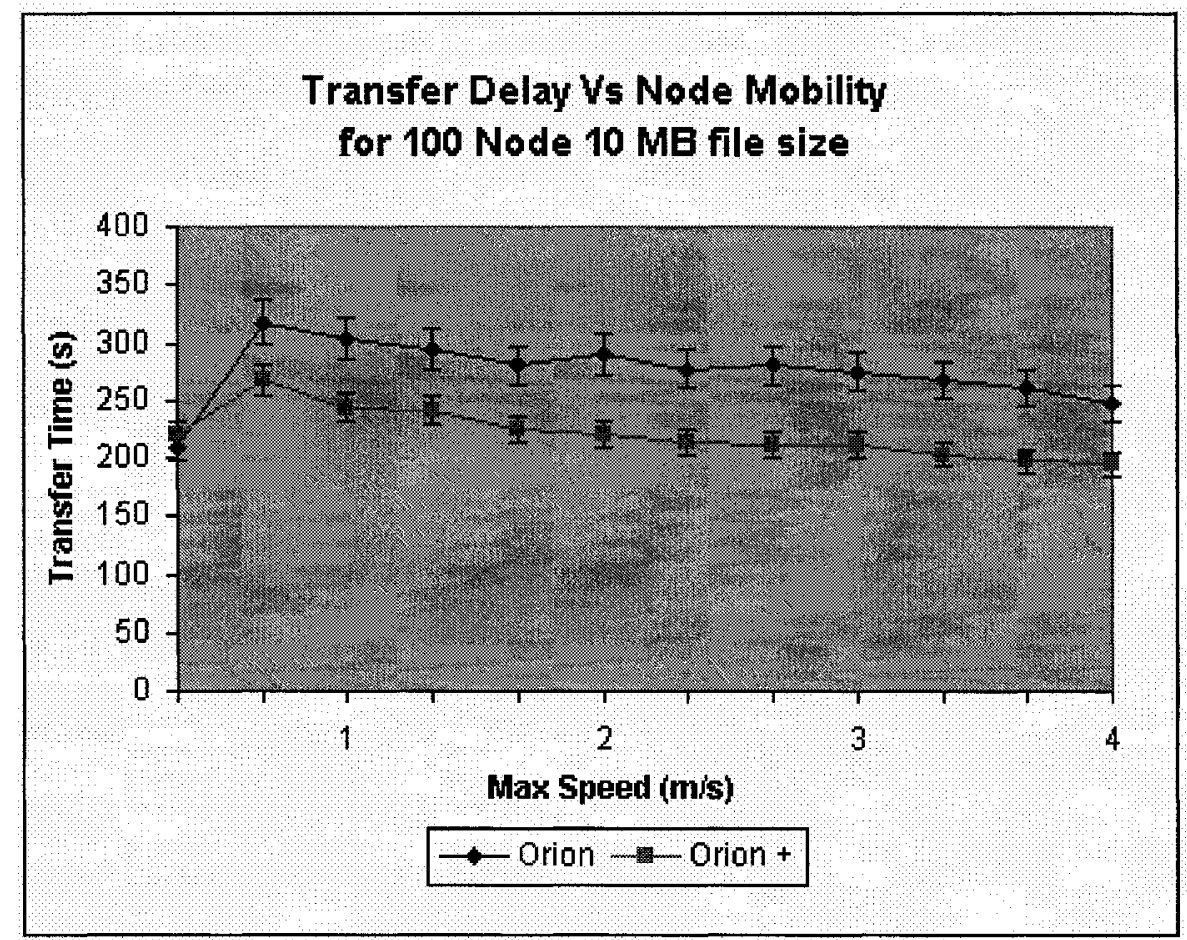

Figure 6.14 Transfer Delay vs. Node Mobility for 100 nodes and $10 \mathrm{MB}$ file size (Results are displayed with their $95 \% \mathrm{CI}$ )

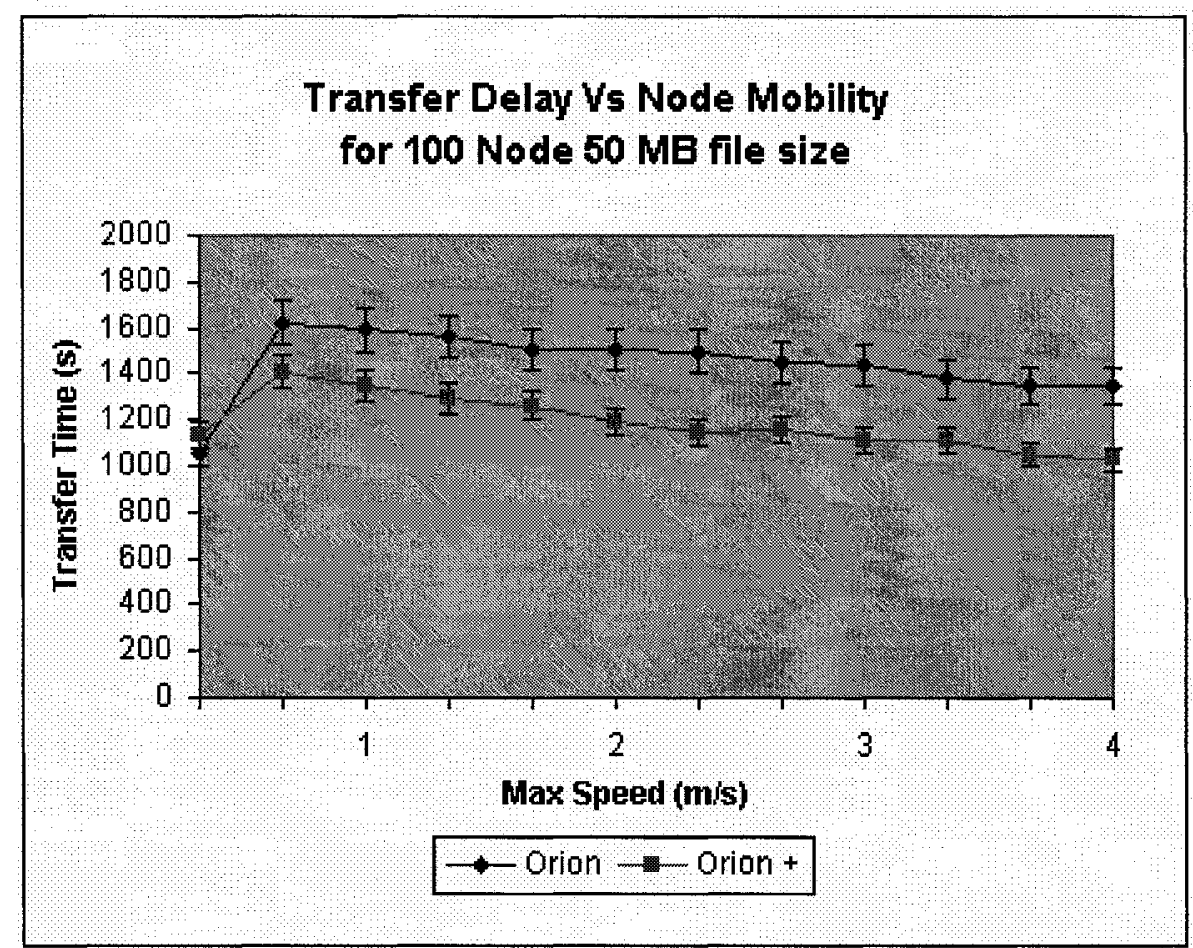

Figure 6.15 Transfer Delay vs. Node Mobility for 100 nodes and $50 \mathrm{MB}$ file size (Results are displayed with their $95 \% \mathrm{CI}$ ) 


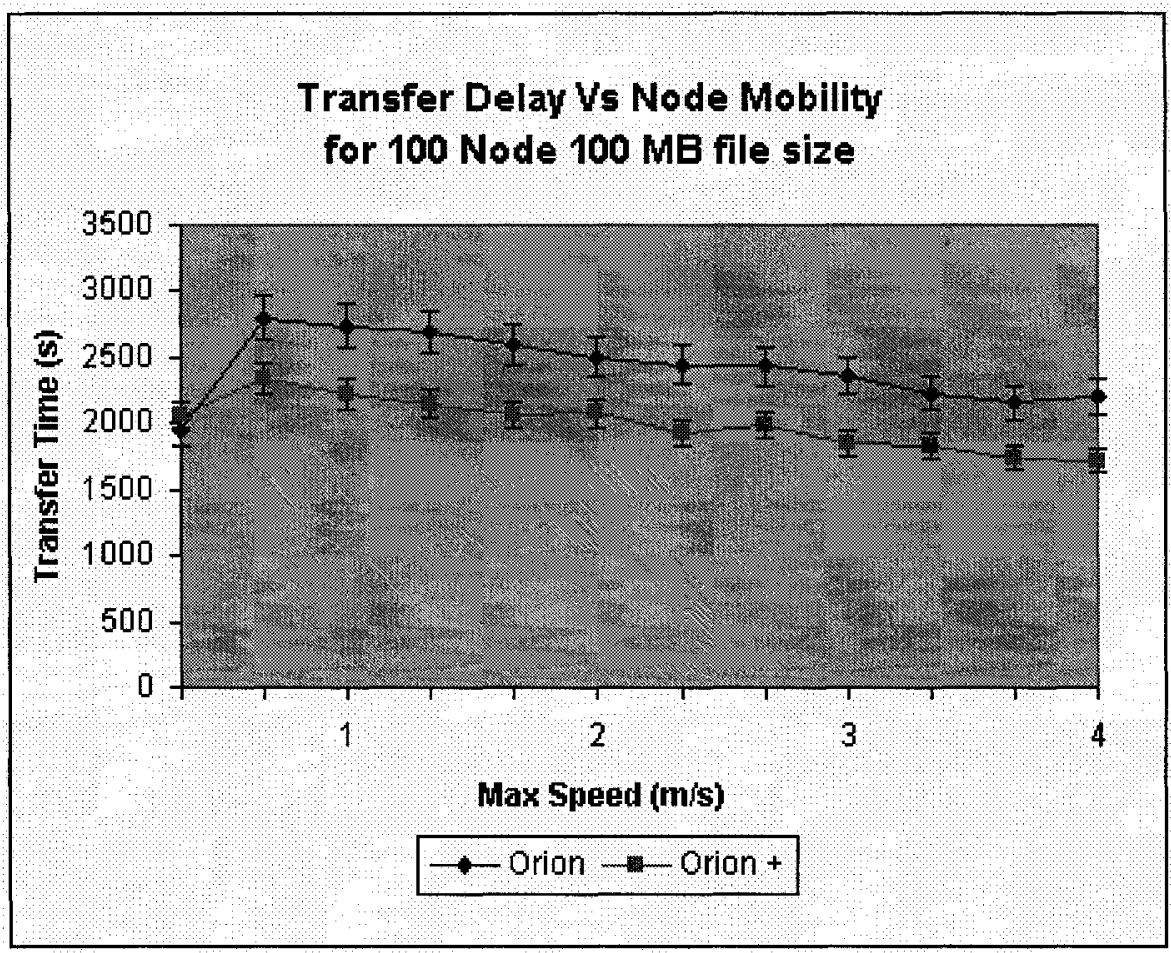

Figure 6.16 Transfer Delay vs. Node Mobility for 100 nodes and $100 \mathrm{MB}$ file size (Results are displayed with their $95 \%$ CI)

\subsubsection{Successful file transfer}

Successful file transfer is a very important attribute from the end-user's point of view because unless the whole file is downloaded successfully, it cannot be used by the user. As it was defined in [2], the download for ORION is considered "failed" if the requesting node runs out of alternative paths after a single re-query. Figures 6.17 to 6.24 show the simulation results for the 50 nodes scenario and Figures 6.25 to 6.32 show the simulation results for the 100 nodes scenario for different file sizes. Here we can see that ORION+ is able to complete the download process of more files before it runs out of next hops. This is mainly attributed to the better choice of routes while they exist instead of using a specific route until it breaks. We also see that due to the better connectivity of the 100 nodes scenario, ORION+ was able to achieve greater percentage of successful 
transfers compared to the 50 nodes scenario. We can see that both of ORION and ORION+ are always able to achieve successful downloading for small file sizes (2 KB and $50 \mathrm{~KB}$ ) before they run out of alternative routes. As we increase the file size, we can see that both of ORION and ORION+ run out of alternative paths more quickly especially at high mobility conditions. Under no mobility conditions, the success rate for both of ORION and ORION+ are the same due to the same connectivity condition for both. For large files, more than one re-query is always required to complete the download.

In Figures 6.17 and 6.18 , because of the very small file sizes, both of ORION and ORION+ are able to successfully download all the files. Under no mobility, only the subset of reachable files could be downloaded. For the $500 \mathrm{~KB}$ file size (Figure 6.19), we see that ORION+ is able to maintain higher percentage of successful file transfers under high mobility compared with ORION. The percentage of successful file transfers slowly decreases for both of ORION and ORION+ when increasing the file sizes (Figures 6.20 6.24) since the set of available paths are unable to complete the file transfers before all paths are broken. In the 100 nodes scenario (Figures $6.25-6.32$ ), we see that because of better network connectivity, both of ORION and ORION+ were able to achieve higher percentage of successful file transfers than the 50 nodes scenario for the same file sizes. 


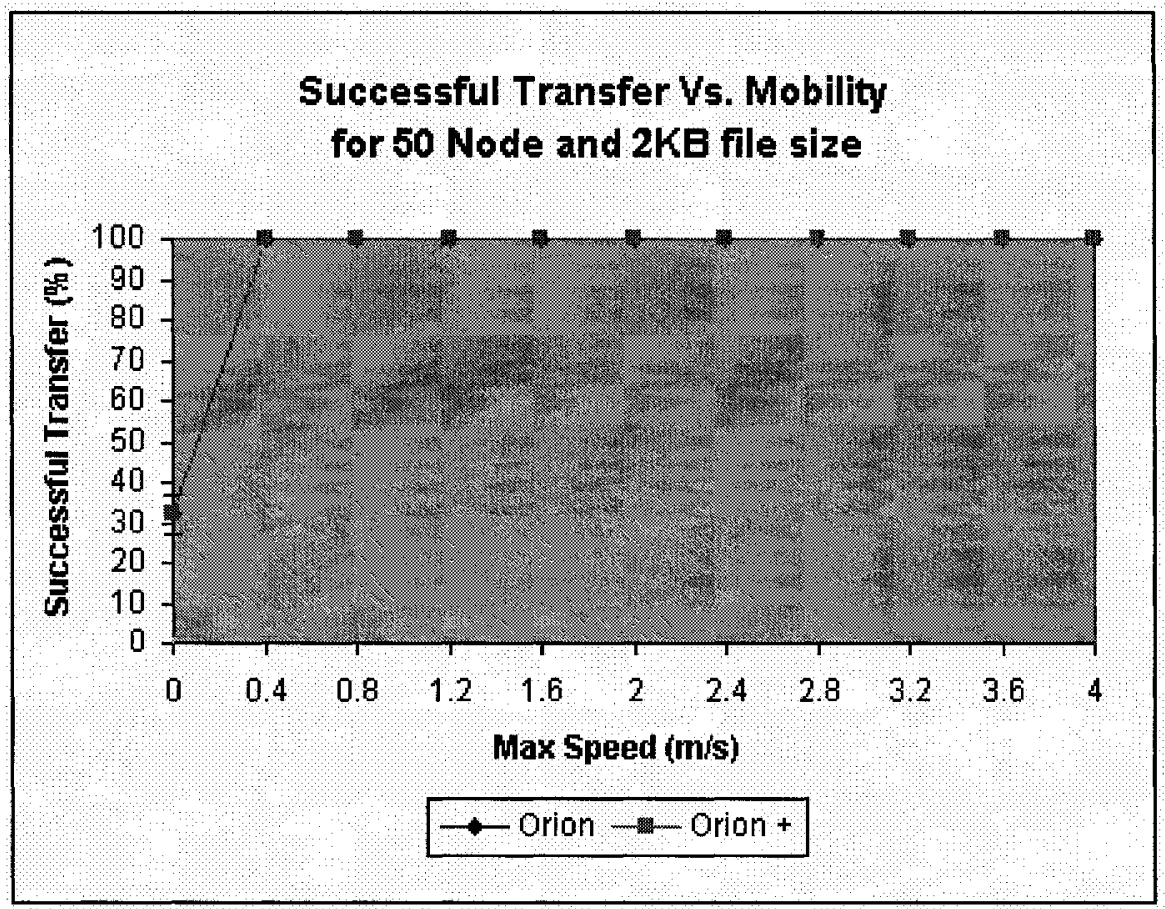

Figure 6.17 Successful Transfer vs. Node Mobility for 50 nodes and 2 KB file size (Results are displayed with their $95 \%$ CI)

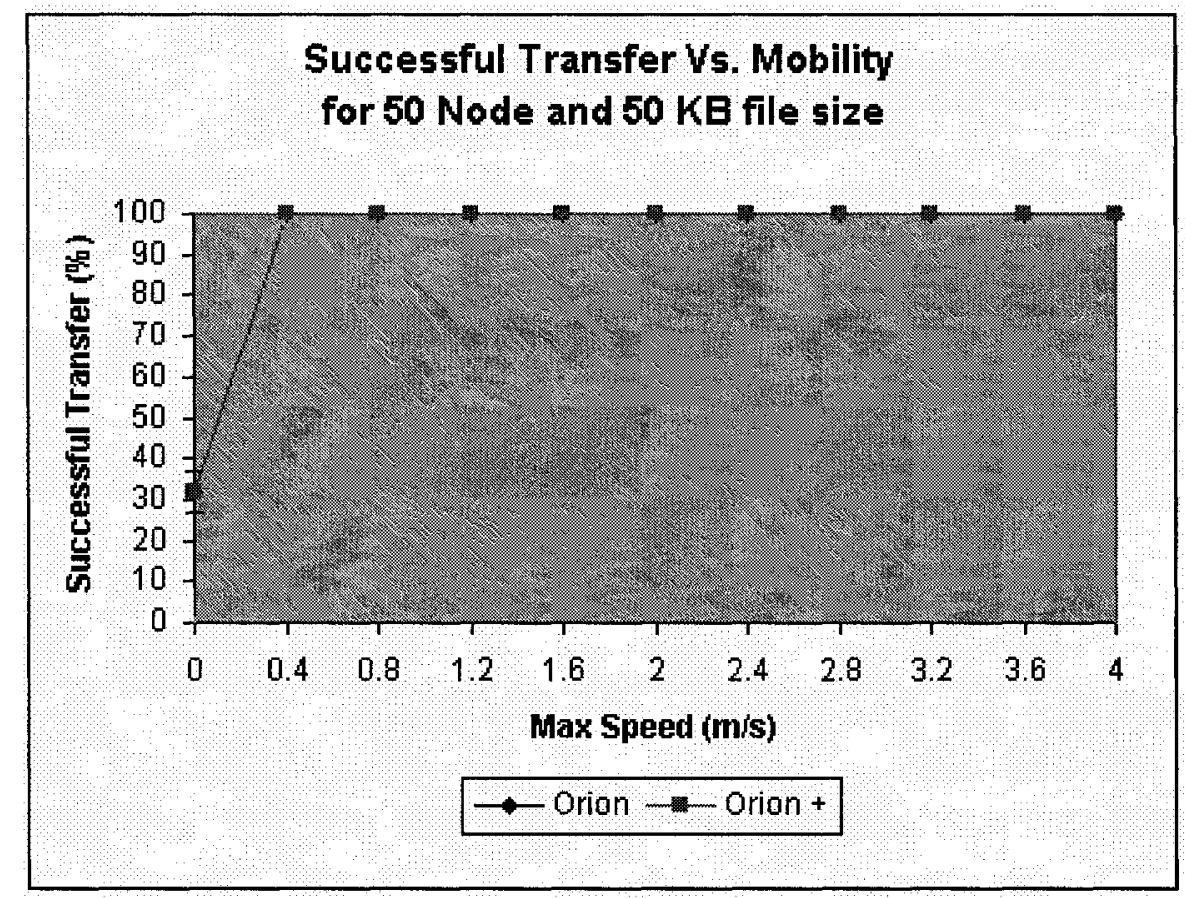

Figure 6.18 Successful Transfer vs. Node Mobility for 50 nodes and 50 KB file size (Results are displayed with their $95 \% \mathrm{CI}$ ) 


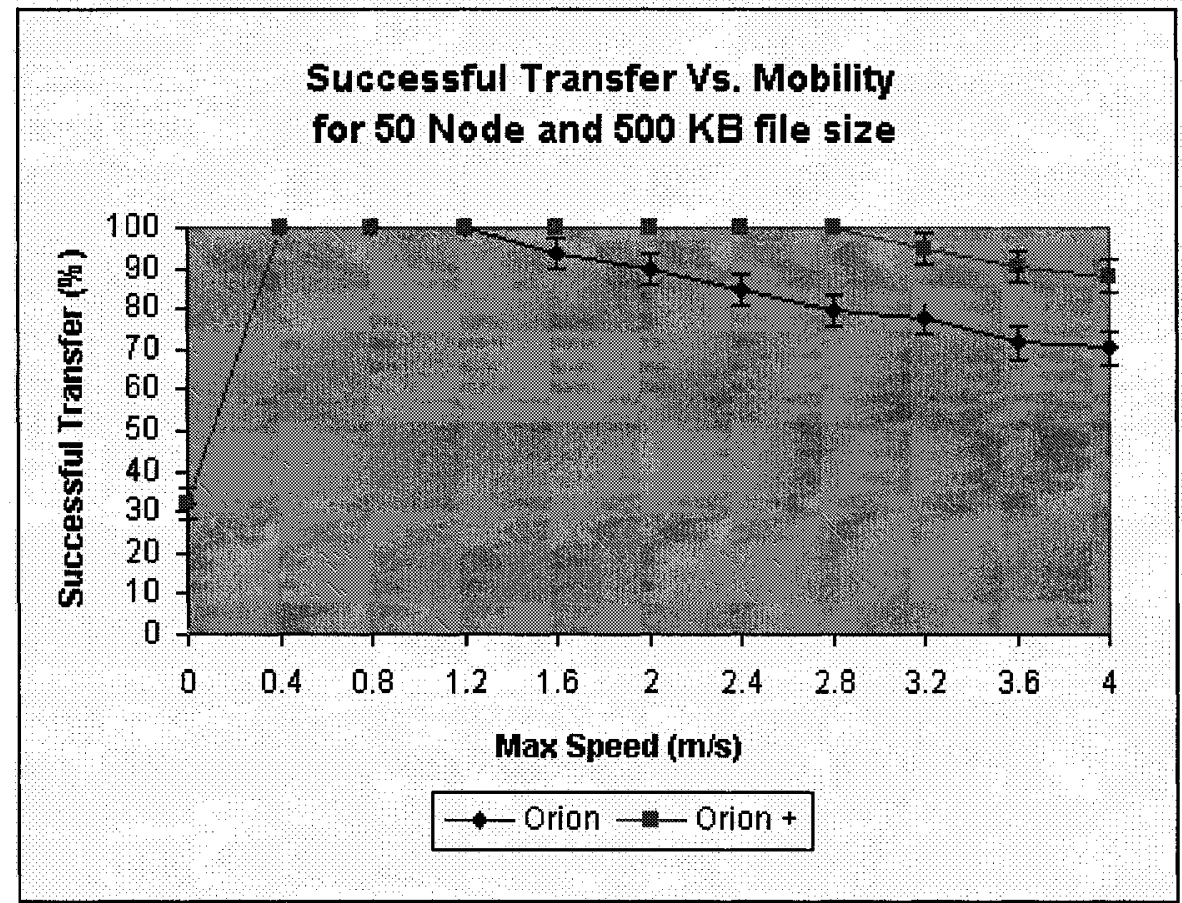

Figure 6.19 Successful Transfer vs. Node Mobility for 50 nodes and $500 \mathrm{~KB}$ file size (Results are displayed with their $95 \% \mathrm{CI}$ )

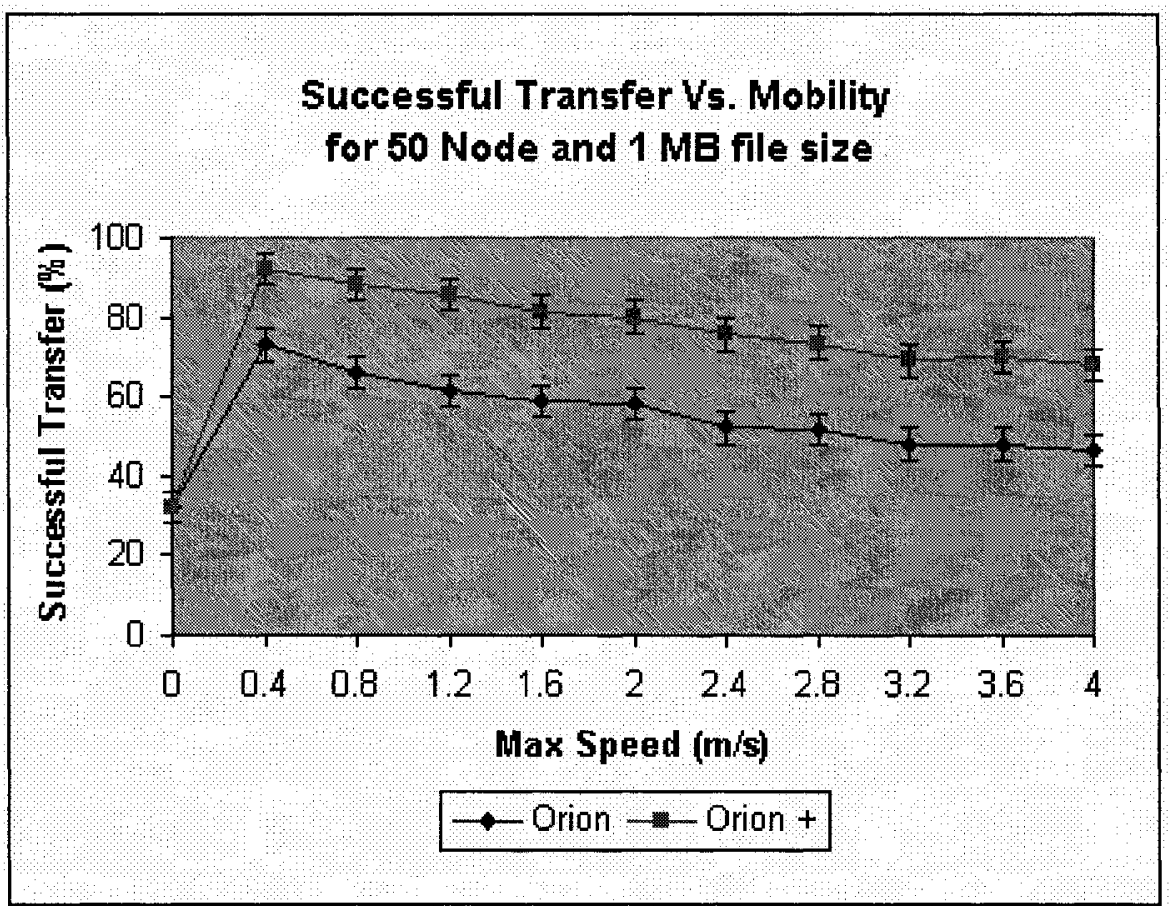

Figure 6.20 Successful Transfer vs. Node Mobility for 50 nodes and 1 MB file size (Results are displayed with their $95 \% \mathrm{CI}$ ) 


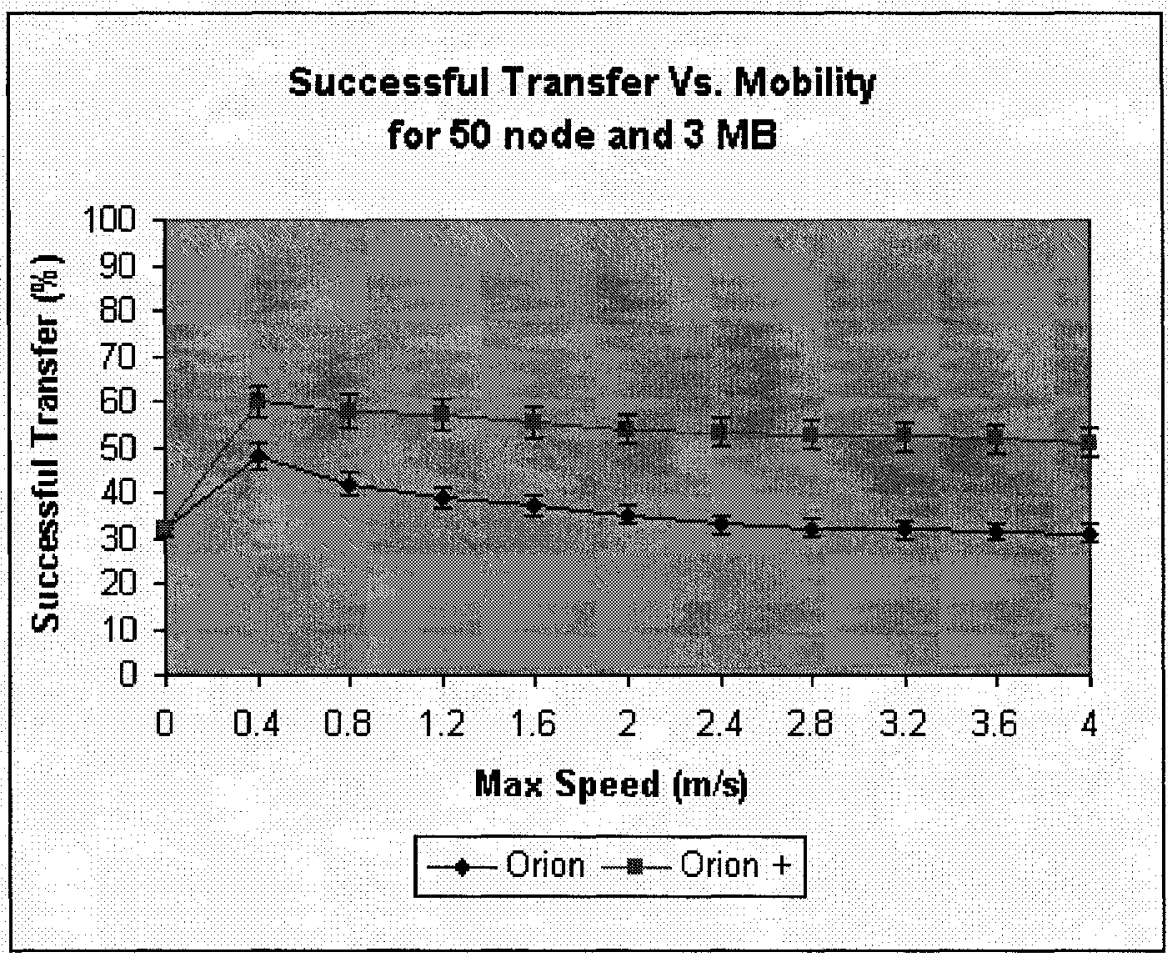

Figure 6.21 Successful Transfer vs. Node Mobility for 50 nodes and 3 MB file size (Results are displayed with their $95 \% \mathrm{CI}$ )

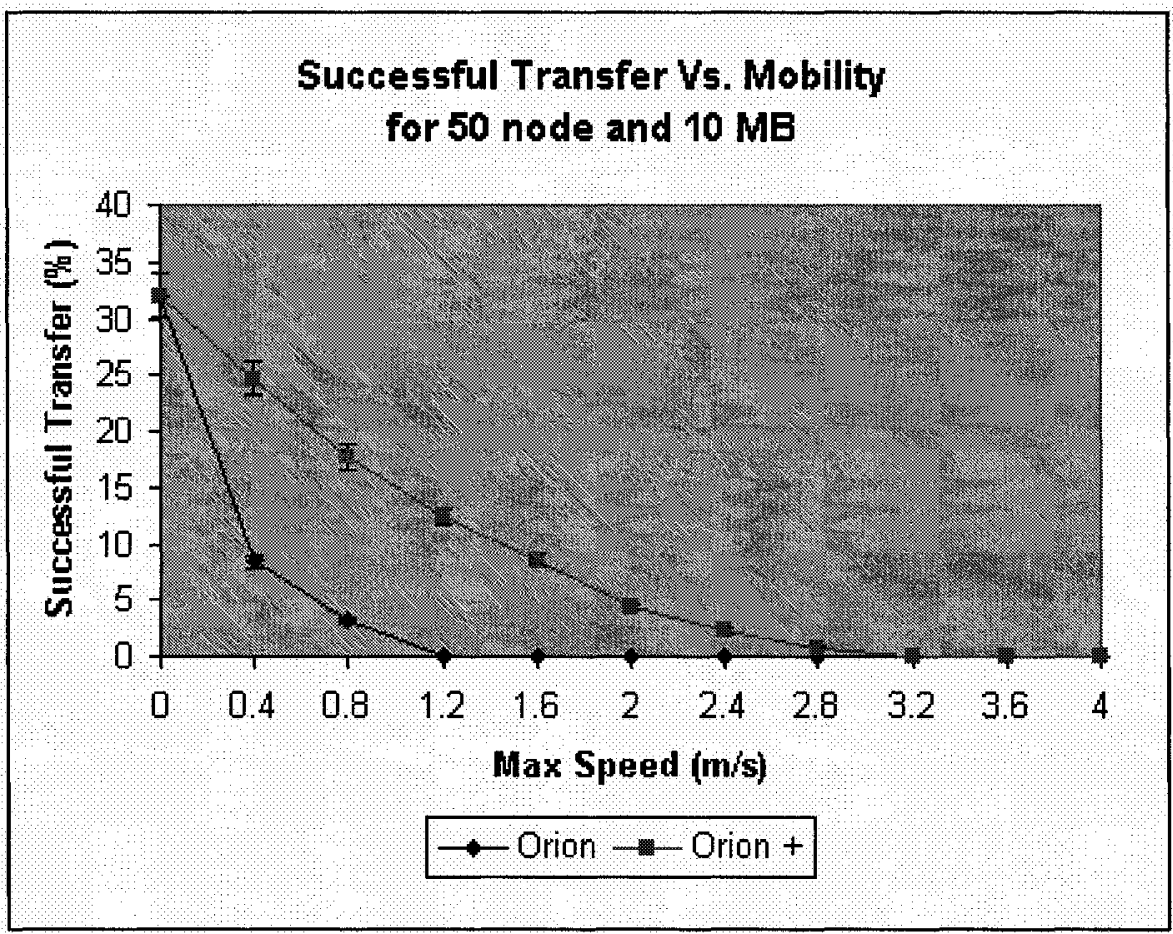

Figure 6.22 Successful Transfer vs. Node Mobility for 50 nodes and $10 \mathrm{MB}$ file size (Results are displayed with their $95 \% \mathrm{CI}$ ) 


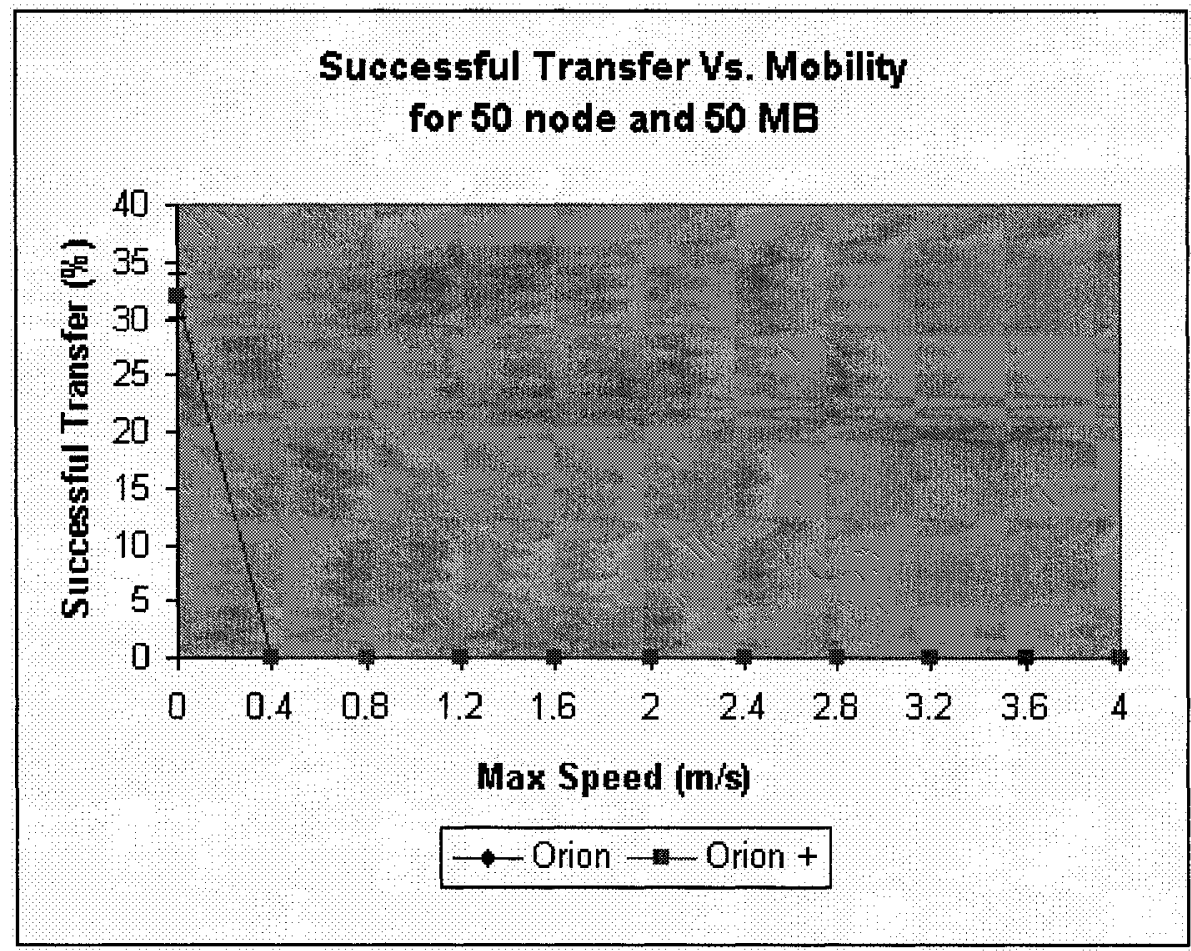

Figure 6.23 Successful Transfer vs. Node Mobility for 50 nodes and $50 \mathrm{MB}$ file size (Results are displayed with their $95 \% \mathrm{CI}$ )

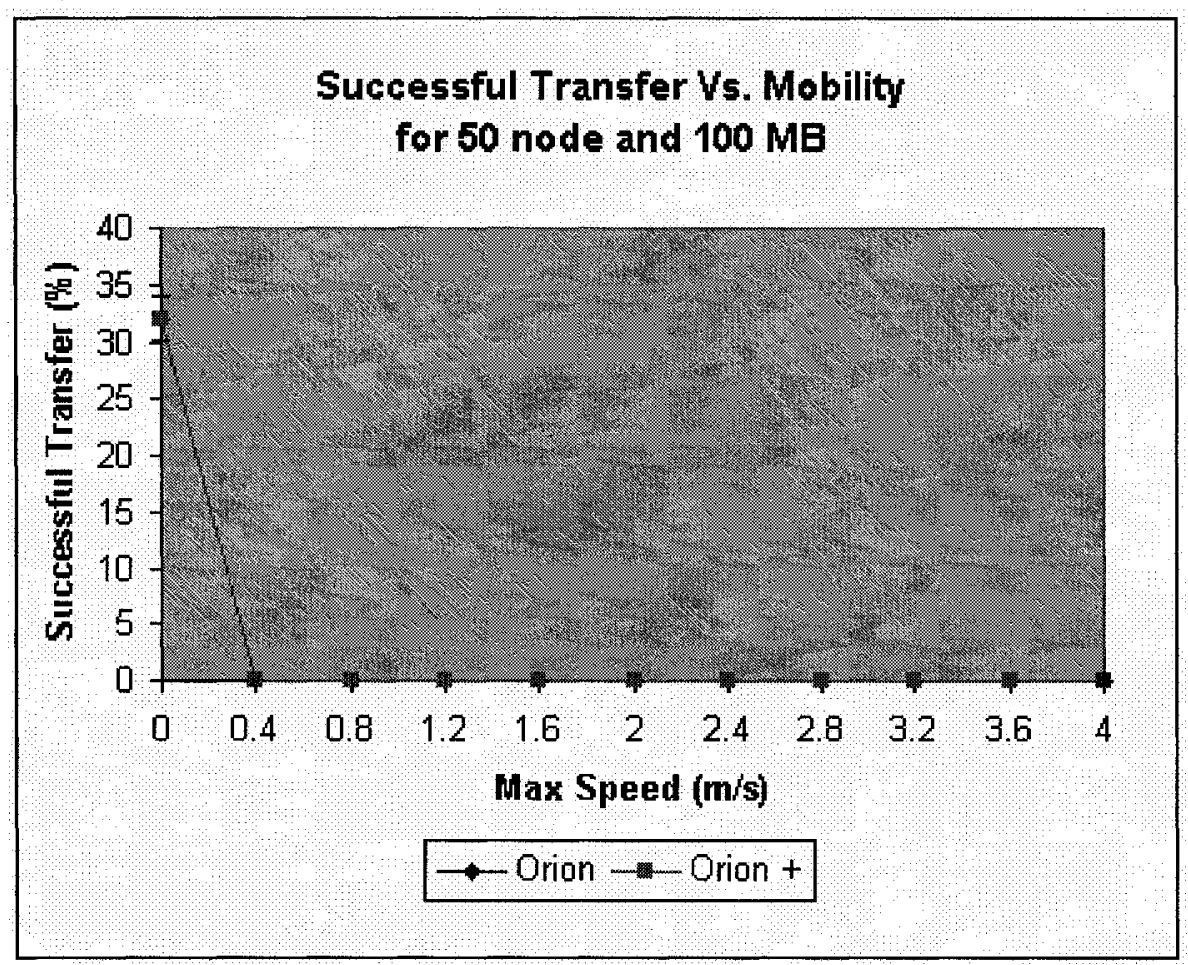

Figure 6.24 Successful Transfer vs. Node Mobility for 50 nodes and $100 \mathrm{MB}$ file size (Results are displayed with their $95 \% \mathrm{CI}$ ) 


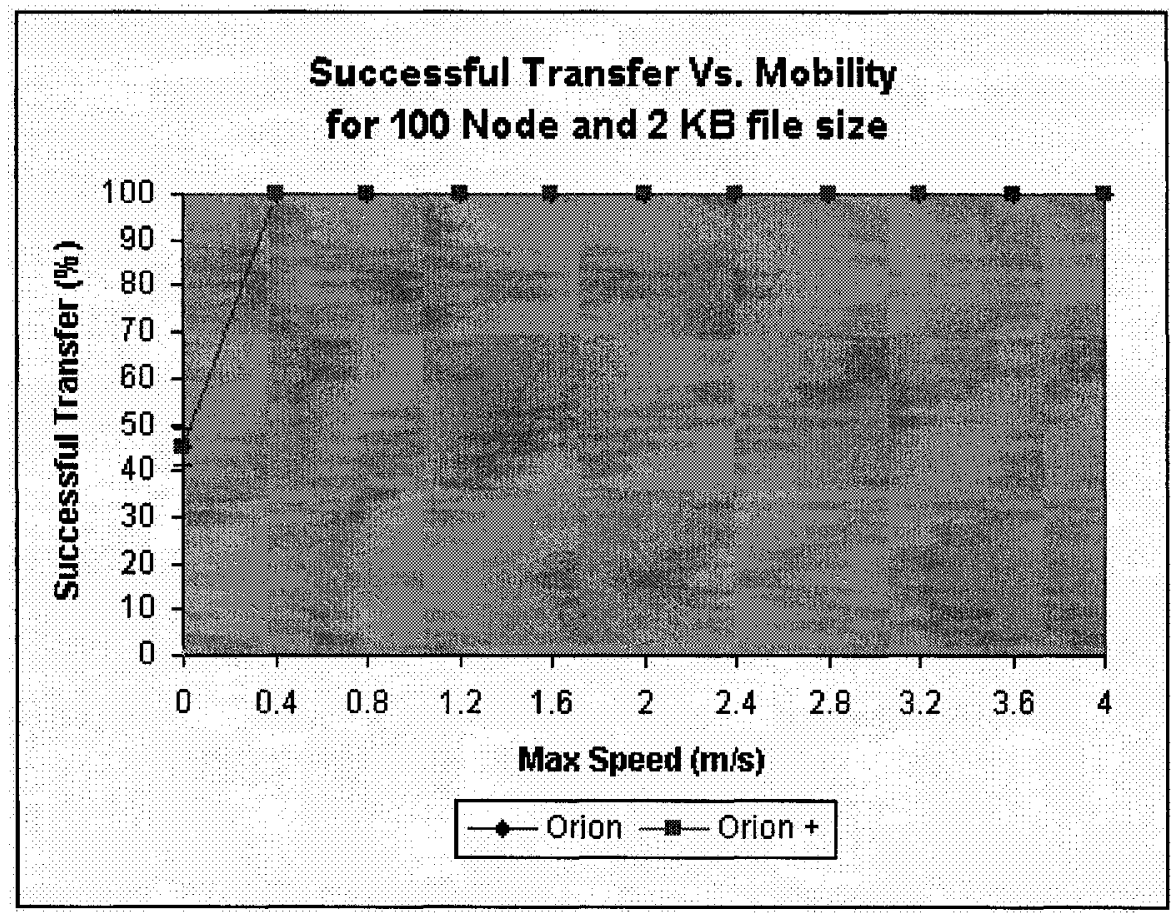

Figure 6.25 Successful Transfer vs. Node Mobility for 100 nodes and 2 KB file size (Results are displayed with their $95 \%$ CI)

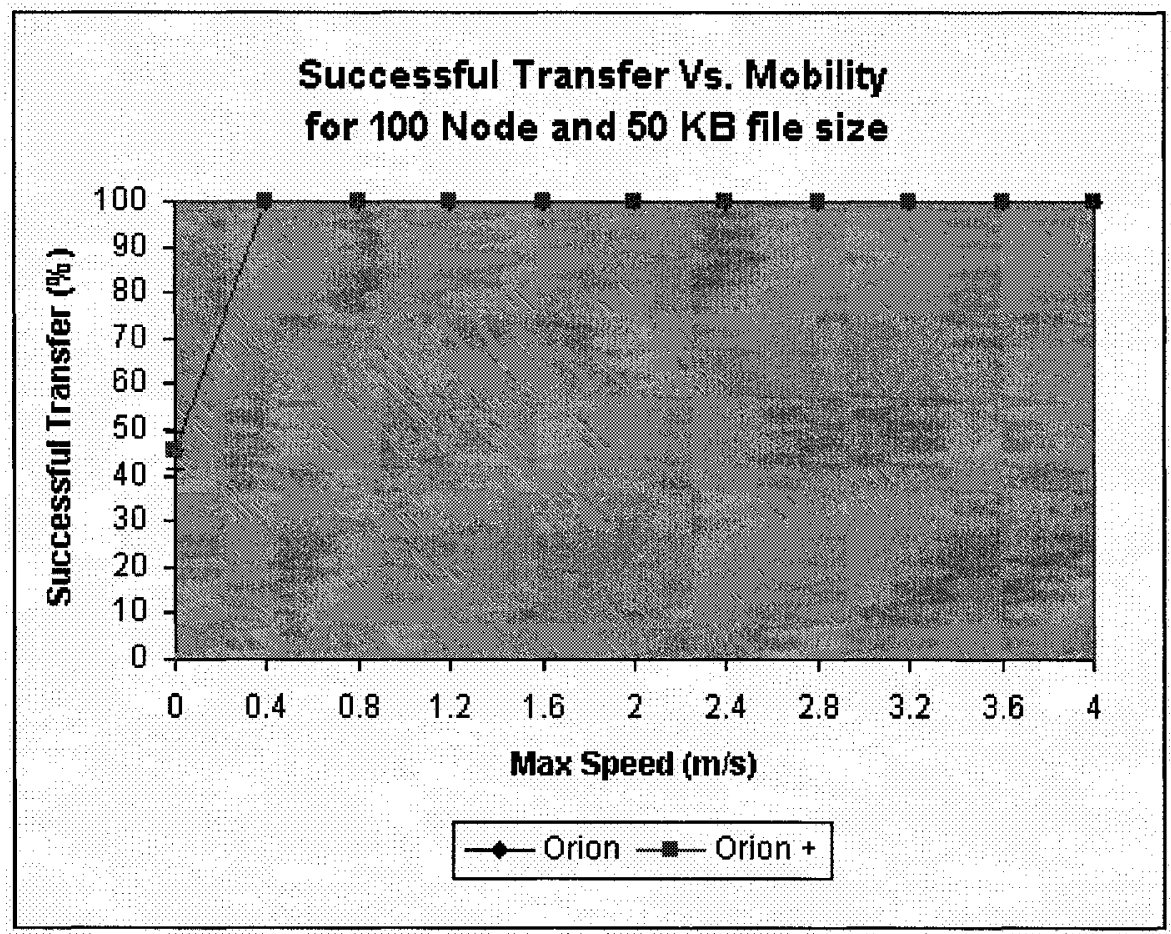

Figure 6.26 Successful Transfer vs. Node Mobility for 100 nodes and $50 \mathrm{~KB}$ file size (Results are displayed with their $95 \% \mathrm{CI}$ ) 


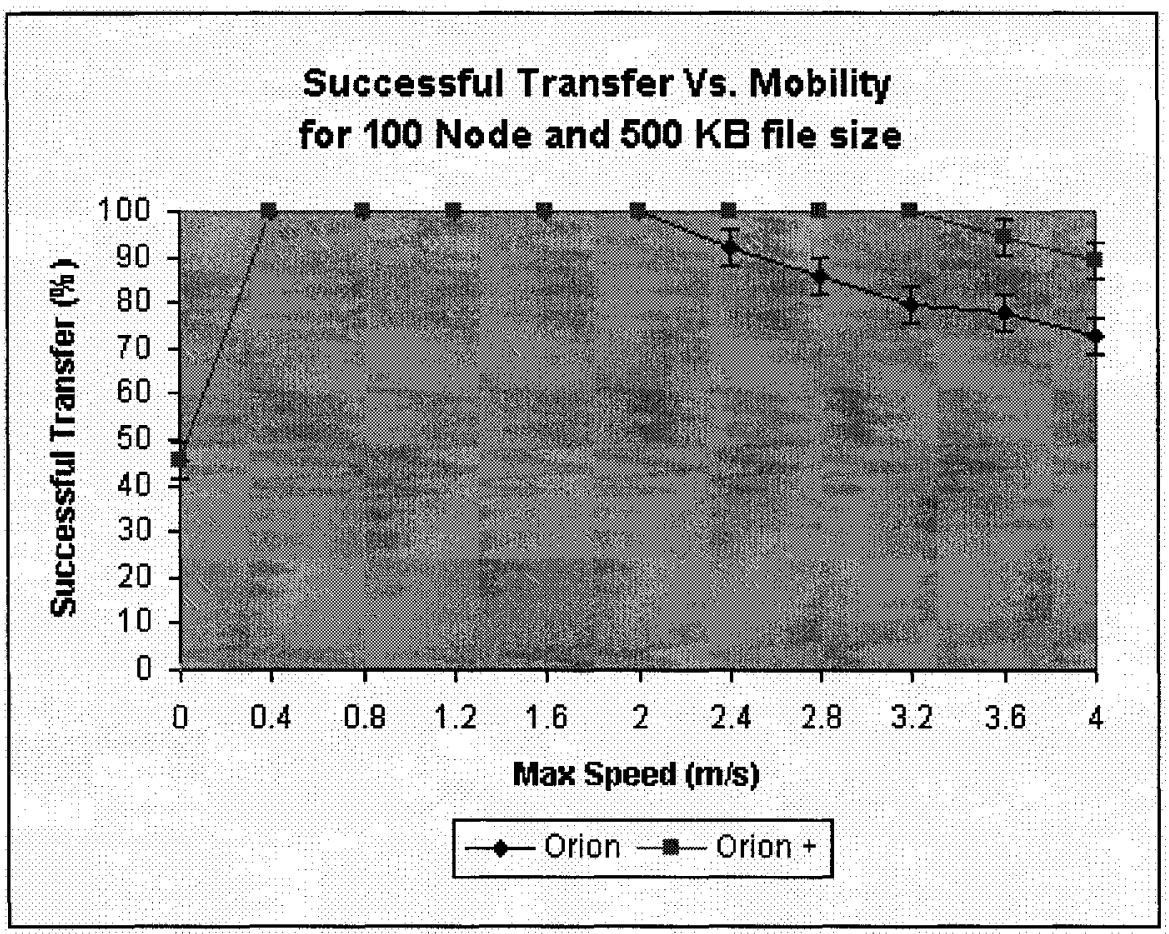

Figure 6.27 Successful Transfer vs. Node Mobility for 100 nodes and 500 KB file size (Results are displayed with their $95 \% \mathrm{CI}$ )

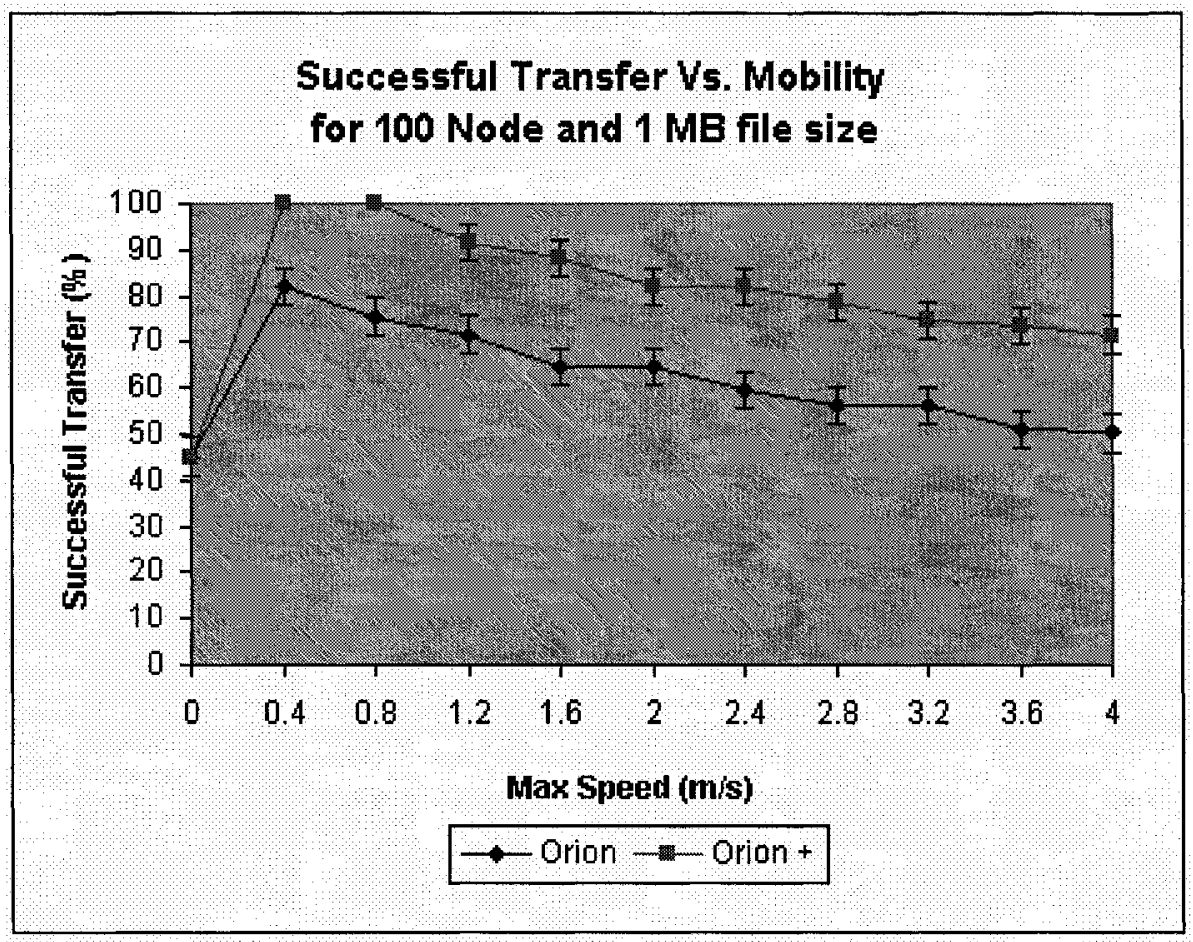

Figure 6.28 Successful Transfer vs. Node Mobility for 100 nodes and $1 \mathrm{MB}$ file size (Results are displayed with their $95 \%$ CI) 


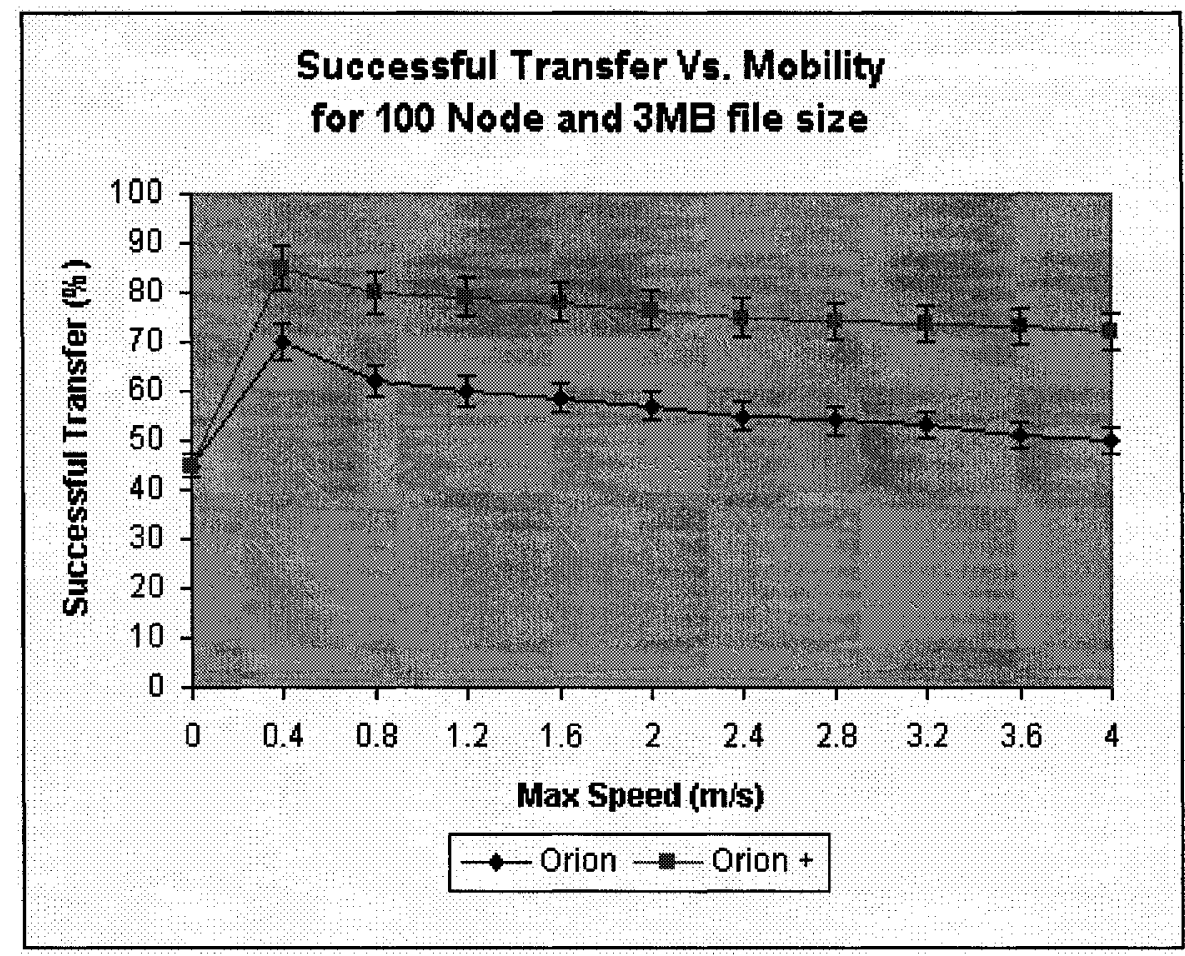

Figure 6.29 Successful Transfer vs. Node Mobility for 100 nodes and $3 \mathrm{MB}$ file size (Results are displayed with their $95 \% \mathrm{CI}$ )

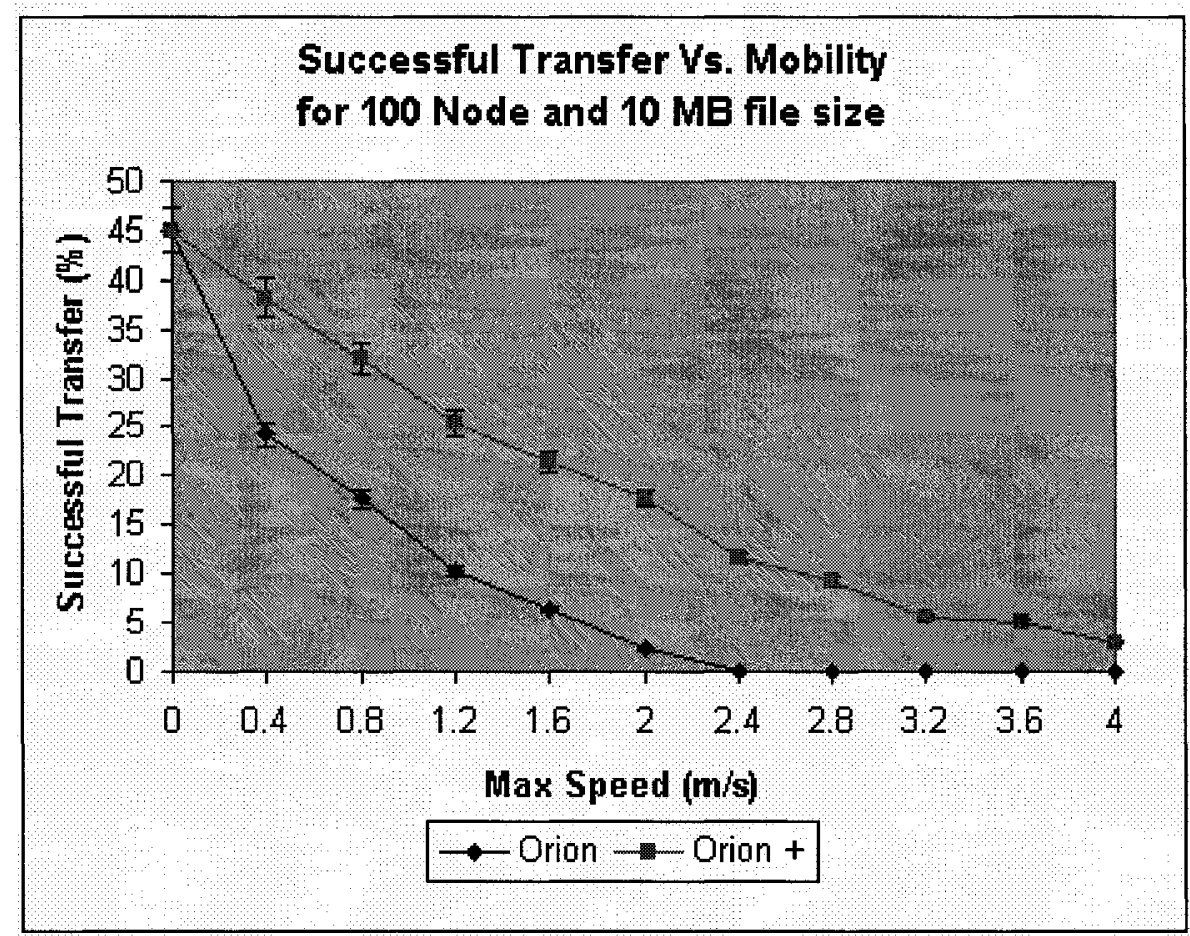

Figure 6.30 Successful Transfer vs. Node Mobility for 100 nodes and $10 \mathrm{MB}$ file size (Results are displayed with their $95 \% \mathrm{CI}$ ) 


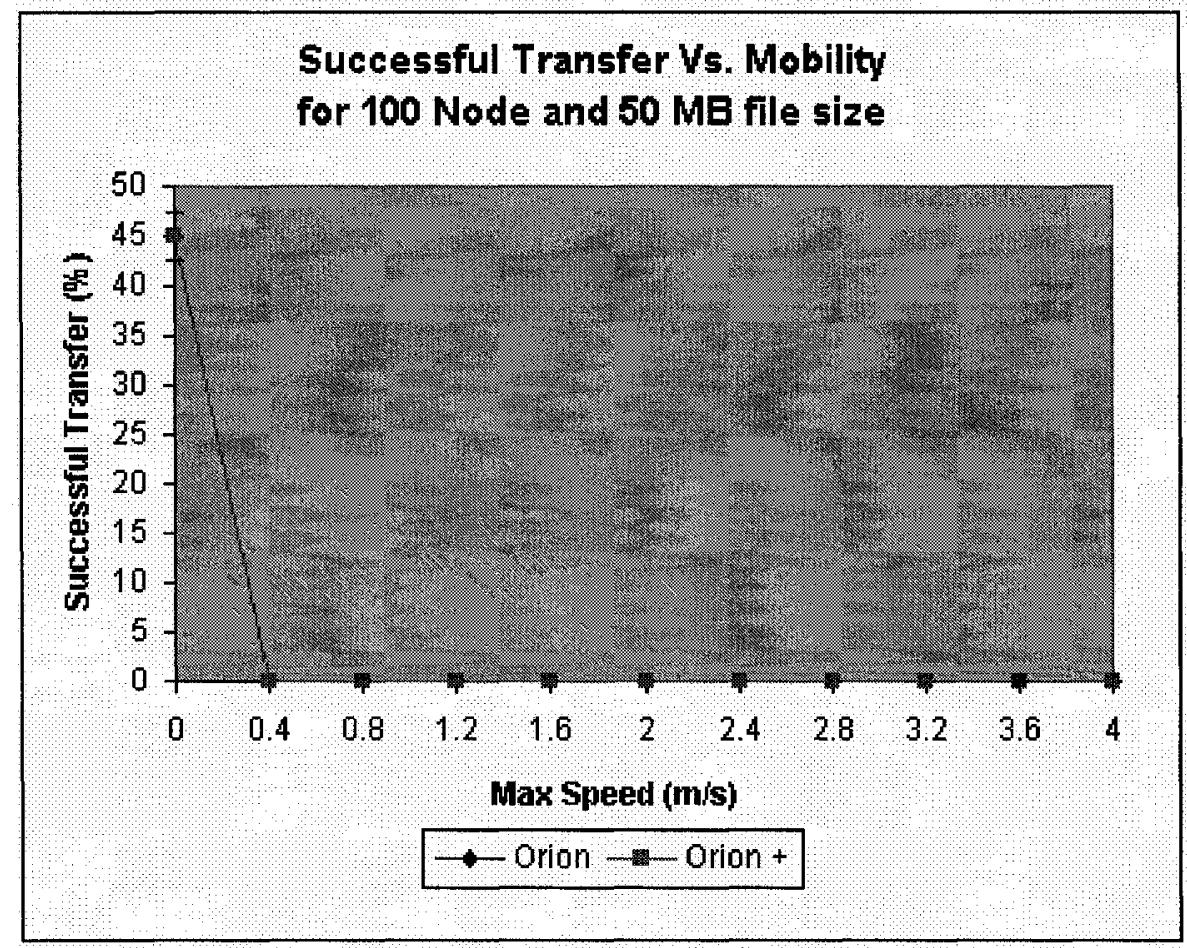

Figure 6.31 Successful Transfer vs. Node Mobility for 100 nodes and $50 \mathrm{MB}$ file size (Results are displayed with their $95 \%$ CI)

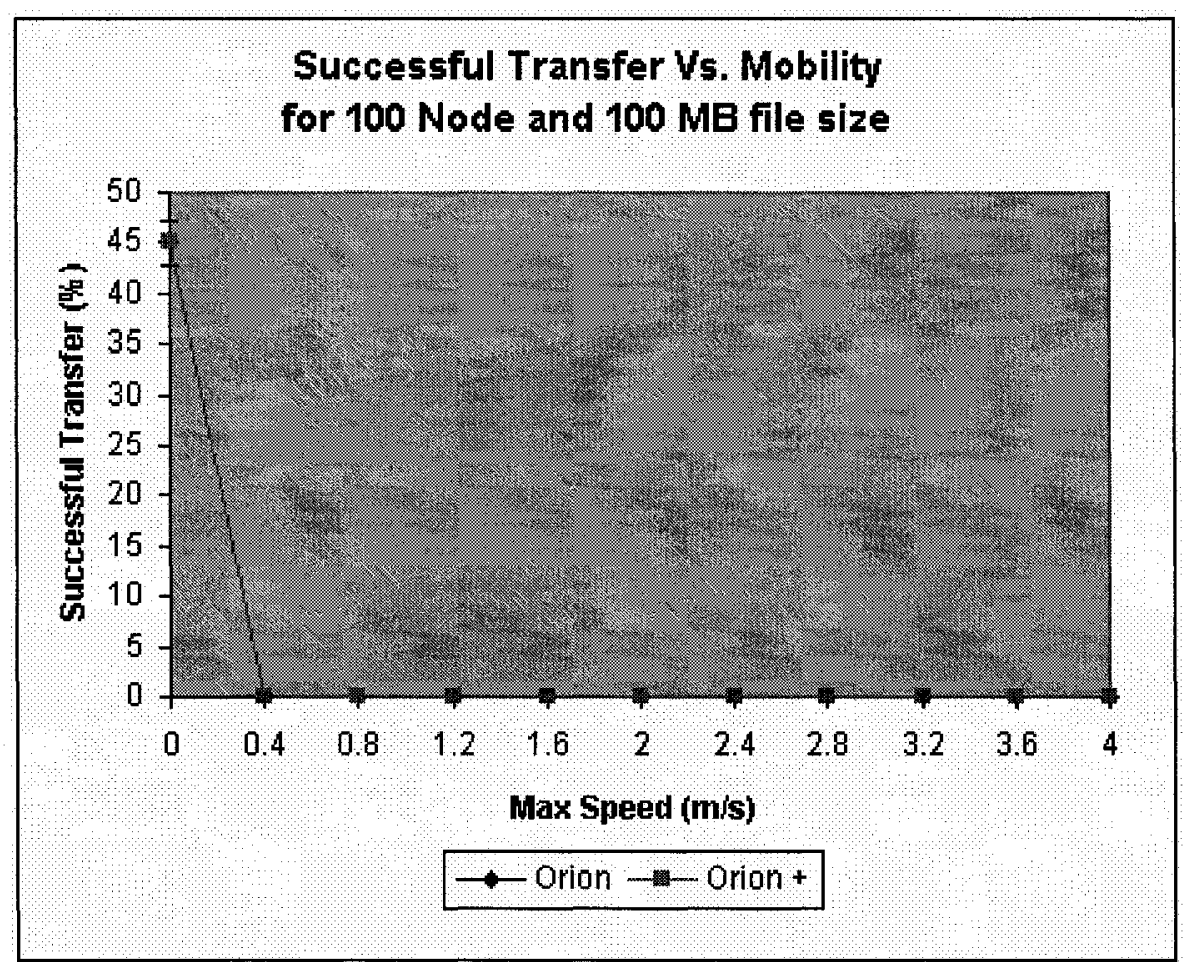

Figure 6.32 Successful Transfer vs. Node Mobility for 100 nodes and $100 \mathrm{MB}$ file size (Results are displayed with their $95 \%$ CI) 


\subsubsection{Transmitted Data Volume}

Due to the scarcity of available bandwidth in MANETs, it is important that any new feature we add to the ORION protocol comes at a reasonable price. It is hence very desirable to maintain our control traffic at a minimum so that the majority of the available network bandwidth can be used to transfer payload traffic. In this section we compare the total generated traffic injected in the network when using ORION and ORION+ under different file sizes and max speed scenarios. Figures 6.33 to 6.40 show the simulation results for the 50 nodes scenario and Figures 6.41 to 6.48 show the simulation results for the 100 nodes scenario for different file sizes. We compare the total transmitted data volume by measuring how many messages were generated in the network for each file transmitted from a source to a destination under each protocol. To do that, for each file transmitted from a source to a destination, we calculate how many data and control messages were generated in total for that file times the number of intermediate hops they went through. For example, if a certain message had to travel three hops to reach its destination, the total number of messages generated in the network is thrice as the original message.

With the actual file size being the absolute minimum for the transmitted data volume (assuming that the file is transmitted directly between the source and destination with no control overhead), we plotted our results to show the total transmitted data volume as a percentage of the actual file size. We can see here that ORION+ does not generate considerable traffic overhead more than the original ORION because its unicast messages are transmitted to a selected number of nodes and does not use flooding. The 
stable cost increase of ORION+ as the file sizes are increased leads to the conclusion that it is a scalable solution. Figures 6.3 and 6.34 show that because of the small file sizes (2 $\mathrm{KB}$ and $50 \mathrm{~KB}$ ) used in the low density scenario (50 nodes network), both of ORION and ORION+ generate the same number of messages because the files are always downloaded successfully before it is time to send out the ORION+ probing messages. Due to the usage of longer routes with more intermediate hops in the 100 node scenarios, we see that it have resulted in more transmitted messages than the 50 node scenarios.

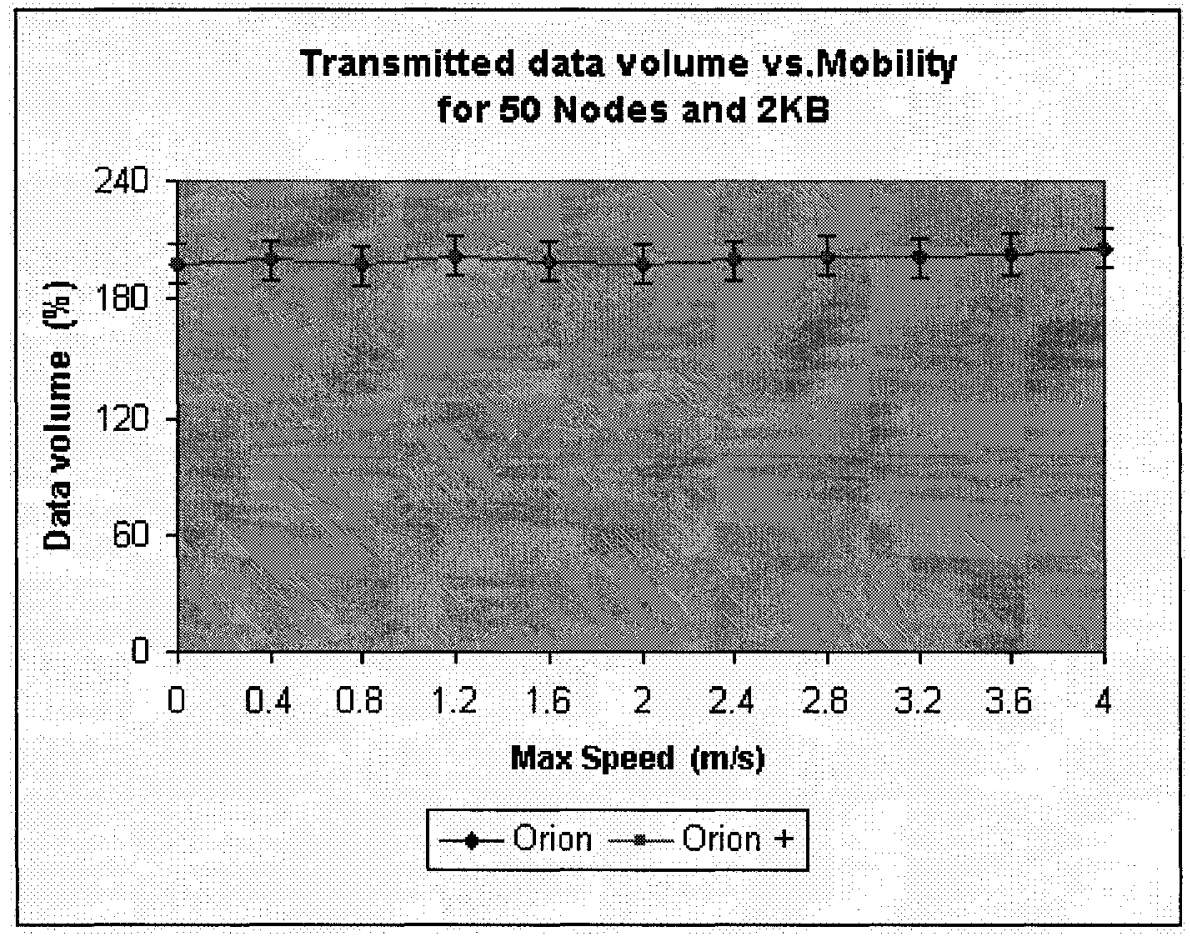

Figure 6.33 Data volume vs. Node Mobility for 50 nodes and 2 KB file size (Results are displayed with their $95 \%$ CI) 


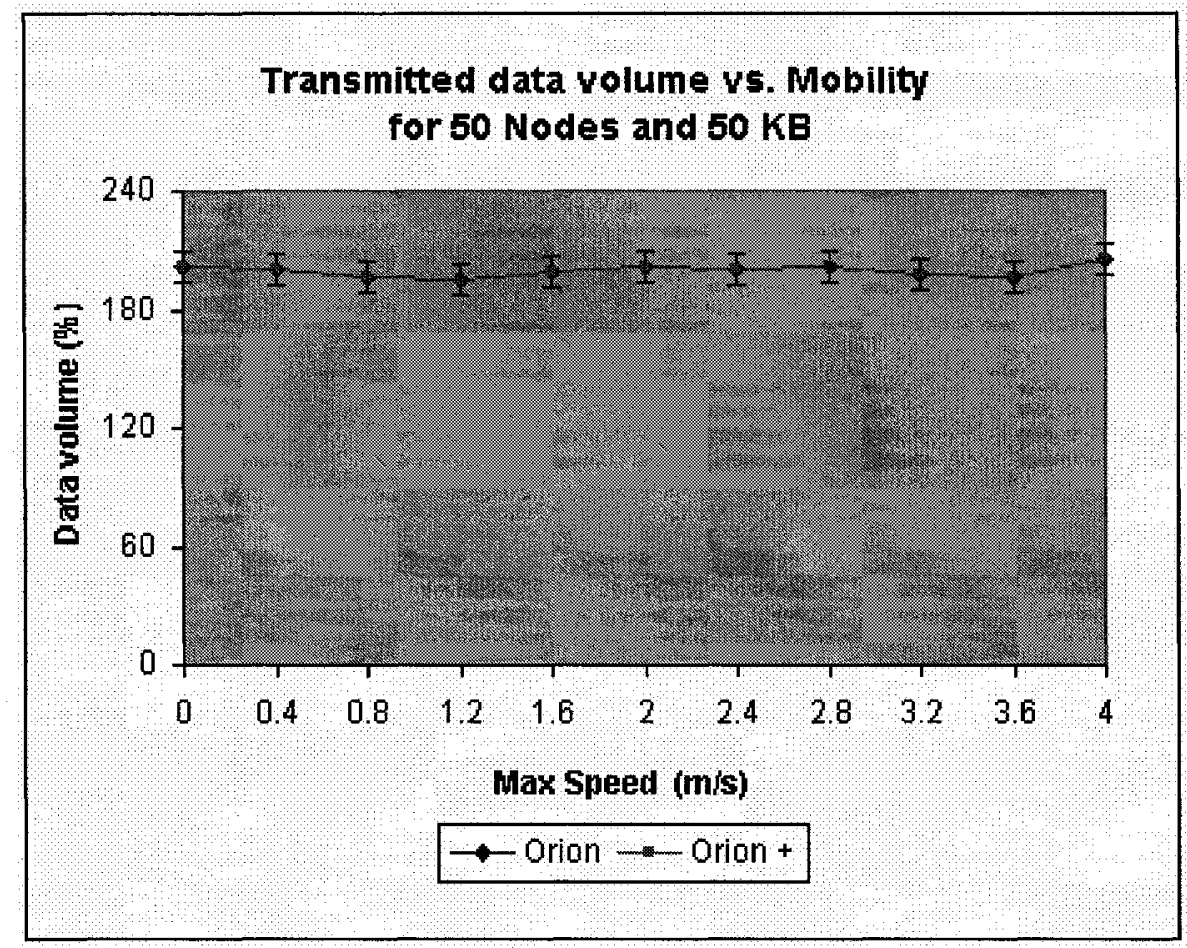

Figure 6.34 Data volume vs. Node Mobility for 50 nodes and 50 KB file size (Results are displayed with their $95 \% \mathrm{CI}$ )

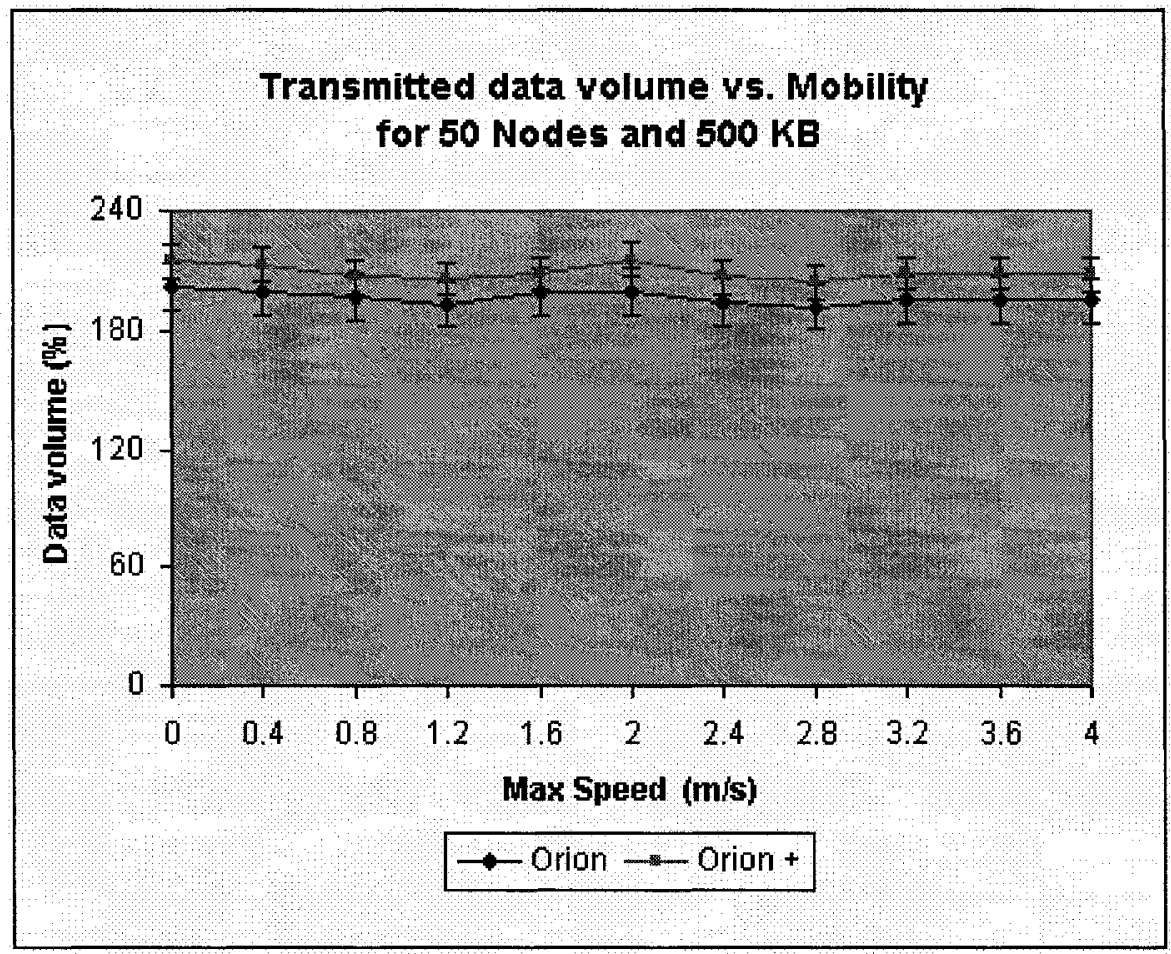

Figure 6.35 Data volume vs. Node Mobility for 50 nodes and $500 \mathrm{~KB}$ file size (Results are displayed with their $95 \% \mathrm{CI}$ ) 


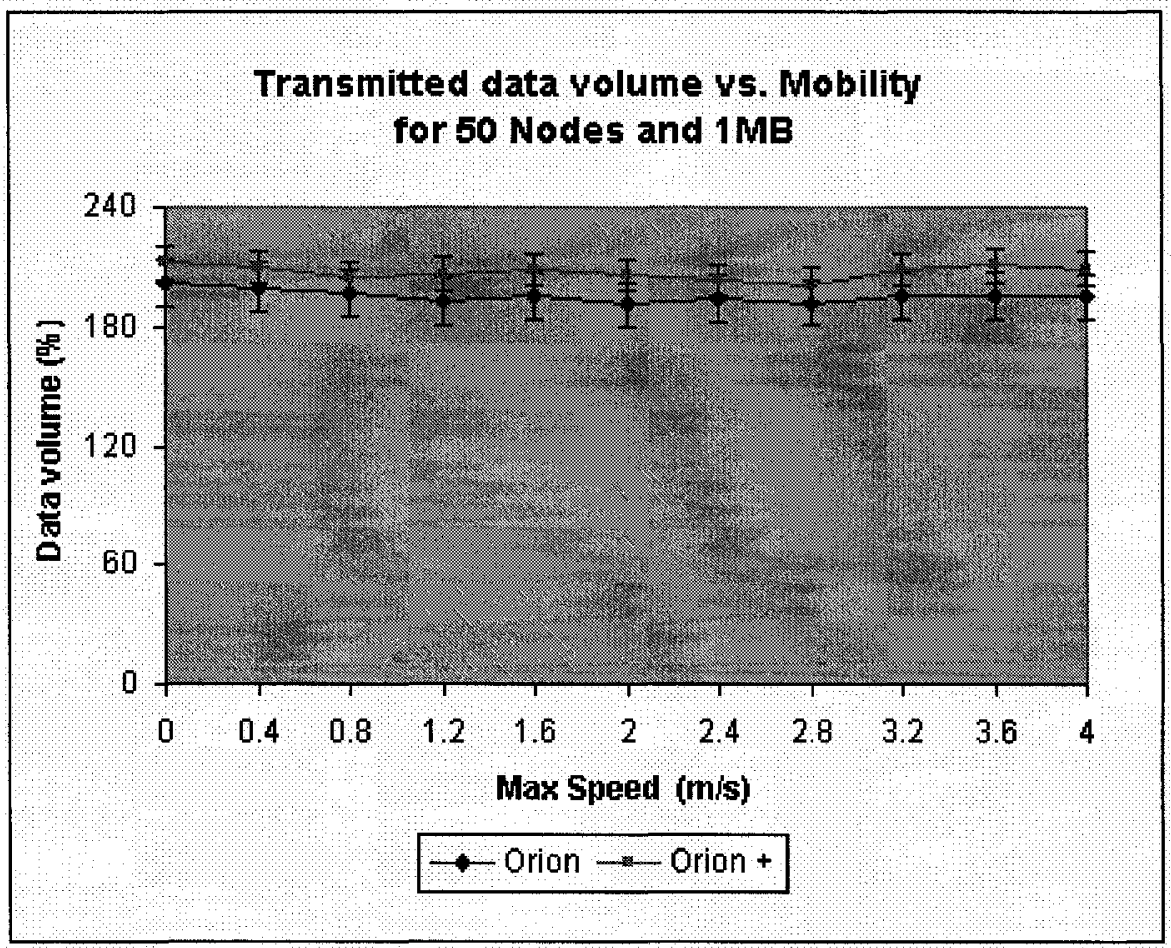

Figure 6.36 Data volume vs. Node Mobility for 50 nodes and 1 MB file size (Results are displayed with their $95 \% \mathrm{CI}$ )

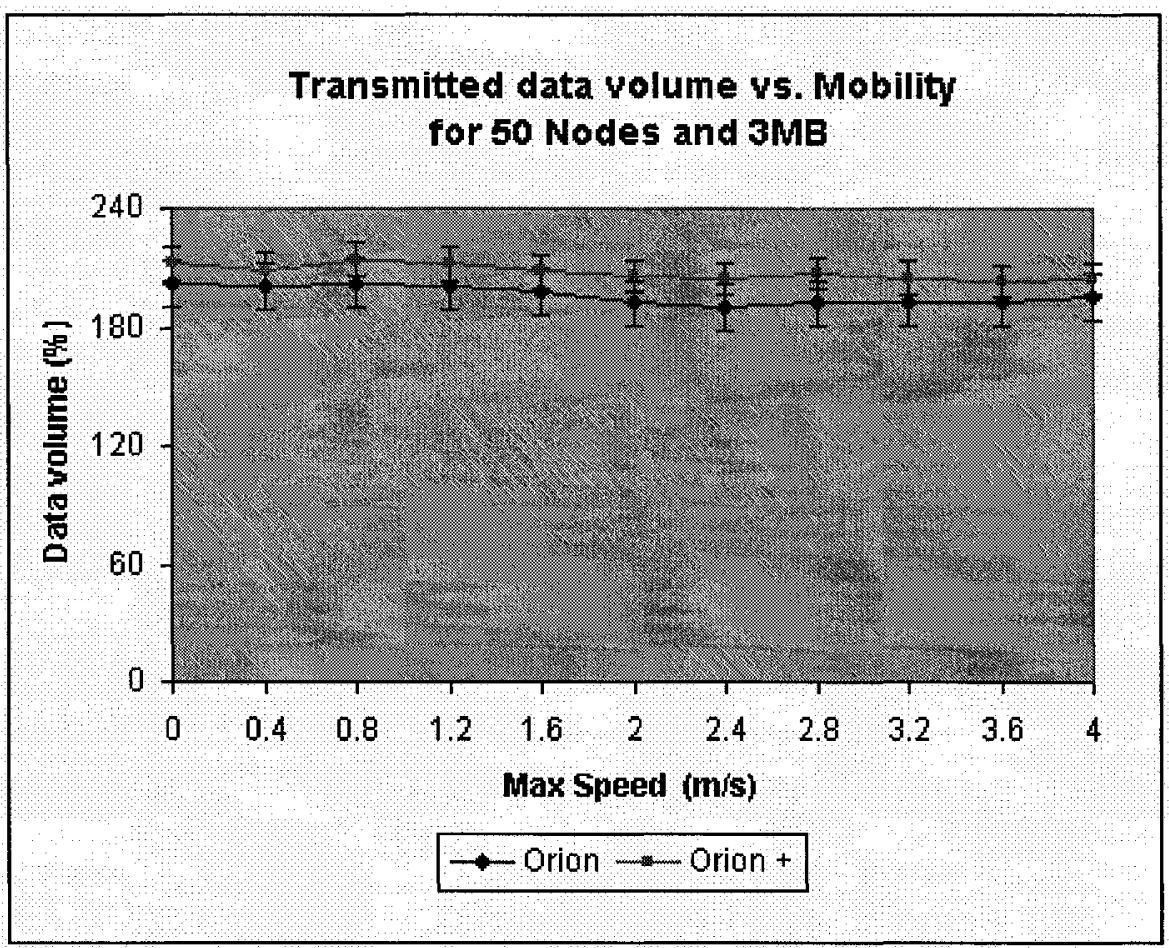

Figure 6.37 Data volume vs. Node Mobility for 50 nodes and $3 \mathrm{MB}$ file size (Results are displayed with their $95 \%$ CI) 


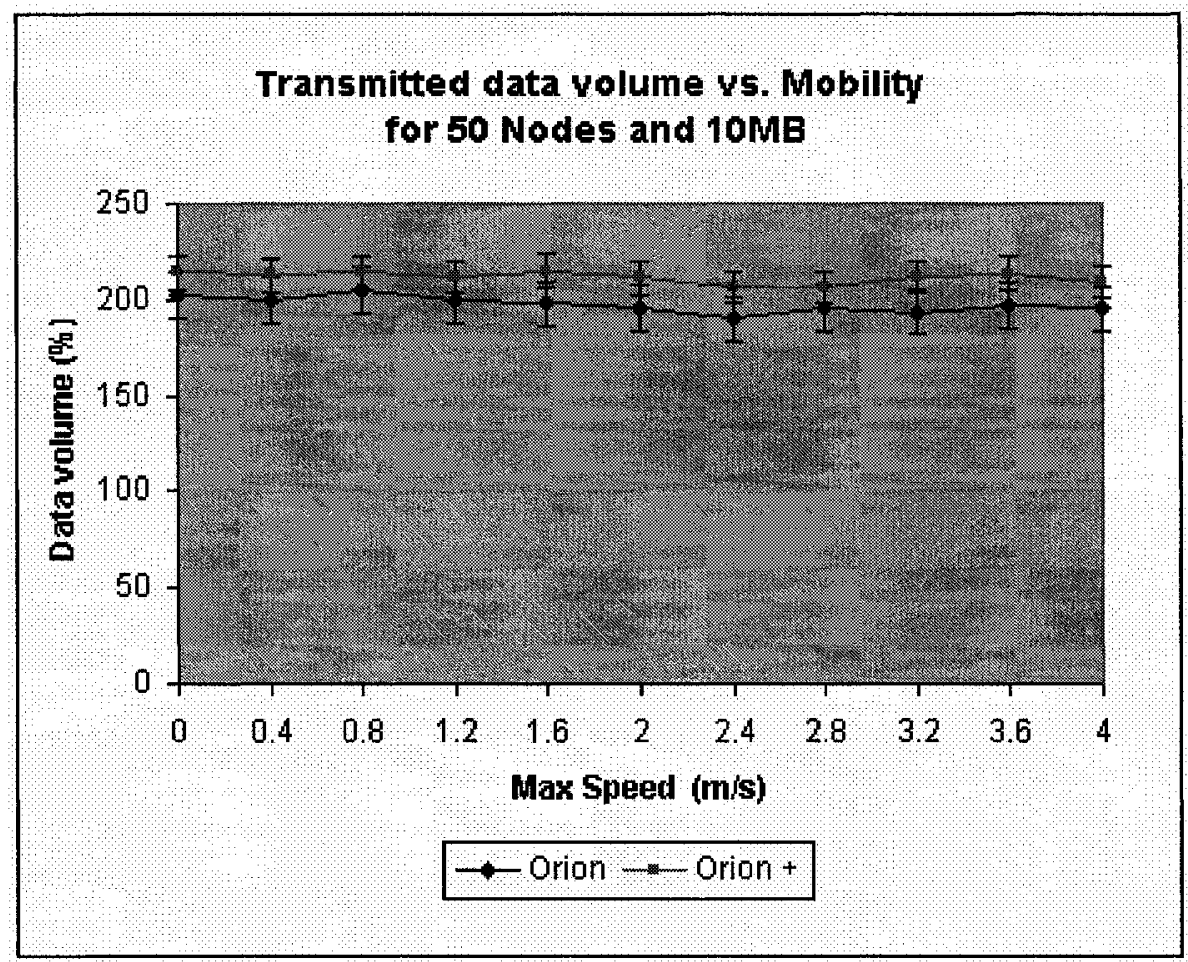

Figure 6.38 Data volume vs. Node Mobility for 50 nodes and $10 \mathrm{MB}$ file size (Results are displayed with their $95 \% \mathrm{CI}$ )

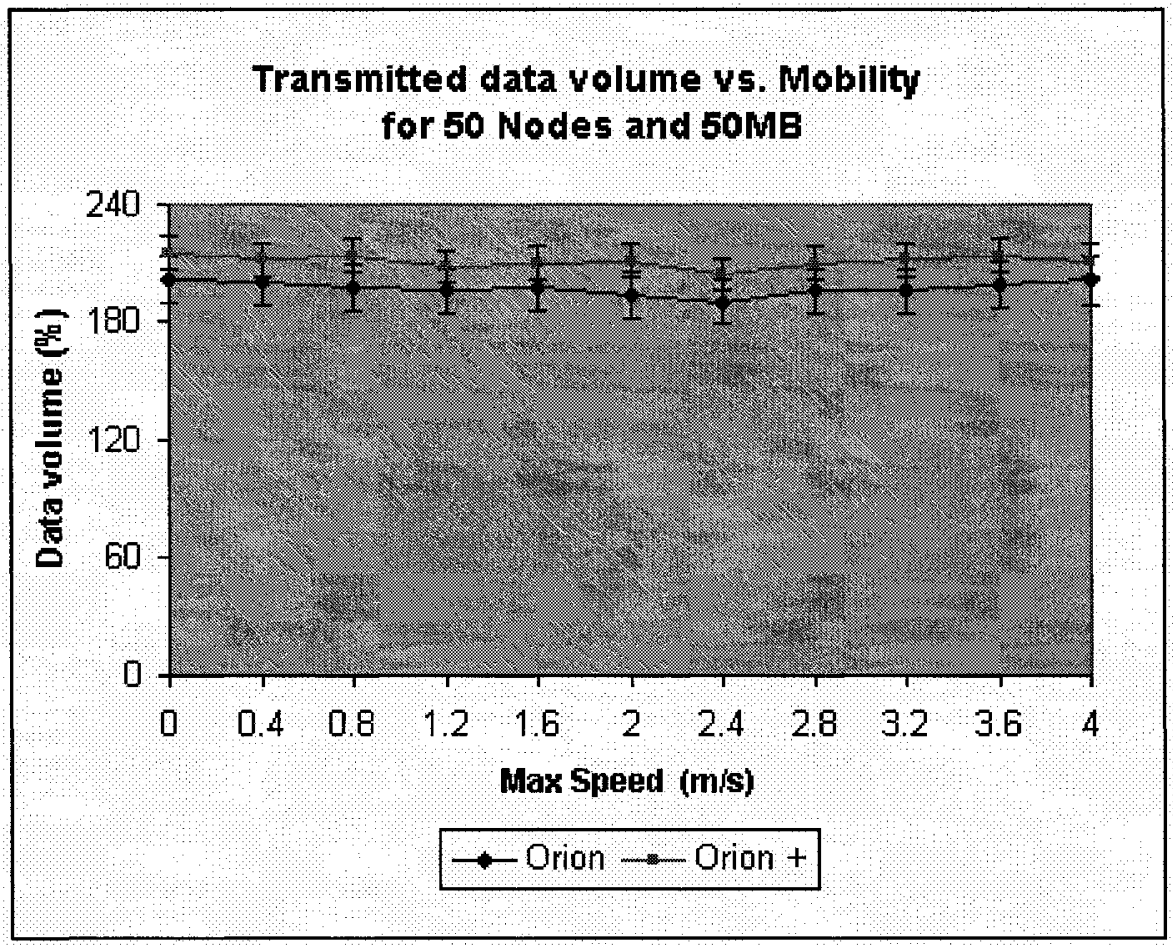

Figure 6.39 Data volume vs. Node Mobility for 50 nodes and $50 \mathrm{MB}$ file size (Results are displayed with their $95 \% \mathrm{CI}$ ) 


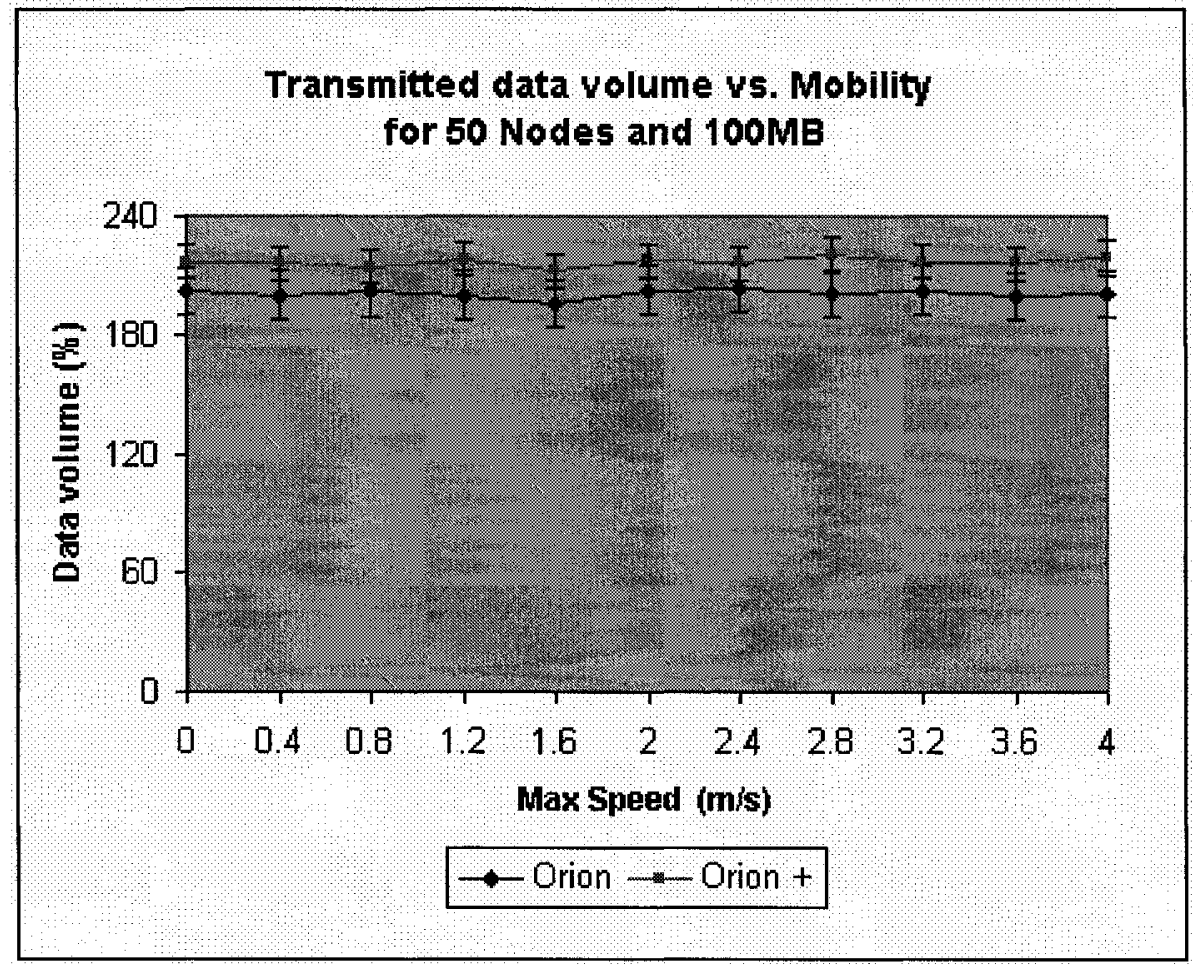

Figure 6.40 Data volume vs. Node Mobility for 50 nodes and $100 \mathrm{MB}$ file size (Results are displayed with their $95 \% \mathrm{CI}$ )

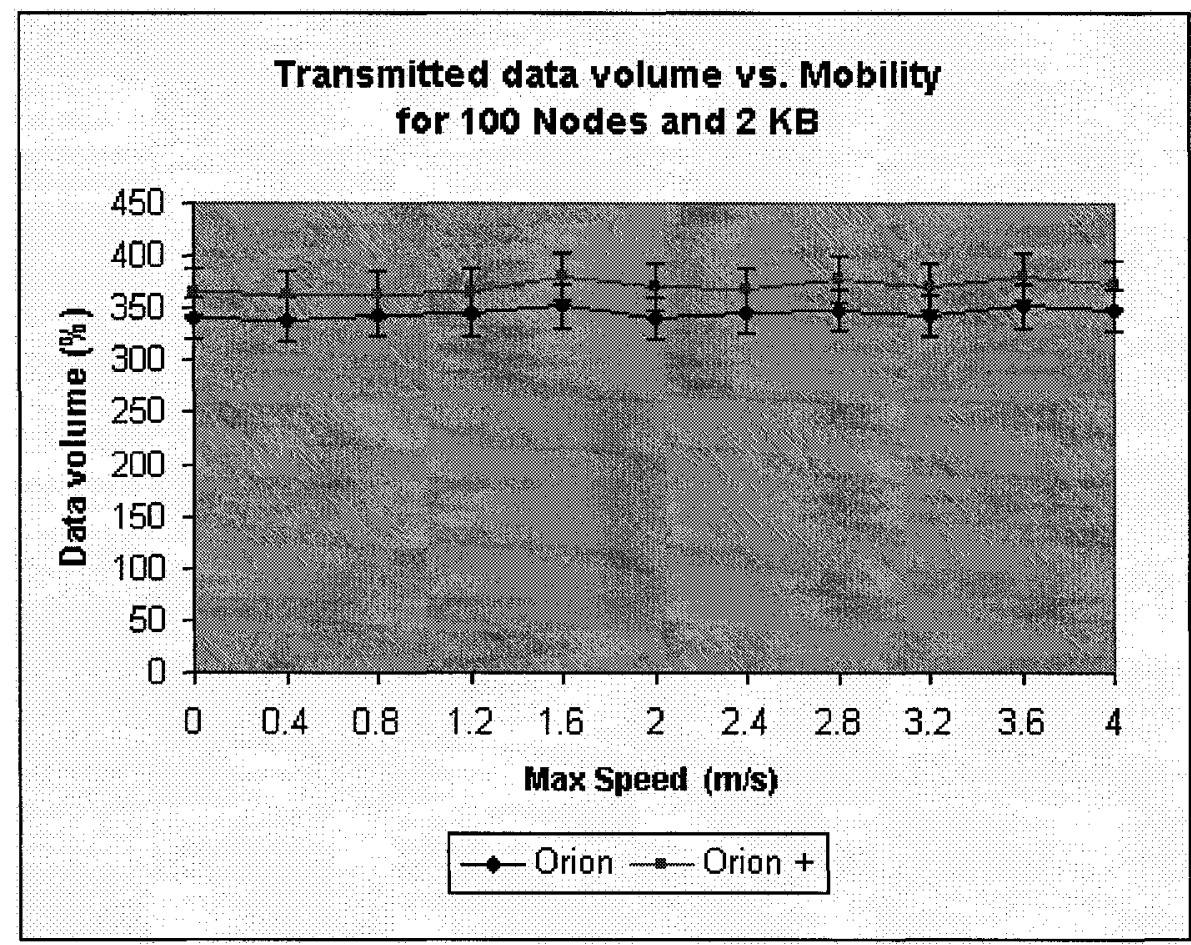

Figure 6.41 Data volume vs. Node Mobility for 100 nodes and $2 \mathrm{~KB}$ file size (Results are displayed with their $95 \%$ CI) 


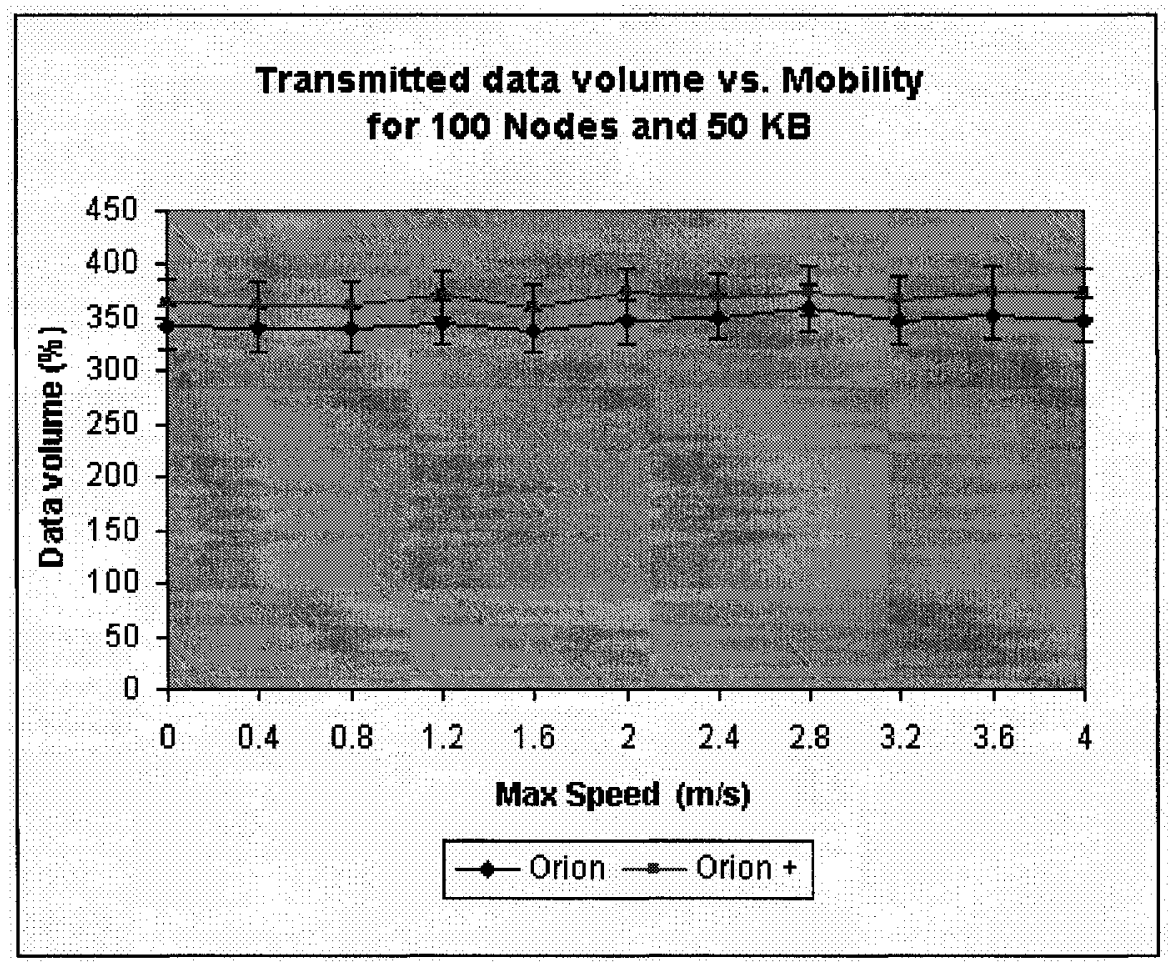

Figure 6.42 Data volume vs. Node Mobility for 100 nodes and $50 \mathrm{~KB}$ file size (Results are displayed with their $95 \% \mathrm{CI}$ )

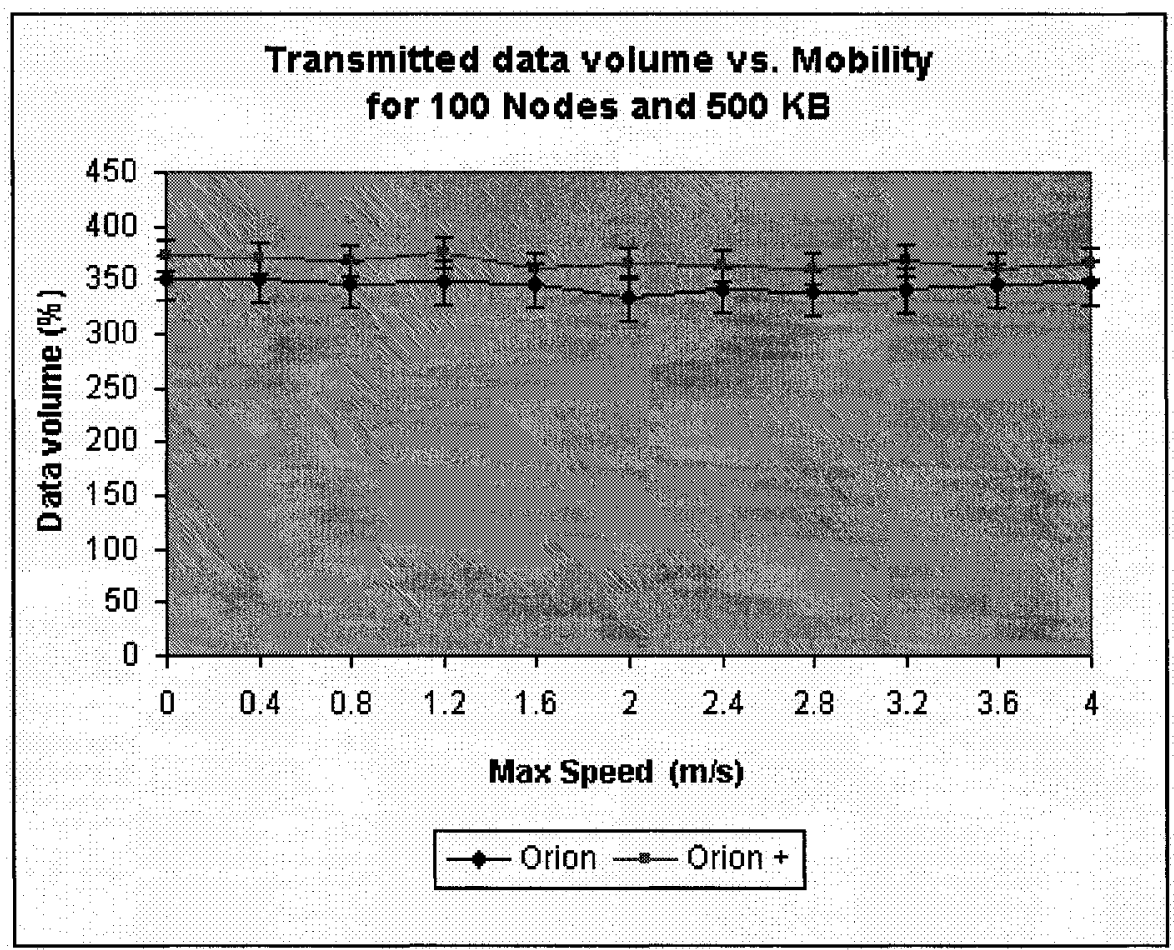

Figure 6.43 Data volume vs. Node Mobility for 100 nodes and $500 \mathrm{~KB}$ file size (Results are displayed with their $95 \%$ CI) 


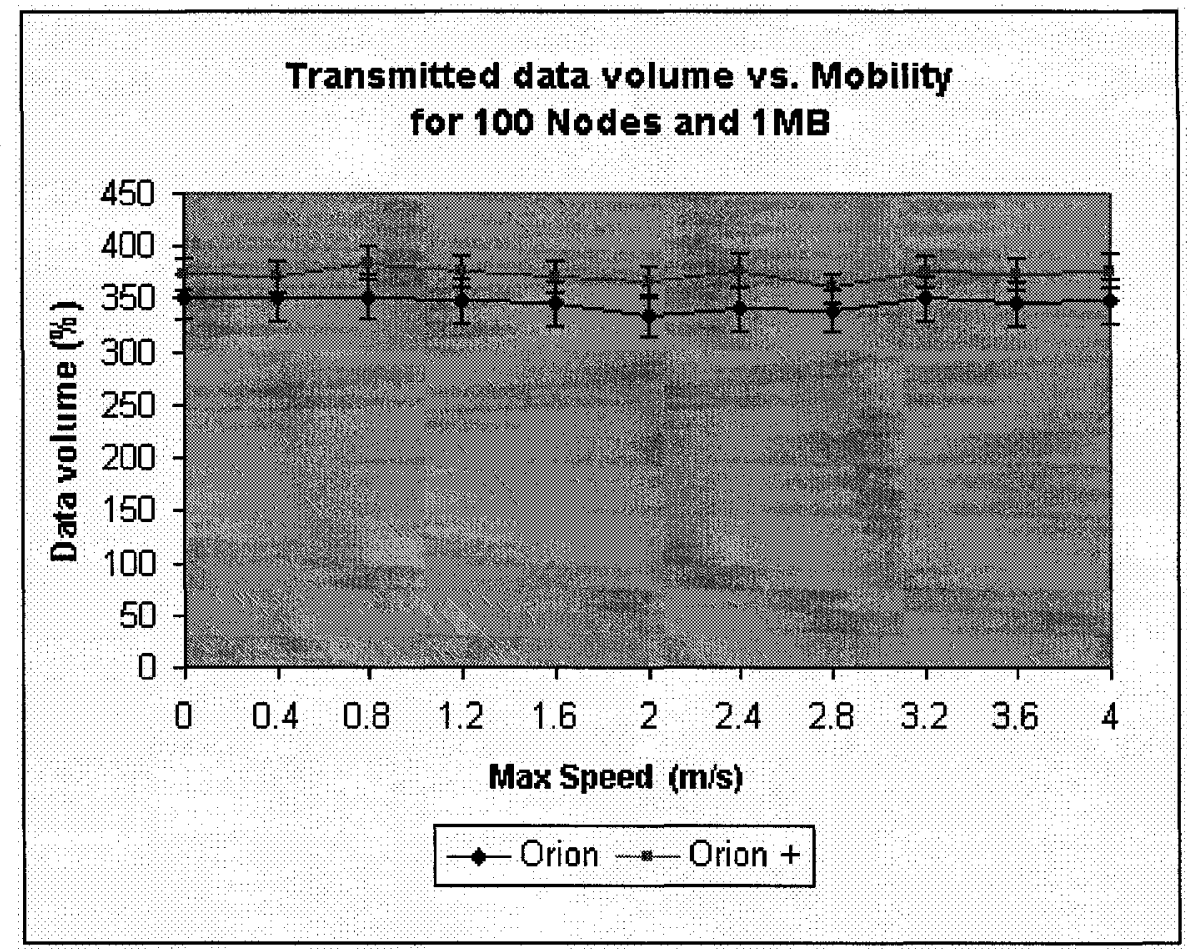

Figure 6.44 Data volume vs. Node Mobility for 100 nodes and $1 \mathrm{MB}$ file size (Results are displayed with their $95 \%$ CI)

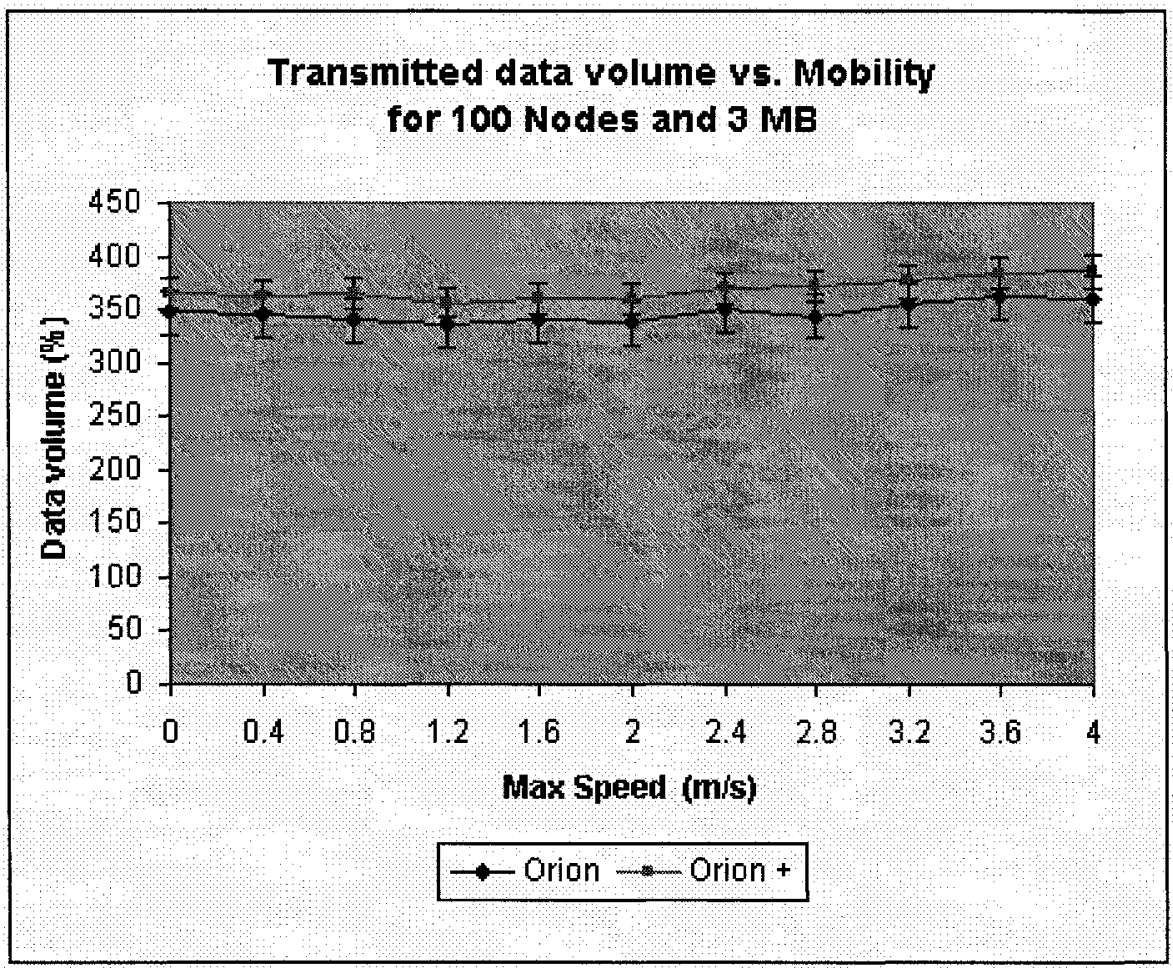

Figure 6.45 Data volume vs. Node Mobility for 100 nodes and $3 \mathrm{MB}$ file size (Results are displayed with their $95 \%$ CI) 


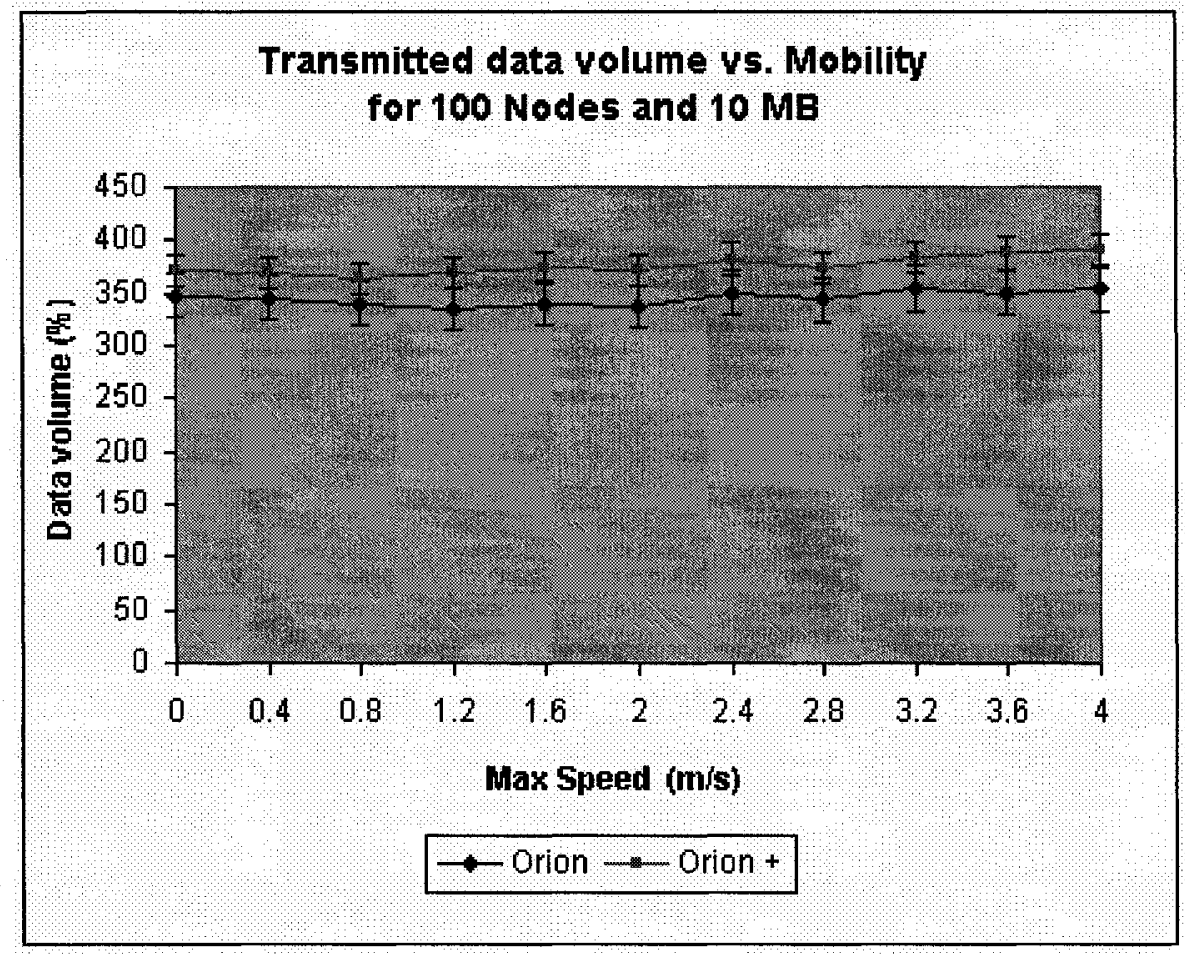

Figure 6.46 Data volume vs. Node Mobility for 100 nodes and 10 MB file size (Results are displayed with their $95 \% \mathrm{CI}$ )

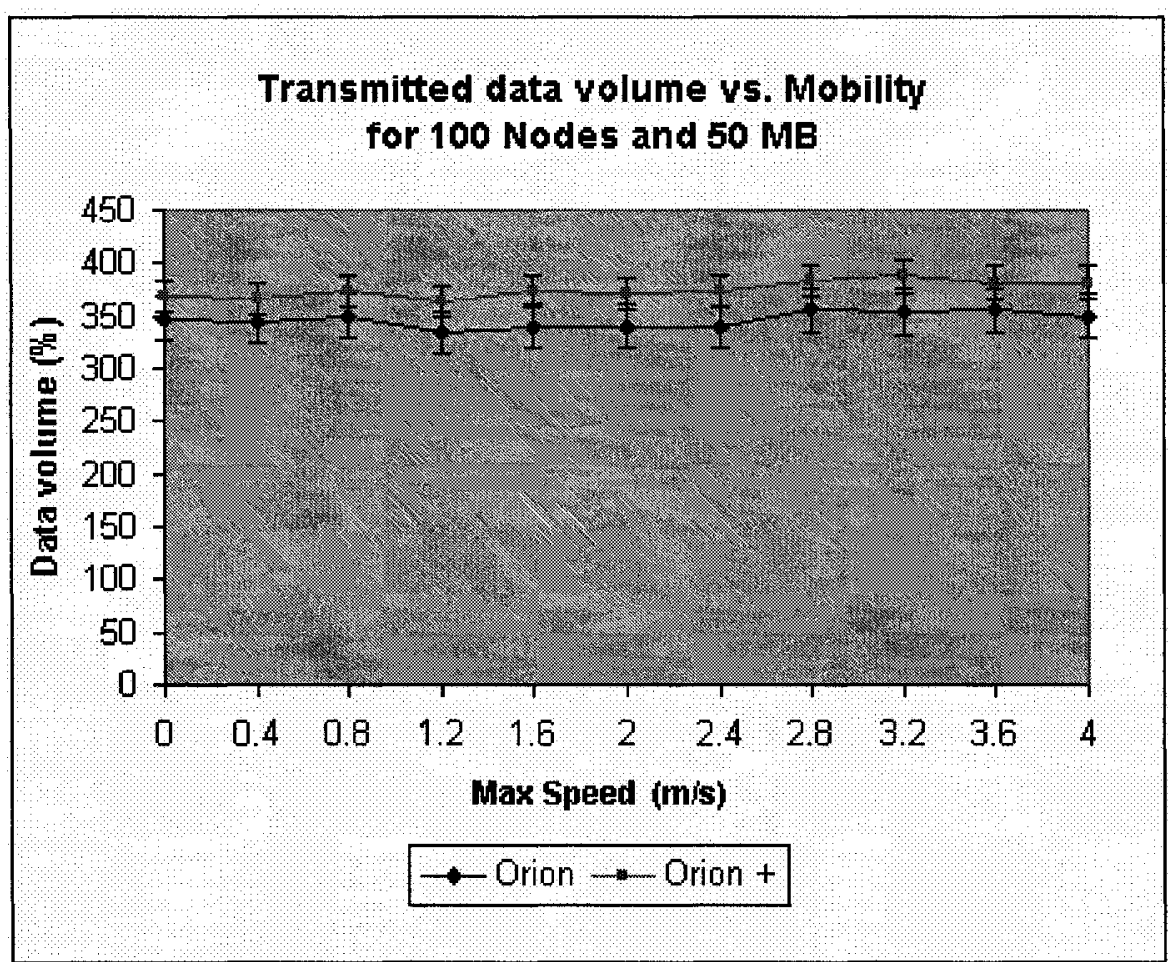

Figure 6.47 Data volume vs. Node Mobility for 100 nodes and 50 MB file size (Results are displayed with their $95 \% \mathrm{CI}$ ) 


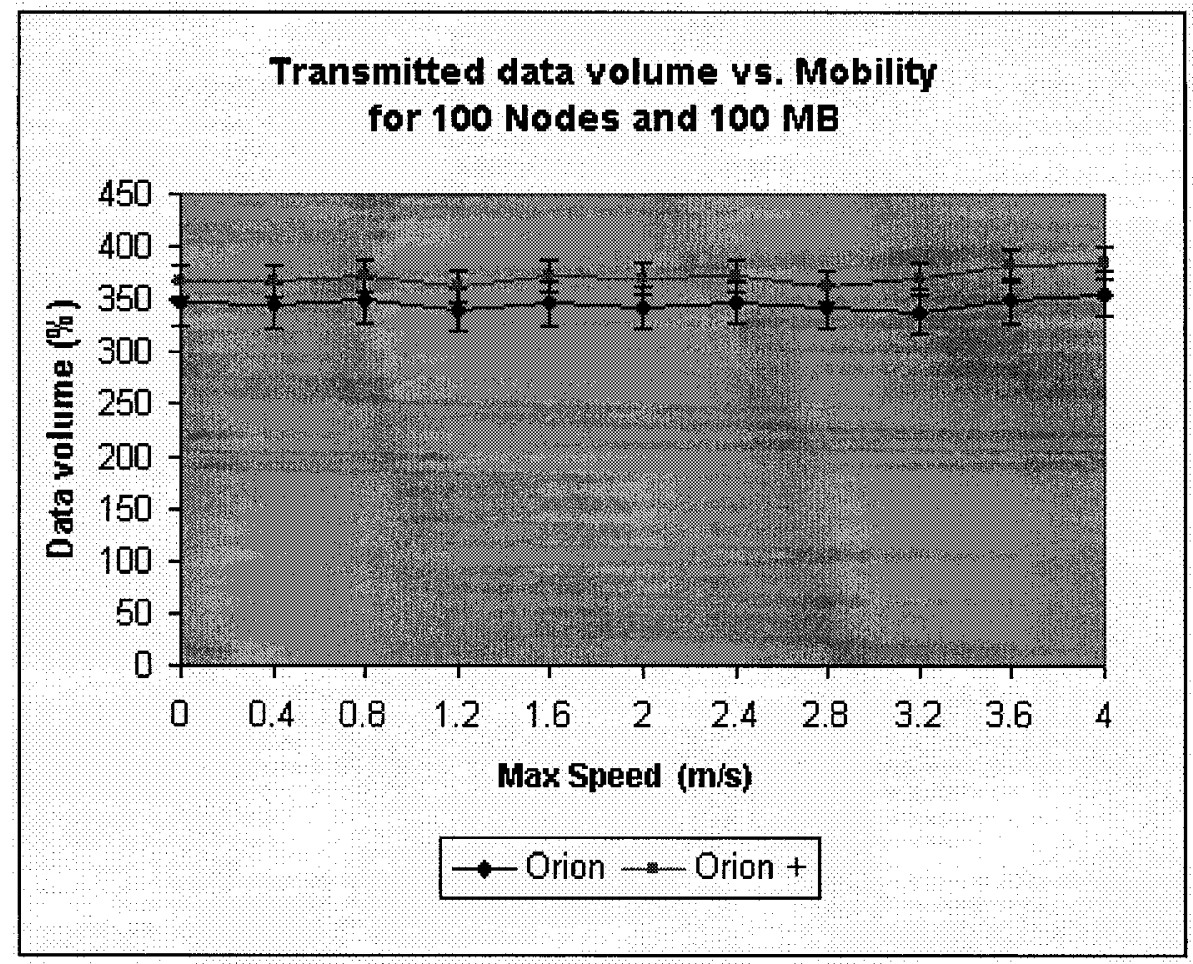

Figure 6.48 Data volume vs. Node Mobility for 100 nodes and $100 \mathrm{MB}$ file size (Results are displayed with their $95 \%$ CI) 


\section{Chapter 7 Conclusion and Future Work}

\subsection{Conclusion}

P2P file sharing applications are a very convenient way to provide content based routing in MANET environments where no central lookup server is available. However, many considerations have to be taken into account when combining the two together, mainly because of the inherit limitations of MANETs and the dynamic nature of P2P file sharing applications. Since path failures usually happen in MANETs P2P systems as a result of the dynamic physical topology, current P2P applications for MANETs have mainly used two path selection and recovery mechanisms (reactive and aggressive) to recover from path failures. Both of the reactive and the aggressive path selection and recovery mechanisms represent extreme measures to deal with path failures in MANETs P2P systems. While the aggressive mechanism seeks to always use the best path available in the network for file transfer, it usually have to initiate frequent network-wide flooding to search for a new path after each failure. On the other hand, the reactive mechanism requires less network-wide flooding because it usually has multiple alternative routes available for file transfer, but the down side of the reactive mechanism is that it would occasionally run into weakly connected paths and it does not make any effort to switch to a better connected path even if one existed already in its set of available paths.

In this thesis we presented a new path selection and recovery mechanism for MANETs P2P applications. It was based on the reactive path selection and recovery mechanism in order to avoid the frequent flooding of the aggressive mechanism. We 
called our new path selection and recovery mechanism "Reactive with unicast probing messages" and used it as the main building block of our new extension of the ORION protocol that we called ORION+. Our new path selection and recovery mechanism uses unicast probing messages to measure the roundtrip time of each available path so the one with the smallest roundtrip time can be used for the actual file transfer. Our path selection and recovery mechanism have helped to achieve lower file transfer times, higher percentage of successful file transfers and due to the unicast nature of its added control messages, it was proven to be scalable as per our simulation results.

\subsection{Future Work}

The new path selection and recovery mechanism (reactive with unicast probing messages) of ORION+ is able to select the best available path for file transfer based on the roundtrip time metric. But when all remaining paths are weakly connected, the system will have to wait for all of them to break (during that time it will be using the best available path) before it can initiate a new file search to get another set of fresh paths. A good idea for future research would be to establish a threshold level of acceptable roundtrip time, so that a new file search (flooding) would be required if no remaining paths are able to provide a roundtrip time lower than that of the threshold level. By doing so, we will further avoid using paths with longer roundtrip time and only initiate file search flooding when needed based on the acceptable threshold. Of course the question that needs to be solved here is how to calculate such a threshold in a totally random environment like MANETs. 


\section{References}

[1] A. Klemm, C. Lindemann, and O. Waldhorst, "A Special-Purpose Peer-toPeer File Sharing System for Mobile Ad Hoc Networks", Proc. IEEE Semiannual Vehicular Technology Conference (VTC 2003-Fall), Orlando, FL, October 2003.

[2] David B. Johnson and David A. Maltz, "Dynamic Source Routing in Ad Hoc Wireless Networks", In Mobile Computing, edited by Tomasz Imielinski and Hank Korth, Chapter 5, pages 153-181, Kluwer Academic Publishers, 1996.

[3] Ingo Gruber, Rüdiger Schollmeier and Wolfgang Kellerer, "Performance Evaluation of the Mobile Peer-to-Peer Protocol", In Proceedings of Fourth International Workshop on Global and Peer-to-Peer Computing, April 19 - 22, 2004, Chicago, USA.

[4] Charles E. Perkins and Elizabeth M. Royer, "Ad-hoc On-Demand Distance Vector Routing”, wmcsa, p. 90, Second IEEE Workshop on Mobile Computer Systems and Applications, 1999.

[5] S. Dhar, "MANET: Applications, Issues and Challenges for the Future", International Journal of Business Data Communications and Networking, Vol. 1, No. 2, April - June 2005, Pages 66-92. 
[6] E.M. Royer and C-K Toh, “A Review of Current Routing Protocols for Ad-Hoc Mobile Wireless Networks", IEEE Personal Communications, Apr. 1999

[7] Nikola Milanovic, Miroslaw Malek, Anthony Davidson and Veljko Milutinovic, "Routing and Security in Mobile Ad Hoc Networks", Computer, vol. 37, no. 2, pp. 61-65, February, 2004

[8] Jie Wu and Ivan Stojmenovic, "Ad Hoc Networks", IEEE Computer, February 2004

[9] R. Schollmeier, I. Gruber, and F. Niethammer, "Protocol for Peer-to-Peer Networking in Mobile Environments", In Proc. of 12th IEEE International Conference on Computer Communications and Networks, Dallas, Texas, USA, October 2003.

[10] David Remondo, "Tutorial on Wireless Ad Hoc Networks", HET-NET 2004: Second International Working Conference in Performance Modelling and Evaluation of Heterogeneous Networks, Ilkley, West Yorkshire, U.K., 26-28 ${ }^{\text {th }}$ July, 2004

[11] Ismail Ari, Neelu Jethani, Aniruddha Rangnekar and Sushma Natarajan, "Performance Analysis and Comparison of Ah-Hoc Routing Protocols", CMSC691T Mobile Computing Project Report, May 2000 
[12] Xiaoyan Hong, Kaixin Xu, and Mario Gerla, "Scalable Routing Protocols for Mobile Ad Hoc Networks", IEEE Network, special issue on Scalability in Communication Networks, July-Aug, 2002, pp. 11-21.

[13] Karl Aberer, Magdalena Punceva, Manfred Hauswirth and Roman Schmidt, "Improving Data Access in P2P Systems", IEEE Internet Computing, vol. 06, no. 1, pp. 58-67, January/February, 2002.

[14] J.A. Pouwelse, P. Garbacki, D.H. J. Epema, H. J. Sips, “The BitTorrent P2P FileSharing System: Measurements and Analysis", In $4^{\text {th }}$ International Workshop on Peer-to-Peer Systems (IPTPS'05), Feb 2005.

[15] B. Cohen, "Incentives build robustness in BitTorrent", In Proceedings of the First Workshop on the Economics of Peer-to-Peer Systems, Berkeley, CA, June 2003.

[16] Anna Hayes and David Wilson, "Peer-to-Peer Information Sharing in a Mobile Ad Hoc Environment”, wmcsa, pp. 154-162, Sixth IEEE Workshop on Mobile Computing Systems and Applications (WMCSA'04), 2004.

[17] Rüdiger Schollmeier, Ingo Gruber and Michael Finkenzeller, "Routing in Mobile Ad Hoc and Peer-to-Peer Networks. A Comparison", In Networking 2002, International Workshop on Peer-to-Peer Computing, Pisa, Italy, 19.-24.05.2002. 
[18] Baruch Awerbuch, David Holmer and Herbert Rubens, "Effects of Multi-rate in Ad Hoc Wireless Networks", technical report, www.cnds.jhu.edu/archipelago/.

[19] A. Kashyap, H. Nishar, and P. Agarwal, "Survey on unicast routing in mobile ad hoc networks", http://citeseer.ist.psu.edu/481660.html

[20] Gerd Kortuem, "Proem: A Middleware Platform for Mobile Peer-to-Peer Computing", ACM SIGMOBILE Mobile Computing and Communications Review (MC2R), Volume 6, Issue 4 (October 2002), Pages: 62 - 64.

[21] Leonardo B. Oliveira, Isabela G. Siqueira and Antonio A. F. Loureiro, "Evaluation of Ad hoc Routing Protocols under a Peer-to-Peer Application", IEEE Wireless Communications and Networking Conference, 2003 (WCNC'03). New Orleans, USA (pages 1143-1148)

[22] Milanovic N., Malek M., Davidson A. and Milutinovic V., "Routing and Security in Mobile Ad Hoc Networks", IEEE Computer, February 2004.

[23] Alex Varshavsky and Eyal de Lara, "Alleviating Self-Interference in MANETs", $4^{\text {th }}$ International IEEE Workshop on Wireless Local Networks (WLN 2004), Tampa, Florida 
[24] Ding Choon-Hoong, Sarana Nutanong, and Rajkumar Buyya, "Peer-to-Peer Networks for Content Sharing", Peer-to-Peer Computing: Evolution of a Disruptive Technology, Ramesh Subramanian and Brian Goodman (editors), ISBN: 1-59140-429-0, 28-65pp, Idea Group Publisher, Hershey, PA, USA, 2005

[25] Jörg Eberspächer, Rüdiger Schollmeier, Stefan Zöls and Gerald Kunzmann, "Structured P2P Networks in Mobile and Fixed Environments", In proceedings of HET-NETs '04 International Working Conference on Performance Modelling and Evaluation of Heterogeneous Networks, July 2004, Craiglands Hotel, Ilkley, West Yorkshire, U.K.

[26] Chonggang Wang and Bo Li, "Peer-to-peer overlay networks: A survey", Technical Report, Department of Computer Science, HKUST, Feb. 2003.

[27] Stephanos Androutsellis-Theotokis and Diomidis Spinellis, “A survey of peerto-peer content distribution technologies", ACM Computing Surveys, 36(4): 335-371, December 2004.

[28] I. Chlamtac, M. Conti and J. Liu, "Mobile Ad Hoc Networking: Imperatives and Challenges", Ad Hoc Networks, vol. 1, no. 1, 2003, pp. 13-64. 
[29] R. Schollmeier, I. Gruber, and M. Finkenzeller, "Routing in mobile ad hoc and peer-to-peer networks: a comparison", In Int.Workshop on Peer-to-Peer Computing, In Networking 2002, Pisa (Italy), May 2002.

[30] Stephanos Androutsellis-Theotokis and Diomidis Spinellis, "A survey of peerto-peer content distribution technologies", ACM Computing Surveys, 36(4):335-371, December 2004

[31] Y. Charlie Hu, Saumitra M. Das, and Himabindu Pucha, "Peer-to-Peer Overlay Abstractions in MANETs", In Theoretical and Algorithmic Aspects of Sensor, Ad Hoc Wireless and Peer-to-Peer Networks, edited by Jie Wu, CRC Press, 2004.

[32] Wolfgang Kellerer, Gerald Kunzmann, Rüdiger Schollmeier and Stefan Zöls, “Structured Peer-to-Peer Systems for Telecommunications and Mobile Environments", AEÜ - International Journal of Electronics and Communications January 2006,Volume: 0, Number: 0

[33] Carlos de M. Cordeiro and Dharma P. Agrawal, "Mobile Ad Hoc Networking", In 20th Brazilian Symposium on Computer Networks, Editors: Jose Rezende, Lucy Pirmez, and Luiz da Costa Carmo, ACS/NCE/UFRJ, CDD 004.6506 - pp. 125-186, May 2002. 
[34] Fox Harrell, Yuanfang Hu, Guilian Wang, and Huaxia Xia, "Survey of Locating \& Routing in Peer-to-Peer Systems", Final project for cse 221: Graduate operating systems, University of California, Sand Diego, Fall 2001. 